Japan. J. Math.

Vol. 31, No. 2, 2005

\title{
Real Hardy spaces on real rank 1 semisimple Lie groups
}

\author{
By Takeshi KawazoE* \\ (Received May 11, 2004) \\ (from Tokyo Journal of Mathematics)
}

\begin{abstract}
Let $G$ be a real rank one connected semisimple Lie group with finite center. We introduce a real Hardy space $H^{1}(G / / K)$ on $G$ as the space consisting of all $K$-bi-invariant functions $f$ on $G$ whose radial maximal functions $M_{\phi} f$ are integrable on $G$. We shall obtain a relation between $H^{1}(G / / K)$ and $H^{1}(\mathbf{R})$, the real Hardy space on the real line $\mathbf{R}$, via the Abel transform on $G$ and give a characterization of $H^{1}(G / / K)$.
\end{abstract}

\section{Introduction}

In the study of the classical Hardy spaces on the unit disk and the upper half plane, real variable characterizations of their boundary values are called the real variable method. In the 1970's these boundary values were completely characterized by various maximal functions without using the complex variable method and their atomic characterizations were also given at the same time. This was a significant breakthrough in harmonic analysis. Nowadays, this fruitful theory of real Hardy spaces, which are defined by maximal functions and atoms, has been extended to the spaces of homogeneous type: A topological space $X$ with measure $\mu$ and distance $d$ is of homogeneous type if there exists a constant $c>0$ such that for all $x \in X$ and $r>0$

$$
\mu(B(x, 2 r)) \leq c \mu(B(x, r)),
$$

where $B(x, r)$ is the ball defined by $\{y \in X \mid d(x, y)<r\}$ and $\mu(B(x, r))$ the volume of the ball (cf. $[2, \S 1])$.

When the space $X$ is not of homogeneous type, little work on real Hardy spaces on $X$ has been done. In this paper, looking at the example of a semisimple Lie group $G$ as a space of non-homogeneous type, we shall consider real Hardy spaces on $G$. Actually, when $X=G, \mu(B(x, r))$ has an exponential growth order as $r$ goes to infinity and hence $G$ is not of homogeneous type. We shall introduce a real Hardy space on $G$ by using a radial maximal function on $G$. Our goal is to give an

2000 Mathematics Subject Classification. Primary 22E30; Secondary 43A30, 43A80.

* Supported by Grant-in-Aid for Scientific Research (C), No. 13640190, Japan Society for the Promotion of Science. 
atomic characterization of the space and to obtain a relation with the real Hardy space $H^{1}(\mathbf{R})$ on $\mathbf{R}$.

We shall overview some results obtained in the previous papers [7], [8], [9] and state our main theorem. Let $G$ be a real rank one connected semisimple Lie group with finite center and $G=K A N=K C L\left(A^{+}\right) K$ respectively an Iwasawa and the Cartan decompositions of $G$. Let $d g=d k d a d n=\Delta(a) d k d a d k^{\prime}$ denote the corresponding decompositions of a Haar measure $d g$ on $G$. In this paper we shall treat only $K$-bi-invariant functions on $G$. Since $A$ is identified with $\mathbf{R}$ as $A=\left\{a_{x} ; x \in \mathbf{R}\right\}$, all $K$-bi-invariant functions can be identified with even functions on $\mathbf{R}$. We also denote $\Delta\left(a_{x}\right)$ by $\Delta(x)$ for $x \geq 0$ and extend it as an even function on $\mathbf{R}$. Then the one dimensional space $\mathbf{R}$ with normal distance and weighted measure $\Delta(x) d x$ is not of homogeneous type, because $\Delta(x) \sim e^{2 \rho|x|}$ as $|x| \rightarrow \infty$, where $\rho$ is a positive constant determined by a group structure (see (2), (4), (5)). Let $L^{p}(G / / K)$ denote the space of all $K$-bi-invariant functions on $G$ with finite $L^{p}$-norm:

$$
\|f\|_{p}=\left(\int_{0}^{\infty}|f(x)|^{p} \Delta(x) d x\right)^{1 / p}
$$

and $L_{\text {loc }}^{1}(G / / K)$ the space of all locally integrable, $K$-bi-invariant functions on $G$.

We shall introduce a real Hardy space on $G$ by using a radial maximal function. As in the Euclidean case, to define a radial maximal function we need to define a dilation $\phi_{t}, t>0$, of a function $\phi$ on $G$. Let $\phi$ be a positive compactly supported $C^{\infty}, K$-bi-invariant function on $G$ such that

$$
\int_{G} \phi(g) d g=\int_{0}^{\infty} \phi(x) \Delta(x) d x=1 .
$$

We define the dilation $\phi_{t}$ of $\phi$ as

$$
\phi_{t}(x)=\frac{1}{t} \frac{1}{\Delta(x)} \Delta\left(\frac{x}{t}\right) \phi\left(\frac{x}{t}\right) .
$$

Clearly, $\phi_{t}$ has the same $L^{1}$-norm as $\phi:\left\|\phi_{t}\right\|_{1}=\|\phi\|_{1}$ and, for $1 \leq p \leq \infty$, it gives an approximate identity in $L^{p}(G / / K)$ (see [3, Lemma 16]). Since this dilation has the same properties as in the Euclidean case, it is quite natural to introduce a radial maximal function $M_{\phi} f$ on $G$ as

$$
\left(M_{\phi} f\right)(g)=\sup _{0<t<\infty}\left|\left(f * \phi_{t}\right)(g)\right| \quad(g \in G) .
$$

As shown in [7, Theorem 3.4], this maximal operator $M_{\phi}$ satisfies the so-called maximal theorem: $M_{\phi}$ is bounded on $L^{p}(G / / K)(1<p \leq \infty)$ and satisfies the weak type $L^{1}$ estimate. Now we define the real Hardy space $H^{1}(G / / K)$ on $G$ 
analogously as in the real Hardy space $H^{1}(\mathbf{R})$ on $\mathbf{R}$ :

$$
H^{1}(G / / K)=\left\{f \in L_{\mathrm{loc}}^{1}(G / / K) ; M_{\phi} f \in L^{1}(G / / K)\right\}
$$

and the norm is given by

$$
\|f\|_{H^{1}(G)}=\left\|M_{\phi} f\right\|_{1} .
$$

The aim of this paper is to characterize $H^{1}(G / / K)$.

For a compactly supported $C^{\infty}, K$-bi-invariant function $f$ on $G$, we shall define the Abel transform $F_{f}^{s}, s \in \mathbf{R}$, of $f$ as

$$
F_{f}^{s}(x)=e^{\rho(1+s) x} \int_{N} f\left(a_{x} n\right) d n .
$$

This integral over $N$ is explicitly given by a composition of generalized Weyl type fractional integrals (see [11, Corollary 3.3] and (14)). Especially, we put

$$
W_{+}(f)=F_{f}^{1}
$$

and denote by $W_{-}$the inverse operator of $W_{+}$, which is given by a composition of Weyl type fractional derivatives (see (18), (19)). Let $\hat{f}$ denote the spherical Fourier transform of $f$ on $G$ and $F^{\sim}$ the Euclidean Fourier transform of $F$ on $\mathbf{R}$. Both $\hat{f}$ and $\left(F_{f}^{s}\right)^{\sim}$ are regarded as functions on the dual space $\mathrm{F}$ of the Lie algebra of $A$, which is identified with $\mathbf{R}$. Then they are extended to holomorphic functions on $F_{c}$, the complexification of $F$, of exponential type and

$$
\hat{f}(\lambda+i s \rho)=\left(F_{f}^{s}\right)^{\sim}(\lambda), \quad \lambda \in \mathrm{F}_{c}
$$

(cf. $[11,(3.17)]$ ). Let $C(\lambda)$ denote Harish-Chandra's C-function (see (9)) and $\mathrm{M}_{C_{\rho}}$ the Euclidean Fourier multiplier corresponding to $C_{\rho}(\lambda)=C(\lambda+i \rho)$ :

$$
\mathrm{M}_{C_{\rho}}(F)^{\sim}(\lambda)=C(\lambda+i \rho) F^{\sim}(\lambda) .
$$

We here define

$$
W_{-}\left(H^{1}(\mathbf{R})\right)=\left\{f \in L_{\mathrm{loc}}^{1}(G / / K) ; W_{+}(f) \in H^{1}(\mathbf{R})\right\}
$$

and also $W_{-}\left(\mathrm{M}_{C_{\rho}}\left(H^{1}(\mathbf{R})\right)\right)$ by replacing the condition that $W_{+}(f) \in H^{1}(\mathbf{R})$ in the above definition with $\mathbf{M}_{C_{\rho}}^{-1} \circ W_{+}(f) \in H^{1}(\mathbf{R})$.

THEOREM A. Let notations be as above.

$$
W_{-}\left(\mathrm{M}_{C_{\rho}}\left(H^{1}(\mathbf{R})\right)\right) \subset H^{1}(G / / K) \subset W_{-}\left(H^{1}(\mathbf{R})\right) .
$$

This is one of the main results in [8]. However, the proof was a little bit complicated, because to obtain the first inclusion we used the Harish-Chandra 
expansion of the zonal spherical function and also the Gangolli expansion (see (8), (10)). Thereby, to sum up the estimates of each expanded terms we required a sharp estimate and a deep theory of $H^{1}$ Fourier multipliers on $\mathbf{R}$. In this paper we shall give a simple proof based on fractional calculus on $G$. Actually, Theorem A follows from the next Theorem B: We define a maximal operator $M_{\phi}^{\mathbf{R}}$ and a fractional operator $W_{\gamma}^{\mathbf{R}}$ on $\mathbf{R}$ by Definition 3.2 and (30) respectively, and $\underline{n} \in \mathbf{N}$ and the set $\underline{D}$ by (37) and (38) respectively.

Theorem B. There exist $0<c_{1} \leq c_{2}$ such that for all $f \in H^{1}(G / / K)$

$$
c_{1}\|f\|_{H^{1}(G)} \leq \sum_{m=0}^{\underline{n}} \sum_{\xi \in \underline{D}}\left\|M_{\phi}^{\mathbf{R}} \circ W_{-(m+\xi)}^{\mathbf{R}}(F)(x)(\operatorname{th} x)^{m+\xi}\right\|_{L^{1}(\mathbf{R})} \leq c_{2}\|f\|_{H^{1}(G)},
$$

where $F=W_{+}(f)$.

We next introduce atomic Hardy spaces on $G$. In the Euclidean space the atomic Hardy space $H_{\infty, 0}^{1,}(\mathbf{R})$ coincides with $H^{1}(\mathbf{R})$ (cf. [6, Theorem 3.30], [15, §2 in Chap. 3]). However, it may not be true in our setting, because the Lebesgue measure $d x$ is replaced by the weighted measure $\Delta(x) d x$. We denote the interval $\left[x_{0}-r, x_{0}+r\right]$ by $R\left(x_{0}, r\right)$ and set the volume by

$$
\left|R\left(x_{0}, r\right)\right|=\int_{x_{0}-r}^{x_{0}+r} \Delta(x) d x .
$$

We say that a $K$-bi-invariant function $a$ on $G$ is a $(1, \infty, 0)$-atom on $G$ provided that there exist $x_{0} \geq 0$ and $r>0$ such that

$$
\begin{gathered}
\operatorname{supp}(a) \subset R\left(x_{0}, r\right), \\
\|a\|_{\infty} \leq\left|R\left(x_{0}, r\right)\right|^{-1} \\
\int_{0}^{\infty} a(x) \Delta(x) d x=0 .
\end{gathered}
$$

Here $a$ is identified with a function on $\mathbf{R}_{+}$. Then $H_{\infty, 0}^{1}(G / / K)$ is defined by

$$
H_{\infty, 0}^{1}(G / / K)=\left\{f=\sum_{i} \lambda_{i} a_{i} ; a_{i} \text { is a }(1, \infty, 0) \text {-atom on } G \text { and } \sum_{i}\left|\lambda_{i}\right|<\infty\right\}
$$

and the norm is given by

$$
\|f\|_{H_{\infty, 0}^{1}(G)}=\inf \sum_{i}\left|\lambda_{i}\right|
$$

where the infimum is taken over all such representations $f=\sum_{i} \lambda_{i} a_{i}$. Moreover, we define $H_{\infty, 0}^{1, \epsilon}(G / / K)(\epsilon \geq 0)$ and $H_{\infty, 0}^{1,+}(G / / K)$ by replacing (ii) and (iii) of the 
above definition of $(1, \infty, 0)$-atom $a$ on $G$, respectively, with

$$
(i i)_{\epsilon} \quad\|a\|_{\infty} \leq\left|R\left(x_{0}, r\right)\right|^{-1}(1+r)^{-\epsilon}
$$

and .

$$
(i i i)_{+} \quad \int_{0}^{\infty} a(x) \Delta(x) d x=0 \quad \text { if } r \leq 1 .
$$

Clearly, for $\epsilon \geq 0$,

$$
H_{\infty, 0}^{1, \epsilon}(G / / K) \subset H_{\infty, 0}^{1}(G / / K) \subset H_{\infty, 0}^{1,+}(G / / K)
$$

We define a truncated maximal operator $M_{\phi}^{\text {loc }}$ on $G$ as

$$
\left(M_{\phi}^{\mathrm{loc}} f\right)(g)=\sup _{0<t<1}\left|\left(f * \phi_{t}\right)(g)\right| \quad(g \in G) .
$$

In [9] we essentially proved that $M_{\phi}^{\text {loc }}$ is bounded from $H_{\infty, 0}^{1,+}(G / / K)$ to $L^{1}(G / / K)$. As for $M_{\phi}$, we shall prove that $M_{\phi}$ is bounded from $H_{\infty, 0}^{1,+}(G / / K) \cap W_{-}\left(H^{1}(\mathbf{R})\right)$ to $L^{1}(G / / K)$. This means that $H_{\infty, 0}^{1,+}(G / / K) \cap W_{-}\left(H^{1}(\mathbf{R})\right) \subset H^{1}(G / / K)$. Finally, as a refinement, we have the following main theorem.

Theorem C. Let notations be as above. Then

$$
H^{1}(G / / K)=H_{\infty, 0}^{1,+}(G / / K) \cap W_{-}\left(H^{1}(\mathbf{R})\right)
$$

Furthermore, $H_{\infty, 0}^{1, \epsilon}(G / / K) \cap W_{-}\left(H^{1}(\mathbf{R})\right), \epsilon \geq 0$, is dense in $W_{-}\left(H^{1}(\mathbf{R})\right)$ and especially, $H^{1}(G / / K)$ is dense in $W_{-}\left(H^{1}(\mathbf{R})\right)$.

This paper is organized as follows. We recall some basic notations in $\S 2$. Then we shall define a radial maximal function $M_{\phi} f$ on $G$ in $\S 3$ and obtain a key formula which reduces $M_{\phi} f$ to a Euclidean maximal function $M_{\phi}^{\mathbf{R}}\left(W_{+}(f)\right)$ on $\mathbf{R}$ (see Proposition 3.3). Theorem B follows from this formula and fractional calculus on $G$ and $\mathbf{R}$ in $\S 4$ (see Theorem 4.6). As an easy consequence of Theorem B, we can obtain a simple proof of Theorem A (see Remark 4.7), and moreover, a norm-equivalence between $\|f\|_{H^{1}(G)}$ and $\left\|M_{\phi}^{\text {loc }} f\right\|_{1}+\left\|W_{+}(f)\right\|_{H^{1}(\mathbf{R})}$ (see Theorem 4.9). In $\S 5$ we introduce atomic Hardy spaces on $G$. Then the inclusion $H_{\infty, 0}^{1,+}(G / / K) \cap W_{-}\left(H^{1}(\mathbf{R})\right)$ $\subset H^{1}(G / / K)$ follows from the estimates obtained in $\S 4$ and [9] (see Proposition 5.9). Next we shall consider a $(1, \infty,+)$-atomic decomposition of functions $f$ in $H^{1}(G / / K)$ (see Proposition 6.2). Then, combining with Theorem A, we can deduce our main Theorem $C$ (see Theorem 6.3 and Theorem 6.4). In $\S 7$ we shall consider $\left(H^{1}, L^{1}\right)$-boundedness of other operators on $G$; singular integrals, modified heat and the Poisson maximal operators (see Definition 7.2) and the Riesz transform on $G$ (see Definition 7.10). We summarize some calculations and make a 
comment on the dual space of $W_{-}\left(H^{1}(\mathbf{R})\right)$ in $\S 8$. In $\S 8$.A, we shall obtain a sharp estimate of $\Gamma_{m}(\lambda)$, meromorphic functions appearing in the Gangolli expansion of a spherical function (see (10)), and in $\S 8 . \mathrm{B}$, we shall obtain a norm-equivalence among some Euclidean maximal operators. In §8.C, we shall introduce a dual space of $W_{-}\left(H^{1}(\mathbf{R})\right)$ as a pull-back of $B M O(\mathbf{R})$ via the complex Fourier-Jacobi transform on $G$ (see (91) and Definition 8.7). In this paper we introduce many real Hardy spaces on $G$ as subspaces of $L^{1}(G / / K)$. We refer the reader to the figure in $\S 8 . \mathrm{D}$ for the understanding of main relationship among them.

\section{Notations}

Let $G$ be a real rank one connected semisimple Lie group with finite center and $G=K A N$ an Iwasawa decomposition of $G$. Let $\mathfrak{a}$ be the Lie algebra of $A$ and $\mathrm{F}=\mathfrak{a}^{*}$ the dual space of $\mathfrak{a}$. Let $\gamma$ be the positive simple root of $(G, A)$ determined by $N$ and $H$ the unique element in a satisfying $\gamma(H)=1$. Let $m_{1}$ and $m_{2}$ denote the multiplicities of $\gamma$ and $2 \gamma$ respectively. We put

$$
\alpha=\frac{m_{1}+m_{2}-1}{2}, \quad \beta=\frac{m_{2}-1}{2}, \quad \rho=\alpha+\beta+1 .
$$

We parameterize each element in $A$, $\mathfrak{a}$, and $\mathrm{F}$ as $a_{x}=\exp (x H), x H$, and $x \gamma(x \in \mathbf{R})$ respectively. In what follows we often identify these spaces $A, \mathfrak{a}, \mathrm{F}$ with $\mathbf{R}$ and their complexifications with $\mathbf{C}$. We put $A_{+}=\left\{a_{x} ; x \in \mathbf{R}_{+}\right\}$. Then, according to the Cartan decomposition $G=K C L\left(A_{+}\right) K$ of $G$, every $K$-bi-invariant functions $f$ on $G$ are determined by their restrictions to $C L\left(A_{+}\right)$and hence, they are identified with even functions on $\mathbf{R}$. We denote them by the same letter, that is, if $g \in K a_{\sigma(g)} K$ with $\sigma(g) \in \mathbf{R}_{+}$, then

$$
f(g)=f\left(a_{\sigma(g)}\right)=f(\sigma(g))=f(-\sigma(g)) .
$$

Let $d g$ denote the Haar measure on $G$. We denote by $L_{\text {loc }}^{1}(G / / K)$ and $L^{p}(G / / K)$, respectively, the spaces of locally integrable and $p$-th power integrable $K$-bi-invariant functions on $G$. Let $C_{c}^{\infty}(G / / K)$ denote the space of compactly supported $C^{\infty}$, $K$-bi-invariant functions on $G$. Then for $f \in L^{1}(G / / K)$ the Cartan decomposition of $G$ yields that

$$
\int_{G} f(g) d g=\int_{0}^{\infty} f(x) \Delta(x) d x
$$

where

$$
\Delta(x)=c(\operatorname{sh} x)^{2 \alpha+1}(\operatorname{sh} 2 x)^{2 \beta+1}, \quad x \geq 0 .
$$


We note that the order of $\Delta(x)$ is given by

$$
\Delta(x) \sim \begin{cases}x^{2 \alpha+1} & \text { if } 0 \leq x \leq 1 \\ e^{2 \rho x} & \text { if } 1<x<\infty\end{cases}
$$

where the symbol " " means that the ratio of the left side and the right side is bounded uniformly below and above by positive constants. We extend $\Delta(x)$ as an even function on $\mathbf{R}$. By using this weight function $\Delta(x)$ the $L^{p}$-norm $\|f\|_{p}$ of $f$ on $G$ can be rewritten as in (1). We denote the Euclidean $L^{p}$-norm on $\mathbf{R}$ by $\|\cdot\|_{L^{p}(\mathbf{R})}$.

Let $\Omega$ denote the Laplace-Beltrami operator on $G$ and $\varphi_{\lambda}(\lambda \in \mathrm{F})$ the normalized zonal spherical function on $G$, that is, the $K$-bi-invariant eigenfunction of $\Omega$ satisfying

$$
\Omega \varphi_{\lambda}=-\left(\lambda^{2}+\rho^{2}\right) \varphi_{\lambda} \text { and } \varphi_{\lambda}(0)=1 .
$$

By restricting $\varphi_{\lambda}$ to $A, \varphi_{\lambda}(x), x \in \mathbf{R}$, is a solution of

$$
\frac{d^{2}}{d x^{2}}+((2 \alpha+1) \operatorname{cth} x+(2 \beta+1) \operatorname{th} x) \frac{d}{d x}
$$

which satisfies $\varphi_{\lambda}(0)=1$ and $\varphi_{\lambda}^{\prime}(0)=0$. Hence, if $\alpha \notin-\mathbf{N}$, it is explicitly given by the Jacobi function of the first kind with order $(\alpha, \beta)$ :

$$
\varphi_{\lambda}(x)=F\left(\frac{\rho+i \lambda}{2}, \frac{\rho-i \lambda}{2} ; \alpha+1 ;-\operatorname{sh}^{2} x\right) .
$$

Clearly, $\varphi_{\lambda}(x)$ is even with respect to $\lambda, x$ and it is uniformly bounded on $x \in \mathbf{R}$ if $\lambda$ is in the tube domain

$$
\mathrm{F}(\rho)=\left\{\lambda \in \mathrm{F}_{c} ;|\Im \lambda| \leq \rho\right\}
$$

(cf. [3, Lemma 11]). For $\lambda \notin-i \mathbf{N}$, let $\Phi(\lambda, x)$ denote another solution, which is given by the Jacobi function of the second kind with order $(\alpha, \beta)$ :

$$
\Phi(\lambda, x)=\left(e^{x}-e^{-x}\right)^{i \lambda-\rho} F\left(\frac{\rho-2 \alpha-i \lambda}{2}, \frac{\rho-i \lambda}{2} ; 1-i \lambda ;-\operatorname{sh}^{-2} x\right) .
$$

Then for $\lambda \notin \mathbf{Z}, \varphi_{\lambda}(x)$ has the so-called Harish-Chandra expansion:

$$
\varphi_{\lambda}(x)=e^{-\rho x}\left(\Phi(\lambda, x) C(\lambda) e^{i \lambda x}+\Phi(-\lambda, x) C(-\lambda) e^{-i \lambda x}\right)
$$

where

$$
C(\lambda)=\frac{\Gamma(\alpha+1)}{2 \sqrt{\pi}} \frac{2^{\rho} \Gamma(i \lambda / 2) \Gamma((1+i \lambda) / 2)}{\Gamma((i \lambda+\rho) / 2) \Gamma((i \lambda+\rho-2 \beta) / 2)}
$$


We denote the Gangolli expansion of $\Phi(\lambda, x), x>0$, as

$$
\Phi(\lambda, x)=\sum_{m=0}^{\infty} \Gamma_{m}(\lambda) e^{-2 m x} .
$$

For some basic properties of $\varphi_{\lambda}(x), \Phi(\lambda, x)$, and the recursive definition of $\Gamma_{m}(\lambda)$ we refer to $[3, \S 2, \S 3]$ and $[17,9.1 .4,9.1 .5]$.

For $f \in L^{1}(G / / K)$ the spherical Fourier transform $\hat{f}(\lambda), \lambda \in \mathrm{F}$, of $f$ is defined by

$$
\hat{f}(\lambda)=\int_{G} f(g) \varphi_{\lambda}(g) d g
$$

Then $\hat{f}(\lambda)$ is even and continuously extended on $\mathrm{F}(\rho)$, which is holomorphic in the interior and

$$
|\hat{f}(\lambda)| \leq\|f\|_{1}, \quad \lambda \in \mathrm{F}(\rho) .
$$

For $f \in C_{c}^{\infty}(G / / K)$ the Paley-Wiener theorem (cf. [3, Theorem 4]) implies that $\hat{f}(\lambda)$ is holomorphic on $F_{c}$ of exponential type. Furthermore, it satisfies the inversion formula

$$
f(x)=\int_{-\infty}^{\infty} \hat{f}(\lambda) \varphi_{\lambda}(x)|C(\lambda)|^{-2} d \lambda
$$

and the Plancherel formula

$$
\int_{0}^{\infty}|f(x)|^{2} \Delta(x) d x=\int_{0}^{\infty}|\hat{f}(\lambda)|^{2}|C(\lambda)|^{-2} d \lambda .
$$

Therefore, the spherical Fourier transform $f \mapsto \hat{f}$ of $C_{c}^{\infty}(G / / K)$ is uniquely extended to an isometry between $L^{2}(G / / K)=L^{2}\left(\mathbf{R}_{+}, \Delta(x) d x\right)$ and $L^{2}\left(\mathbf{R}_{+}\right.$, $|C(\lambda)|^{-2} d \lambda$ ) (cf. [3, Proposition 3], [17, Theorem 9.2.2.13]).

For $f \in C_{c}^{\infty}(G / / K)$ we define the Abel transform $F_{f}^{s}, s \in \mathbf{R}$, of $f$ as

$$
F_{f}^{s}(x)=e^{\rho(1+s) x} \int_{N} f\left(a_{x} n\right) d n
$$

Then the Euclidean Fourier transform $\left(F_{f}^{s}\right)^{\sim}(\lambda)$ is holomorphic on $\mathrm{F}_{c}$ of exponential type, because $F_{f}^{s}(x) \in C_{c}^{\infty}(\mathbf{R})$, and coincides with the spherical Fourier transform:

$$
\hat{f}(\lambda+i s \rho)=\left(F_{f}^{s}\right)^{\sim}(\lambda), \quad \lambda \in \mathrm{F}_{c}
$$

(cf. $[11, \S 3]$ ). Especially, $F_{f}^{0}$ is even on $\mathbf{R}$. The integral over $N$ in (12) can be explicitly rewritten by using a generalized Weyl type fractional integral operator 
$W_{\mu}^{\sigma}:$ For $\sigma>0$ and $\mu \in \mathbf{C}$, we define $W_{\mu}^{\sigma}(f)(y), y>0$, as

$$
W_{\mu}^{\sigma}(f)(y)=\frac{(-1)^{n}}{\Gamma(\mu+n)} \int_{y}^{\infty} \frac{d^{n} f(x)}{d(\operatorname{ch} \sigma x)^{n}}(\operatorname{ch} \sigma x-\operatorname{ch} \sigma y)^{\mu+n-1} d(\operatorname{ch} \sigma x),
$$

where $n=0$ if $\Re \mu>0$ and $-n<\Re \mu \leq-n+1, n=0,1,2, \cdots$, if $\Re \mu \leq 0$ (see [11, (3.11)]). Koornwinder [11, Corollary 3.3] obtains that for $x>0$,

$$
\begin{aligned}
F_{f}^{0}(x) & =W_{\alpha-\beta}^{1} \circ W_{\beta+1 / 2}^{2}(f)(x) \\
& =c \int_{x}^{\infty} f(s) A(x, s) d s
\end{aligned}
$$

where $A(x, s)$ is given by

$$
\begin{aligned}
& (\operatorname{sh} 2 s)(\operatorname{ch} s)^{\beta-1 / 2}(\operatorname{ch} s-\operatorname{ch} x)^{\alpha-1 / 2} F\left(\frac{1}{2}+\beta, \frac{1}{2}-\beta ; \alpha+\frac{1}{2} ; \frac{\operatorname{ch} s-\operatorname{ch} x}{2 \operatorname{ch} s}\right) \\
= & (\operatorname{sh} 2 s) \int_{x}^{s}(\operatorname{ch} 2 s-\operatorname{ch} 2 w)^{\beta-1 / 2}(\operatorname{ch} w-\operatorname{ch} x)^{\alpha-\beta-1}(\operatorname{sh} w) d w
\end{aligned}
$$

(see $[11,(2.18),(2.19),(3.5)])$. We note that for $0 \leq \gamma \leq s_{\alpha}$ and $0<x \leq s$,

$$
|A(x, s)| \leq c e^{(\rho-2) s}(\operatorname{sh} 2 s)(\operatorname{th} s)^{2 \alpha-1} \leq c e^{\rho s}(\operatorname{th} s)^{2 \alpha} .
$$

In the following, for simplicity, we denote $W_{+}(f)(x)=F_{f}^{1}(|x|)$, that is,

$$
W_{+}(f)(x)=e^{\rho x} W_{\alpha-\beta}^{1} \circ W_{\beta+1 / 2}^{2}(f)(|x|), \quad x \in \mathbf{R}
$$

and for a function $F$ on $\mathbf{R}_{+}$,

$$
W_{-}(F)(x)=W_{-(\beta+1 / 2)}^{2} \circ W_{-(\alpha-\beta)}^{1}\left(e^{-\rho x} F\right), \quad x \in \mathbf{R}_{+} .
$$

Then $W_{-} \circ W_{+}(f)=f$ and $W_{+} \circ W_{-}(F)=F$. For $f \in L^{1}(G / / K), W_{+}(f)$ belongs to $L^{1}(\mathbf{R})$, because (12) and the integral formula for the Iwasawa decomposition of $G$ yield that

$$
\left\|W_{+}(f)\right\|_{L^{1}(\mathbf{R})} \leq\|f\|_{1}
$$

(cf. $[11,(3.5),(2.20)])$. Hence $W_{+}(f)^{\sim}(\lambda), \lambda \in \mathrm{F}$, is well-defined and

$$
\hat{f}(\lambda+i \rho)=W_{+}(f)^{\sim}(\lambda), \quad \lambda \in \mathrm{F} .
$$

For $f, g \in L^{1}(G / / K)$, since $f * g \in L^{1}(G / / K)$ and $(f * g)^{\wedge}(\lambda)=\hat{f}(\lambda) \hat{g}(\lambda)$ (cf. $[3$, Theorem 5]), it follows that

$$
W_{+}(f * g)=W_{+}(f) * W_{+}(g)
$$


where the symbol "*" in the left and right hand sides denotes the convolution on $G$ and on $\mathbf{R}$ respectively. We denote them by the same symbol. We say that a function $F$ on $\mathbf{R}$ is $W_{+}$-smooth if $W_{-}(F)$ is well-defined and continuous. Then, for $W_{+}$-smooth functions $F, G$ on $\mathbf{R}$ with compact support such that $e^{-\rho x} F$ and $e^{-\rho x} G$ are even, it follows that

$$
W_{-}(F * G)=W_{-}(F) * W_{-}(G) .
$$

Let $\left(X_{i},\|\cdot\|_{i}\right), i=1,2$, be normed spaces and $T: X_{1} \rightarrow X_{2}$ a sublinear operator. We use the symbol $\left\|x_{1}\right\|_{1} \approx\left\|T\left(x_{1}\right)\right\|_{2}$ if there exist constants $0<c_{1} \leq c_{2}$ such that $c_{1}\left\|x_{1}\right\|_{1} \leq\left\|T\left(x_{1}\right)\right\|_{2} \leq c_{2}\left\|x_{1}\right\|_{1}$ for all $x_{1} \in X_{1}$. We denote by $[\cdot]$ the Gauss symbol and by $f=O(g)$ the Landau symbol. We use letters $c, C, c_{1}, c_{2}, \cdots$ to denote many different constants.

\section{Maximal functions and a reduction formula}

We shall introduce a radial maximal function $M_{\phi} f$ on $G$ and give a reduction formula, which relates $M_{\phi} f$ with a maximal function of $W_{+}(f)$ on $\mathbf{R}$. We suppose that $\phi$ is a positive $C^{\infty}, K$-bi-invariant function on $G$, such that after identifying it with an even function on $\mathbf{R}$, it is supported on $[-1,1]$,

$$
\int_{0}^{\infty} \phi(x) \Delta(x) d x=1, \quad \int_{0}^{1} \phi(x) x \Delta(x) d x \leq \frac{1}{4},
$$

and furthermore, there exists $M \in \mathbf{N}$ such that

$$
\phi(x)=O\left(x^{2 M}\right) .
$$

For $t>0$ we define a dilation $\phi_{t}$ of $\phi$ as

$$
\phi_{t}(x)=\frac{1}{t} \frac{1}{\Delta(x)} \Delta\left(\frac{x}{t}\right) \phi\left(\frac{x}{t}\right) .
$$

As mentioned in $\S 1$ this dilation keeps the $L^{1}$-norm of $\phi$ and gives an approximate identity in $L^{p}(G / / K), 1 \leq p \leq \infty$. We here introduce the radial maximal function $M_{\phi} f$ on $G$ as follows.

Definition 3.1. For $f \in L_{\text {loc }}^{1}(G / / K)$,

$$
\left(M_{\phi} f\right)(g)=\sup _{0<t<\infty}\left|\left(f * \phi_{t}\right)(g)\right|, \quad g \in G .
$$

As shown in [7, Theorem 3.4 and Theorem 3.5], $M_{\phi}$ satisfies the maximal theorem and $\|f\|_{p} \leq\left\|M_{\phi} f\right\|_{p}$, if the both sides exist, for $1 \leq p \leq \infty$. Next, by using $W_{+}\left(\phi_{t}\right)$, we define a maximal function on $\mathbf{R}$ as follows. 
Definition 3.2. For $F \in L_{\text {loc }}^{1}(\mathbf{R})$,

$$
\left(M_{\phi}^{\mathbf{R}} F\right)(x)=\sup _{0<t<\infty}\left|\left(F * W_{+}\left(\phi_{t}\right)\right)(x)\right|, \quad x \in \mathbf{R} .
$$

Since $W_{+}\left(f * \phi_{t}\right)=W_{+}(f) * W_{+}\left(\phi_{t}\right)$ (see $\left.(22)\right)$ and $W_{+}$is an integral operator with a positive kernel (see (15), (16)), it follows that

$$
\begin{aligned}
\sup _{0<t<\infty}\left|W_{+}(f) * W_{+}\left(\phi_{t}\right)(x)\right| & =\sup _{0<t<\infty}\left|W_{+}\left(f * \phi_{t}\right)(x)\right| \\
& \leq W_{+}\left(\sup _{0<t<\infty}\left|f * \phi_{t}\right|\right)(x) .
\end{aligned}
$$

This fact and (20) yield a relation between $M_{\phi}$ and $M_{\phi}^{\mathbf{R}}$ :

Proposition 3.3. For $f \in L_{\mathrm{loc}}^{1}(G / / K)$,

$$
\left(M_{\phi}^{\mathbf{R}} W_{+}(f)\right)(x) \leq W_{+}\left(M_{\phi} f\right)(x), \quad x \in \mathbf{R} .
$$

In particular,

$$
\left\|M_{\phi}^{\mathbf{R}} W_{+}(f)\right\|_{L^{1}(\mathbf{R})} \leq c\left\|M_{\phi} f\right\|_{1}
$$

if the both sides exist.

In what follows we shall prove that the maximal operator $M_{\phi}^{\mathbf{R}}$ characterizes $H^{1}(\mathbf{R})$, that is, $F \in H^{1}(\mathbf{R})$ if and only if $M_{\phi}^{\mathbf{R}}(F) \in L^{1}(\mathbf{R})$ (see Theorem 3.7 below). We first obtain some properties of $W_{+}\left(\phi_{t}\right) \sim(\lambda)=\hat{\phi}_{t}(\lambda+i \rho)$ (see (21)), which guarantee that $W_{+}\left(\phi_{t}\right)$ behaves like a dilation on $\mathbf{R}$. Let $M$ be the same in (24) and $N \in \mathbf{N}$.

LEmma 3.4. There exists a constant $c$ such that for all $t>0, \lambda \in \mathbf{R}, 0 \leq$ $n \leq N$ and $0 \leq k \leq M$,

$$
\left|\left(\frac{d}{d \lambda}\right)^{n} \hat{\phi}_{t}(\lambda+i \rho)\right| \leq c t^{n}(1+t)^{k}(1+|t \lambda|)^{-2 k} .
$$

Proof. For simplicity, we denote $\psi=\Delta \phi$ and $\psi_{[t]}=\phi_{t}$, that is

$$
\psi_{[t]}(x)=\frac{1}{t \Delta(x)} \psi\left(\frac{x}{t}\right)
$$

and moreover, we put for $u, j \in \mathbf{N}$

$$
\psi_{(j)}^{(u)}(x)=\left(\frac{d}{d x}\right)^{u} \psi(x) \cdot x^{j}
$$


We recall that, by identifying a $K$-bi-invariant function $f$ on $G$ with an even function on $\mathbf{R}$, the Laplace-Beltrami operator $\Omega$ acts on $f$ as $\Omega(f)=\Delta^{-1}\left(\Delta f^{\prime}\right)^{\prime}=\mathbf{D} \cdot f^{\prime}+f^{\prime \prime}$, where

$$
\mathbf{D}(x)=\Delta^{\prime}(x) \Delta(x)^{-1}=m_{1}(\operatorname{cth} x)+2 m_{2}(\operatorname{cth} 2 x)
$$

(see (4), (6)). Then it is easy to see that for each $k$,

$$
\left(\Omega^{k} \phi_{t}\right)(x)=\frac{1}{t^{2 k}}\left(\sum_{u=0}^{2 k}\left(\phi_{(u-2 k)}^{(u)}\right)_{[t]}(x) Q_{u}^{k}(x)\right),
$$

where $Q_{u}^{k}(x)$ is a polynomial with degree $\leq k$ of $(d / d x)^{\ell-1} \mathbf{D}(x) \cdot x^{\ell}(1 \leq \ell \leq k)$. We note that

$$
\left|\left(\frac{d}{d x}\right)^{\ell-1} \mathbf{D}(x) \cdot x^{\ell}\right| \leq c \begin{cases}1+|x| & \text { if } \ell=1 \\ 1 & \text { if } \ell>1 .\end{cases}
$$

Since $\phi$ is supported on $[-1,1]$, we may suppose that $|x| \leq t$. Thereby, we can deduce that $\left|Q_{u}^{k}(x)\right| \leq c(1+t)^{k}$. Then the argument used in the proof of $[8$, Lemma 6.5] yields the desired estimate.

LEMmA 3.5. There exists a constant $c$ such that for all $t>1, \lambda \in \mathbf{R}$, and $0 \leq n \leq N$,

$$
\left|\left(\frac{d}{d \lambda}\right)^{n} \hat{\phi}_{t}(\lambda+i \rho)\right| \leq c t^{n}(1+|t \lambda|)^{-(2 M+\alpha+1 / 2)}
$$

Proof. Substituting the Harish-Chandra and Gangolli expansions of $\varphi_{\lambda}$ (see (8) and (10) respectively) with (11), we can expand $\hat{\phi}_{t}(\lambda)$ as

$$
\begin{aligned}
\hat{\phi}_{t}(\lambda) & =\frac{1}{t} \int_{0}^{\infty} \Delta\left(\frac{x}{t}\right) \phi\left(\frac{x}{t}\right) \varphi_{\lambda}(x) d x=\int_{0}^{1} \Delta(x) \phi(x) \varphi_{\lambda}(t x) d x \\
& =\int_{0}^{1} \Delta(x) \phi(x) e^{-\rho t x}\left(\Phi(\lambda, t x) C(\lambda) e^{i \lambda t x}+\Phi(-\lambda, t x) C(-\lambda) e^{-i \lambda t x}\right) d x \\
& =I(\lambda)+I(-\lambda),
\end{aligned}
$$

where

$$
\begin{aligned}
I(\lambda) & =\int_{0}^{1} \Delta(x) \phi(x) e^{-\rho t x} \Phi(\lambda, t x) C(\lambda) e^{i \lambda t x} d x \\
& =\sum_{m=0}^{\infty} \Gamma_{m}(\lambda) C(\lambda) \int_{0}^{1} \Delta(x) \phi(x) e^{-\rho t x} e^{-2 m t x} e^{i \lambda t x} d x \\
& =\sum_{m=0}^{\infty} \Gamma_{m}(\lambda) C(\lambda) I_{m}(\lambda)
\end{aligned}
$$


In order to obtain the desired estimate of $\hat{\phi}_{t}(\lambda+i \rho)$, we first estimate $I_{m}(\xi)$, $\xi= \pm(\lambda+i \rho)$, in (25) and combine it with estimates for $\Gamma_{m}(\xi)$ and $C(\xi)$. Then we sum up these estimates of $\Gamma_{m}(\xi) C(\xi) I_{m}(\xi)$ with respect to $m$.

As for $I_{m}(\lambda+i \rho)$, since $(\phi \Delta)(x)=O\left(x^{2 M+2 \alpha+1}\right)$ (see (4), (24)), integration by parts yields that for $0 \leq l \leq 2 M$,

$$
\begin{aligned}
& (i t \lambda)^{l}\left(\frac{d}{d \lambda}\right)^{n} I_{m}(\lambda+i \rho) \\
= & (i t \lambda)^{l} \int_{0}^{1}(\phi \Delta)(x) e^{-2(m+\rho) t x}(i t x)^{n} e^{i \lambda t x} d x \\
= & (i t)^{n} \int_{0}^{1}(\phi \Delta)(x) x^{n} e^{-2(m+\rho) t x} \cdot\left(\frac{d}{d x}\right)^{l} e^{i \lambda t x} d x \\
= & (i t)^{n}(-1)^{l} \int_{0}^{1}\left(\frac{d}{d x}\right)^{l}\left((\phi \Delta)(x) \cdot x^{n} e^{-2(m+\rho) t x}\right) e^{i \lambda t x} d x
\end{aligned}
$$

and it is dominated by

$$
c t^{n} \sum_{p=0}^{l}((m+\rho) t)^{l-p} \int_{0}^{1}\left|\left(\frac{d}{d x}\right)^{p}\left((\phi \Delta)(x) x^{n}\right)\right| e^{-2(m+\rho) t x} d x .
$$

Let $0<\delta<1$ and set $\beta_{\delta}(p)=(l-p)+2 \alpha+1+\delta(0 \leq p \leq l)$. We take a constant $C$ such that $|x|^{\beta_{\delta}(p)} e^{-x} \leq C$ for all $x \in \mathbf{R}_{+}$and $0 \leq p \leq l$. Then, since $2(m+\rho) \geq 1$ for all $m,(d / d x)^{p}\left((\phi \Delta)(x) x^{n}\right) \cdot e^{-2(m+\rho) t x}$ in $(26)$ is estimated for $0<x<1$ as

$$
\begin{aligned}
& \left|\left(\frac{d}{d x}\right)^{p}\left((\phi \Delta)(x) x^{n}\right)\right| \cdot C|2(m+\rho) t x|^{-\beta_{\delta}(p)} \\
\leq & c C|x|^{2 M+2 \alpha+1+n-p-\beta_{\delta}(p)}((m+\rho) t)^{-\beta_{\delta}(p)} \\
\leq & c C|x|^{-\delta}((m+\rho) t)^{-\beta_{\delta}(p)}
\end{aligned}
$$

Therefore, the integral over $[0,1]$ is finite and $(26)$ is dominated by $t^{n-(2 \alpha+1+\delta)}$ $(m+\rho)^{-(2 \alpha+1+\delta)}$. Hence, we have

$$
\left|\left(\frac{d}{d \lambda}\right)^{n} I_{m}(\lambda+i \rho)\right| \leq c t^{n-(2 \alpha+1+\delta)}(m+\rho)^{-(2 \alpha+1+\delta)}(1+|t \lambda|)^{-2 M} .
$$

As for $I_{m}(-(\lambda+i \rho))$, we can repeat the exactly same process after replacing $e^{-2(m+\rho) t x}$ by $e^{-2 m t x}$. However, when $m=0$, we have no exponential term in (26) and thus, we cannot take $\beta_{\delta}(p)$ for $I_{0}(-(\lambda+i \rho))$ as before. In this case we note that $I_{0}(-(\lambda+i \rho))$ is nothing but the Euclidean Fourier transform $(\phi \Delta)^{\sim}(t \lambda)$ of $(\phi \Delta)(x)$ and thus, it is rapidly decreasing with respect to $|t \lambda|$. Hence, we have for 
arbitrary $n^{\prime} \geq 0$,

$$
\begin{aligned}
& \left|\left(\frac{d}{d \lambda}\right)^{n} I_{m}( \pm(\lambda+i \rho))\right| \\
\leq & c t^{n} \begin{cases}t^{-(2 \alpha+1+\delta)}(m+\rho)^{-(2 \alpha+1+\delta)}(1+|t \lambda|)^{-2 M} & \text { if } m>0 \\
(1+|t \lambda|)^{-n^{\prime}} & \text { if } m=0 .\end{cases}
\end{aligned}
$$

On the other hand, for $\xi= \pm(\lambda+i \rho), \Gamma_{m}(\xi)$ and $C(\xi)$ satisfy the following estimates (see Proposition 8.2 in $\S 8$ and [3, Theorem 2]): For each $n \in \mathbf{N}$

$$
\begin{aligned}
& \left|\left(\frac{d}{d \lambda}\right)^{n} \Gamma_{m}( \pm(\lambda+i \rho))\right| \leq c(1+m)^{2 \alpha+\delta / 2}, \\
& \left|\left(\frac{d}{d \lambda}\right)^{n} C( \pm(\lambda+i \rho))\right| \leq c(1+|\lambda|)^{-(\alpha+1 / 2+n)}
\end{aligned}
$$

Therefore, letting $n^{\prime}=2 M+\alpha+1 / 2$ and substituting (27), (28) and (29) into (25), we see that for $t>1$

$$
\begin{aligned}
\left|\left(\frac{d}{d \lambda}\right)^{n} I( \pm(\lambda+i \rho))\right| & \leq c t^{n}(1+|t \lambda|)^{-(2 M+\alpha+1 / 2)} \sum_{m=0}^{\infty}(1+m)^{-(1+\delta / 2)} \\
& \leq c t^{n}(1+|t \lambda|)^{-(2 M+\alpha+1 / 2)}
\end{aligned}
$$

This completes the proof of the lemma.

Lemma 3.6. Let notations be as above.

(1) $\hat{\phi}_{t}(\lambda+i \rho) \rightarrow 1$ as $|t \lambda| \rightarrow 0$,

(2) $\left|\hat{\phi}_{t}(\lambda+i \rho)\right| \geq 1 / 2$ if $0 \leq|t \lambda| \leq 2$.

Proof. (1) We note that $\hat{\phi}_{t}(\lambda+i \rho)=\int_{0}^{1} \phi(x) \varphi_{\lambda+i \rho}(t x) \Delta(x) d x$ and $\varphi_{i \rho} \equiv 1$. Hence $\hat{\phi}_{t}(i \rho)=1$ by (23) and it follows from [3, Lemma 11, Lemma 14] that

$$
\begin{aligned}
\left|\hat{\phi}_{t}(\lambda+i \rho)-1\right| & =\left|\int_{0}^{1} \phi(x) \Delta(x)\left(\varphi_{\lambda+i \rho}(t x)-\varphi_{i \rho}(t x)\right) d x\right| \\
& \leq|\lambda| \int_{0}^{1} \phi(x) \Delta(x)\left|\left(\frac{d}{d \lambda}\right) \varphi_{\lambda+i \rho}(t x)\right|_{\lambda=\lambda_{0}} \mid d x, \quad 0 \leq \lambda_{0} \leq \lambda \\
& \leq|t \lambda| \int_{0}^{1} \phi(x) x \Delta(x) d x .
\end{aligned}
$$

(2) We note that the last term is dominated by $|t \lambda| / 4$ (see (23)). Therefore, if $0 \leq|t \lambda| \leq 2$, then $\left|\hat{\phi}_{t}(\lambda+i \rho)\right|=\left|\left(\hat{\phi}_{t}(\lambda+i \rho)-1\right)+1\right| \geq-1 / 2+1=1 / 2$.

We here put $\ell(t, \lambda)=\hat{\phi}_{t}(\lambda+i \rho)=W_{+}\left(\phi_{t}\right) \sim(\lambda)$ and refer to $\S 8$. B. Then Lemmas $3.4,3.5$, and 3.6 imply that $\ell(t, \lambda)$ belongs to the class $A_{N, 2 M}$ for all 
$N \in \mathbf{N}$ (see Definition 8.4) and, furthermore, Lemma 3.6 (2) implies that $\ell(t, \lambda)$ satisfies the assumption (90) in Theorem 8.6. Since $M_{\ell}=M_{\phi}^{\mathbf{R}}$ (see Definitions 3.2 and 8.5 ), Theorem 8.6 yields the following.

TheOREM 3.7. Let $\phi$ be as above and suppose that $M \geq 2$. Then $F \in H^{1}(\mathbf{R})$ if and only if $M_{\phi}^{\mathbf{R}} F \in L^{1}(\mathbf{R})$ :

$$
\|F\|_{H^{1}(\mathbf{R})} \approx\left\|M_{\phi}^{\mathbf{R}} F\right\|_{L^{1}(\mathbf{R})} .
$$

\section{Real Hardy spaces on $G$}

Let $\phi$ be the same as in the previous section (see (23), (24)) and $M_{\phi}, M_{\phi}^{\mathbf{R}}$ the corresponding radial maximal operators on $G$ and $\mathbf{R}$ respectively (see Definitions 3.1 and 3.2). In this section we shall define two real Hardy spaces $H_{\phi}^{1}(G / / K)$ and $W_{-}\left(H^{1}(\mathbf{R})\right)$ on $G$ and give a relation between them. Especially, we shall give a simple proof of Theorem A based on fractional calculus.

We introduce the real Hardy space $H_{\phi}^{1}(G / / K)$ on $G$ as follows.

Definition 4.1. We define

$$
H_{\phi}^{1}(G / / K)=\left\{f \in L_{\mathrm{loc}}^{1}(G / / K) ; M_{\phi} f \in L^{1}(G / / K)\right\}
$$

and $\|f\|_{H_{\phi}^{1}(G)}=\left\|M_{\phi} f\right\|_{1}$.

Since $\|f\|_{1} \leq\left\|M_{\phi} f\right\|_{1}$, it follows that

$$
H_{\phi}^{1}(G / / K) \subset L^{1}(G / / K)
$$

Let $\mathrm{M}_{s}, s \geq 0$, denote the Euclidean Fourier multiplier corresponding to $(\lambda+i \rho)^{s}$ :

$$
\mathrm{M}_{s}(F)^{\sim}(\lambda)=(\lambda+i \rho)^{s} F^{\sim}(\lambda) .
$$

We introduce a pull-back of $H^{1}(\mathbf{R})$ to $G$ via $W_{+}$(see (18)) and $\mathrm{M}_{s}$ :

Definition 4.2. For $s \geq 0$, we define

$$
W_{-}\left(\mathrm{M}_{-s}\left(H^{1}(\mathbf{R})\right)\right)=\left\{f \in L_{\mathrm{loc}}^{1}(G / / K) ; \mathrm{M}_{s} \circ W_{+}(f) \in H^{1}(\mathbf{R})\right\}
$$

and give the norm by $\left\|\mathrm{M}_{s} \circ W_{+}(f)\right\|_{H^{1}(\mathbf{R})}$. We denote $W_{-}\left(\mathrm{M}_{0}\left(H^{1}(\mathbf{R})\right)\right)$ by $W_{-}\left(H^{1}(\mathbf{R})\right)$.

An easy consequence of Proposition 3.3 and Theorem 3.7 is the following.

Corollary 4.3. Let $M \geq 2$. There exists a positive constant $c$ such that $\left\|W_{+}(f)\right\|_{H^{1}(\mathbf{R})} \leq c\|f\|_{H_{\phi}^{1}(G)}$ for all $f \in H_{\phi}^{1}(G / / K)$ and thus,

$$
H_{\phi}^{1}(G / / K) \subset W_{-}\left(H^{1}(\mathbf{R})\right) .
$$


We shall conversely control the $H_{\phi}^{1}$-norm of $f$ by using $W_{+}(f)$. Before starting the argument we shall obtain some basic properties of the Euclidean fractional integral operators $W_{\mu}^{\mathbf{R}}$ and $\tilde{W}_{\mu}^{\mathbf{R}}$ on $\mathbf{R}_{+}$, which correspond to the case of $\alpha=\beta=$ $-1 / 2$ and $\sigma=1$ in (14) and (92) respectively: For $\mu \in \mathbf{C}$ and $y>0$,

$$
W_{\mu}^{\mathbf{R}}(f)(y)=\frac{(-1)^{n}}{\Gamma(\mu+n)} \int_{y}^{\infty} \frac{d^{n} f(x)}{d x^{n}}(x-y)^{\mu+n-1} d x
$$

and

$$
\tilde{W}_{\mu}^{\mathbf{R}}(f)(y)=\frac{1}{\Gamma(\mu+n)} \frac{d^{n}}{d y^{n}} \int_{0}^{y} f(x)(x-y)^{\mu+n-1} d x,
$$

where $n=0$ if $\Re \mu>0$ and $-n<\Re \mu \leq-n+1, n=0,1,2, \cdots$, if $\Re \mu \leq 0$. Let $0 \leq \mu<1$ and let $f$ be an integrable function on $\mathbf{R}_{+}$. Then, by changing the order of integration and by using integration by parts, we see that $W_{\mu}^{\mathbf{R}} \circ W_{-\mu}^{\mathbf{R}}(f)=f$ if $f^{\prime}(x) x^{-\mu}$ is integrable, and $W_{-\mu}^{\mathbf{R}} \circ W_{\mu}^{\mathbf{R}}(f)=f$ if $f(x) x^{\mu-1}$ is integrable. Moreover, if $f, g$ are integrable functions on $\mathbf{R}_{+}$such that $f(x) x^{\mu-1}, g^{\prime}$ are integrable and $g(0)=\lim _{x \rightarrow \infty} g^{\prime}(x)=0$, then

$$
\langle f, g\rangle_{L^{1}\left(\mathbf{R}_{+}\right)}=\left\langle W_{\mu}^{\mathbf{R}}(f), \tilde{W}_{-\mu}^{\mathbf{R}}(g)\right\rangle .
$$

Lemma 4.4. Let $f, f^{\prime}$ be integrable on $x \geq 0$ and $0 \leq \mu<1$.

(1) For $\gamma \leq 0$,

$$
\int_{x}^{\infty} f^{\prime}(s) s^{\gamma}(s-x)^{-\mu} d s=c W_{-\mu}^{\mathbf{R}}(f)(x) x^{\gamma}+\int_{x}^{\infty} W_{-\mu}^{\mathbf{R}}(f)(s) B_{\gamma}(x, s) d s,
$$

where $B_{\gamma}(x, s)$ is smooth on $0<x \leq s$ and there exists a constant $c>0$ such that

$$
\left|B_{\gamma}(x, s)\right| \leq c x^{\gamma-1} \quad \text { for all } \quad 0<x \leq s
$$

(2) For $\gamma>0$,

$$
\int_{x}^{\infty} f^{\prime}(s) e^{-\gamma s}(s-x)^{-\mu} d s=c e^{-\gamma x}\left(W_{-\mu}^{\mathbf{R}}(f)(x)+\int_{x}^{\infty} f(s) C(x, s) d s\right),
$$

where $C(x, s)$ is smooth on $0<x \leq s$ and there exists a constant $c>0$ such that

$$
\int_{0}^{s}|C(x, s)| d x \leq c \text { for all } s>0 .
$$

(3) Let $g$ be a smooth function.

$$
W_{-\mu}^{\mathbf{R}}(f \cdot g)(x)=c W_{-\mu}^{\mathbf{R}}(f)(x) \cdot g(x)+\int_{x}^{\infty} W_{-\mu}^{\mathbf{R}}(f)(s) D_{g}(x, s) d s,
$$


where $D_{g}(x, s)$ is smooth on $0<x \leq s$ and there exists a constant $c>0$ such that

$$
\left|D_{g}(x, s)\right| \leq c \sup _{x \leq t \leq s}\left|g^{\prime}(t)\right| \text { for all } 0<x \leq s .
$$

Especially, if $g$ is supported on $[0,1]$ or $g$ is constant on $[1, \infty)$, then $D_{g}(x, s)=0$ for $1 \leq x \leq s$.

(4) For $\gamma>0$,

$$
W_{-\mu}^{\mathbf{R}}\left(e^{-\gamma x} f\right)(x)=c e^{-\gamma x}\left(W_{-\mu}^{\mathbf{R}}(f)(x)+\int_{x}^{\infty} f(s) C(x, s) d s\right),
$$

where $C(x, s)$ is smooth on $0<x \leq s$ and satisfies (34).

Proof. (1): We note that

$$
\begin{aligned}
& \int_{x}^{\infty} f^{\prime}(s) s^{\gamma}(s-x)^{-\mu} d s \\
= & \int_{x}^{\infty}\left(f^{\prime}(s) x^{\gamma}+f^{\prime}(s)\left(s^{\gamma}-x^{\gamma}\right)\right)(s-x)^{-\mu} d s \\
= & c W_{-\mu}^{\mathbf{R}}(f)(x) x^{\gamma}-\int_{x}^{\infty} f(s)\left(\left(s^{\gamma}-x^{\gamma}\right)(s-x)^{-\mu}\right)^{\prime} d s \\
= & c W_{-\mu}^{\mathbf{R}}(f)(x) x^{\gamma}+\int_{x}^{\infty} W_{-\mu}^{\mathbf{R}}(f)(s) B_{\gamma}(x, s) d s,
\end{aligned}
$$

where

$$
\begin{aligned}
B_{\gamma}(x, s) & =-\tilde{W}_{\mu}^{\mathbf{R}}\left(\left(\left(s^{\gamma}-x^{\gamma}\right)(s-x)^{-\mu}\right)^{\prime} \chi_{[x, \infty)}\right)(s) \\
& =-\int_{x}^{s}\left(\gamma t^{\gamma-1}(t-x)^{-\mu}-\mu\left(t^{\gamma}-x^{\gamma}\right)(t-x)^{-\mu-1}\right)(s-t)^{\mu-1} d t
\end{aligned}
$$

Since $\gamma-1<0$ and $\left(t^{\gamma}-x^{\gamma}\right) /(t-x) \leq \gamma x^{\gamma-1}$ for $0<x<t$, it follows that

$$
\left|B_{\gamma}(x, s)\right| \leq c x^{\gamma-1} \int_{x}^{s}(t-x)^{-\mu}(s-t)^{\mu-1} d t \sim x^{\gamma-1}
$$

(2): We note that

$$
\begin{aligned}
& \int_{x}^{\infty} f^{\prime}(s) e^{-\gamma s}(s-x)^{-\mu} d s \\
= & \int_{x}^{\infty} f^{\prime}(s) e^{-\gamma x}\left(1+\left(e^{-\gamma(s-x)}-1\right)\right)(s-x)^{-\mu} d s \\
= & c e^{-\gamma x}\left(W_{-\mu}^{\mathbf{R}}(f)(x)+\int_{x}^{\infty} f(s) C(x, s) d s\right),
\end{aligned}
$$


where

$$
C(x, s)=\left(\left(e^{-\gamma(s-x)}-1\right)(s-x)^{-\mu}\right)^{\prime} \chi_{[x, \infty)}(s)
$$

Therefore,

$$
\begin{aligned}
\int_{0}^{s}|C(x, s)| d x & =c \int_{x}^{s}\left|\left(\left(e^{-\gamma(t-x)}-1\right)(t-x)^{-\mu}\right)^{\prime}\right| d t \\
& \leq c \sup _{x \leq t \leq s}\left|\left(e^{-\gamma(t-x)}-1\right)(t-x)^{-\mu}\right| \leq c
\end{aligned}
$$

(3): Since

$$
\begin{aligned}
(f(s) g(s))^{\prime} & =f^{\prime}(s) g(x)+f^{\prime}(s)(g(s)-g(x))+f(s) g^{\prime}(s) \\
& =f^{\prime}(s) g(x)+(f(s)(g(s)-g(x)))^{\prime}
\end{aligned}
$$

it follows that

$$
\begin{aligned}
& W_{-\mu}^{\mathbf{R}}(f \cdot g)(x) \\
= & \int_{x}^{\infty}(f(s) g(s))^{\prime}(s-x)^{-\mu} d s \\
= & g(x) W_{-\mu}^{\mathbf{R}}(f)+\mu \int_{x}^{\infty} f(s)(g(s)-g(x))(s-x)^{-\mu-1} d s \\
= & g(x) W_{-\mu}^{\mathbf{R}}(f)+\int_{x}^{\infty} W_{-\mu}^{\mathbf{R}}(f)(s) D_{g}(x, s) d s,
\end{aligned}
$$

where

$$
\begin{aligned}
D_{g}(x, s) & =c \tilde{W}_{\mu}^{\mathbf{R}}\left((g(t)-g(x))(t-x)^{-\mu-1} \chi_{[x, \infty)}(t)\right)(s) \\
& =c \int_{x}^{s}\left(\frac{g(t)-g(x)}{t-x}\right)(t-x)^{-\mu}(s-t)^{\mu-1} d t \\
& \leq c \sup _{x \leq t \leq s}\left|g^{\prime}(t)\right| \int_{x}^{s}(t-x)^{-\mu}(s-t)^{\mu-1} d t .
\end{aligned}
$$

Then the desired result follows.

(4): We put $C(x, s)=e^{\gamma x}\left(e^{-\gamma s}-e^{-\gamma x}\right)(s-x)^{-\delta-1}$ in (35). Since

$$
\int_{0}^{s}|C(x, s)| d x=\int_{0}^{s}\left|e^{-\gamma x}-1\right| x^{-\mu-1} d s \leq \int_{0}^{\infty}\left(1-e^{-\gamma x}\right) x^{-\mu-1} d x
$$

$C(x, s)$ satisfies $(34)$. 
We shall deduce the local and global forms of the Weyl type fractional operator

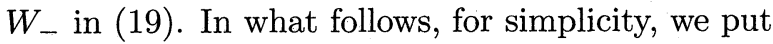

$$
\begin{array}{ll}
\alpha-\beta=n+\delta, & n=[\alpha-\beta], \\
\beta+\frac{1}{2}=n^{\prime}+\delta^{\prime}, & n^{\prime}=\left[\beta-\frac{1}{2}\right] .
\end{array}
$$

Furthermore, we denote

$$
\underline{n}=n+n^{\prime}, \quad \underline{\delta}=\delta+\delta^{\prime}, \quad s_{\alpha}=\alpha+\frac{1}{2}=\underline{n}+\underline{\delta}
$$

and

$$
\underline{D}=\left\{0, \delta, \delta^{\prime}, \delta+\delta^{\prime}\right\} .
$$

Clearly, from the explicit values of $\alpha, \beta$ (cf. [5, Table 1] in p. 265), we may suppose that $\delta=0$. However, for the sake of the Fourier-Jacobi analysis (cf. [4], [11]) we dare not take $\delta=0$.

Proposition 4.5. (1) If $F$ is $W_{+}$-smooth and supported on $0<x \leq 1$, then

$$
\begin{aligned}
& \left|W_{-}(F)(x)\right| \\
\leq & c \sum_{m, \xi}\left(x^{-2 s_{\alpha}+m+\xi} W_{-(m+\xi)}^{\mathbf{R}}(F)(x)+\int_{x}^{\infty}\left|W_{-(m+\xi)}^{\mathbf{R}}(F)(s)\right| A_{m, \xi}^{1}(x, s) d s\right),
\end{aligned}
$$

where the sum is taken over $0 \leq m \leq \underline{n}$ and $\xi \in \underline{D}$, and $A_{m, 0}^{1}(x, s) \equiv 0$ and $A_{m, \xi}^{1}(x, s)$ satisfies

$$
0 \leq A_{m, \xi}^{1}(x, s) \leq x^{-2 s_{\alpha}+m+\xi-1} \quad \text { for all } \quad 0<x \leq s .
$$

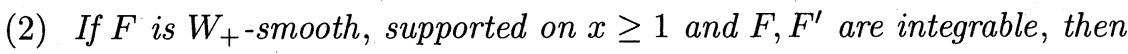

$$
\begin{aligned}
& \left|W_{-}(F)(x)\right| \\
& \leq c \sum_{m, \xi}\left(\left(x^{-2 s_{\alpha}+m+\xi} W_{-(m+\xi)}^{\mathbf{R}}(F)(x)+\int_{x}^{\infty}\left|W_{-(m+\xi)}^{\mathbf{R}}(F)(s)\right| A_{m, \xi}^{2}(x, s) d s\right.\right. \\
& \left.\quad+x^{-2 s_{\alpha}+m+\xi} \int_{x}^{\infty}\left|W_{-(m+\xi)}^{\mathbf{R}}(F)(s)\right| A_{m, \xi}^{3}(x, s) d s\right) \chi_{[0,1]}(x) \\
& \left.\quad+e^{-2 \rho x}\left(W_{-(m+\xi)}^{\mathbf{R}}(F)(x)+\int_{x}^{\infty}\left|W_{-(m+\xi)}^{\mathbf{R}}(F)(s)\right| A_{m, \xi}^{4}(x, s) d s\right) \chi_{[1, \infty)}(x)\right),
\end{aligned}
$$

where $A_{m, 0}^{j}(x, s) \equiv 0$ and $A_{m, \xi}^{j}(x, s) \geq 0$ for $j=2,3,4$, and $A_{m, \xi}^{2}(x, s)$ satisfies 
(39) and there exists a positive constant $c$ such that for $j=3,4$,

$$
\int_{0}^{s} A_{m, \xi}^{j}(x, s) d x \leq c \text { for all } s>0 .
$$

Proof. We shall consider the case of $0<\delta, \delta^{\prime}<1$. Other cases easily follow from the same argument. In the following we use the same letters $B_{\gamma}(x, s)$ and $C(x, s)$ to denote different functions satisfying (33) and (34) respectively.

(1): Let $F$ be differentiable and supported on $[0,1]$. For $\sigma \geq 0$, Lemma 4.4 (1) yields

$$
\begin{aligned}
W_{-\delta}^{\sigma}(F)(x) & =c \int_{x}^{\infty} \frac{d F}{d \operatorname{ch} \sigma x}(s)(\operatorname{ch} \sigma s-\operatorname{ch} \sigma x)^{-\delta} \operatorname{sh} \sigma s d s \\
& \sim \int_{x}^{\infty} F^{\prime}(s)(s-x)^{-\delta}(s+x)^{-\delta} d s \\
& =c x^{-\delta} W_{-\delta}^{\mathbf{R}}(F)(x)+\int_{x}^{\infty} W_{-\delta}^{\mathbf{R}}(F)(s) B_{-\delta}(x, s) d s
\end{aligned}
$$

and for $p \in \mathbf{N}$, Lemma 4.4 (3) gives

$$
W_{-\delta}^{\mathbf{R}}\left(x^{-p} F\right)(x)=c x^{-p} W_{-\delta}^{\mathbf{R}}(F)(x)+\int_{x}^{\infty} W_{-\delta}^{\mathbf{R}}(F)(s) D_{x^{-p}}(x, s) d s .
$$

Therefore, combining these equations, we have

$$
\begin{aligned}
W_{-\delta}^{\sigma}\left(x^{-p} F\right)(x) \sim x^{-(p+\delta)} & W_{-\delta}^{\mathbf{R}}(F)(x)+x^{-\delta} \int_{x}^{\infty} W_{-\delta}^{\mathbf{R}}(F)(s) D_{x^{-p}}(x, s) d s \\
& +\int_{x}^{\infty} s^{-p} W_{-\delta}^{\mathbf{R}}(F)(s) B_{-\delta}(x, s) d s \\
& +\int_{x}^{\infty}\left(\int_{s}^{\infty} W_{-\delta}^{\mathbf{R}}(F)(t) D_{x^{-p}}(s, t) d t\right) B_{-\delta}(x, s) d s
\end{aligned}
$$

Since $\left|D_{x^{-p}}(x, s)\right| \leq c x^{-p-1},\left|B_{-\delta}(x, s)\right| \leq c x^{-\delta-1}$ and

$$
\left|\int_{x}^{t} D_{x^{-p}}(s, t) B_{-\delta}(x, s) d s\right| \leq c x^{-\delta-1} \int_{x}^{t} s^{-p-1} d s \sim x^{-(p+\delta)-1}
$$

for $0<x \leq s$, it follows that

$$
W_{-\delta}^{\sigma}\left(x^{-p} F\right)(x) \sim x^{-(p+\delta)} W_{-\delta}^{\mathbf{R}}(F)(x)+\int_{x}^{\infty} W_{-\delta}^{\mathbf{R}}(F)(s) B_{-(p+\delta)}(x, s) d s .
$$

We here apply $W_{-\delta^{\prime}}^{\sigma^{\prime}}\left(\sigma^{\prime} \geq 0\right)$ to (41). Repeating the above argument, we see that

$$
W_{-\delta^{\prime}}^{\sigma^{\prime}}\left(x^{-(p+\delta)} W_{-\delta}^{\mathbf{R}} F\right)(x)
$$




$$
=c x^{-\left(p+\delta+\delta^{\prime}\right)} W_{-\left(\delta+\delta^{\prime}\right)}^{\mathbf{R}}(F)(x)+\int_{x}^{\infty} W_{-\left(\delta+\delta^{\prime}\right)}^{\mathbf{R}}(F)(s) B_{x^{-\left(p+\delta+\delta^{\prime}\right)}}(x, s) d s
$$

and

$$
\begin{aligned}
& W_{-\delta^{\prime}}^{\sigma^{\prime}}\left(\int_{x}^{\infty} W_{-\delta}^{\mathbf{R}}(F)(s) B_{-(p+\delta)}(x, s) d s\right) \\
= & \int_{x}^{\infty} W_{-\delta}^{\mathbf{R}}(F)(s)\left(W_{-\delta^{\prime}}^{\sigma^{\prime}} B_{-(p+\delta)}\right)(x, s) d s \\
= & \int_{x}^{\infty} W_{-\left(\delta+\delta^{\prime}\right)}^{\mathbf{R}}(F)(s) \tilde{W}_{\delta^{\prime}}^{\mathbf{R}}\left(W_{-\delta^{\prime}}^{\sigma^{\prime}} B_{-(p+\delta)}\right)(x, s) d s .
\end{aligned}
$$

Since

$$
\left|\tilde{W}_{\delta^{\prime}}^{\mathbf{R}}\left(W_{-\delta^{\prime}}^{\sigma^{\prime}} B_{-(p+\delta)}\right)(x, s)\right| \leq c x^{-(p+\delta)-1-2 \delta^{\prime}+\delta^{\prime}}=x^{-\left(p+\delta+\delta^{\prime}\right)-1}
$$

for $0<x \leq s$ (see [10, Lemma 3.1, Lemma 3.3]), it follows that

$$
\begin{aligned}
W_{-\delta^{\prime}}^{\sigma^{\prime}} \circ W_{-\delta}^{\sigma}\left(x^{-p} F\right)(x) \sim & x^{-\left(p+\delta+\delta^{\prime}\right)} W_{-\left(\delta+\delta^{\prime}\right)}^{\mathbf{R}}(F)(x) \\
& +\int_{x}^{\infty} W_{-\left(\delta+\delta^{\prime}\right)}^{\mathbf{R}}(F)(s) B_{-\left(p+\delta+\delta^{\prime}\right)}(x, s) d s .
\end{aligned}
$$

Now let $\gamma=-(\alpha-\beta)=-(n+\delta)$ and $\gamma^{\prime}=-(\beta+1 / 2)=-\left(n^{\prime}+\delta^{\prime}\right)$ (see (36)). Since $W_{-1}^{\sigma}(F)(x)=d F / d(\operatorname{ch} \sigma x) \sim F^{\prime}(x) / x$, it follows that $W_{-n}^{\sigma}(F)(x) \sim$ $\sum_{m=0}^{n} c_{m} x^{-(2 n-m)} F^{(m)}(x)$ and thus

$$
\begin{aligned}
& W_{-n}^{\sigma}\left(e^{-\rho x} F\right)(x) \sim \sum_{m=0}^{n} \sum_{\ell=0}^{m} c_{m \ell} x^{-(2 n-m)} F^{(\ell)}(x), \\
& W_{-n}^{\sigma}\left(x^{-p} F\right)(x) \sim \sum_{m=0}^{n} \sum_{\ell=0}^{m} c_{m \ell} x^{-(2 n-\ell+p)} F^{(\ell)}(x) .
\end{aligned}
$$

Here we note that

$$
W_{\gamma^{\prime}}^{\sigma^{\prime}} \circ W_{\gamma}^{\sigma}=W_{-n^{\prime}}^{\sigma^{\prime}} \circ\left(W_{-\delta^{\prime}}^{\sigma^{\prime}} \circ W_{-\delta}^{\sigma}\right) \circ W_{-n}^{\sigma} .
$$

Then, combining (43), (42) and (44) in this order, we finally obtain that

$$
\begin{aligned}
& W_{\gamma^{\prime}}^{\sigma^{\prime}} \circ W_{\gamma}^{\sigma}\left(e^{-\rho x} F\right)(x) \\
& \sim \sum_{m=0}^{n} \sum_{m^{\prime}=0}^{n^{\prime}} \sum_{\ell=0}^{m} \sum_{\ell^{\prime}=0}^{m^{\prime}}\left(x^{-\left(2 n+2 n^{\prime}-m-\ell^{\prime}+\underline{\delta}\right)} W_{-\left(\ell+\ell^{\prime}+\underline{\delta}\right)}^{\mathbf{R}}(F)(x)\right. \\
& \left.\quad+x^{-\left(2 n^{\prime}-m^{\prime}\right)} \int_{x}^{\infty} W_{-(\ell+\underline{\delta})}^{\mathbf{R}}(F)(s) B_{-\left(2 n-m-m^{\prime}+\underline{\delta}\right)}(x, s) d s\right)
\end{aligned}
$$




$$
\begin{aligned}
\sim \sum_{m=0}^{n} \sum_{m^{\prime}=0}^{n^{\prime}} \sum_{\ell=0}^{m} \sum_{\ell^{\prime}=0}^{m^{\prime}}( & x^{-2 s_{\alpha}+\underline{\delta}+m+\ell^{\prime}} W_{-\left(\ell+\ell^{\prime}+\underline{\delta}\right)}^{\mathbf{R}}(F)(x) \\
& \left.\quad+\int_{x}^{\infty} W_{-(\ell+\underline{\delta})}^{\mathbf{R}}(F)(s) B_{-2 s_{\alpha}+\underline{\delta}+m}(x, s) d s\right) .
\end{aligned}
$$

Since $0 \leq x \leq 1$, we can replace $x^{m}$ and $B_{-2 s_{\alpha}+\underline{\delta}+m}$ by $x^{\ell}$ and $B_{-2 s_{\alpha}+\underline{\delta}+\ell}$ respectively, the desired result follows.

(2): Let $F$ be differentiable and supported on $[1, \infty)$. We keep the notations in (1). It follows from Lemma 4.4 (2) that

$$
\begin{aligned}
W_{-\delta}^{\sigma}(F)(x) & =c \int_{x}^{\infty} \frac{d F}{d \operatorname{ch} \sigma x}(s)(\operatorname{ch} \sigma s-\operatorname{ch} \sigma x)^{-\delta} \operatorname{sh} \sigma s d s \\
& \sim \int_{x}^{\infty} F^{\prime}(x) e^{-\delta \sigma s}\left(1+(s-x)^{-\delta}\right) d s \\
& =c e^{-\delta \sigma x}\left(W_{-\delta}^{\mathbf{R}}(F)(s)+\int_{x}^{\infty} F(s) C(x, s) d s+F(x)\right) .
\end{aligned}
$$

Then, by substituting $F$ with $e^{-\xi x} F$, Lemma 4.4 (4) yields that for $\xi>0$,

$$
\begin{aligned}
& W_{-\delta}^{\sigma}\left(e^{-\xi x} F\right)(x) \\
= & c e^{-(\xi+\delta \sigma) x}\left(W_{-\delta}^{\mathbf{R}}(F)(x)+\int_{x}^{\infty} F(s) C(x, s) d s\right. \\
& \left.\quad+e^{\xi x} \int_{x}^{\infty} F(s) e^{-\xi s} C(x, s) d s+F(x)\right) \\
= & c e^{-(\xi+\delta \sigma) x}\left(W_{-\delta}^{\mathbf{R}}(F)(x)+\int_{x}^{\infty} F(s) C(x, s) d s+F(x)\right) .
\end{aligned}
$$

Let $\Phi(x)$ be an even $C^{\infty}$ function on $\mathbf{R}$ such that $0 \leq \Phi(x) \leq 1, \Phi(x) \equiv 1$ if $|x| \leq 1 / 2$, and $\Phi(x) \equiv 0$ if $|x| \geq 1$. We put

$$
W_{-\delta}^{\sigma}\left(e^{-\xi x} F\right)=W_{-\delta}^{\sigma}\left(e^{-\xi x} F\right)(1-\Phi)+W_{-\delta}^{\sigma}\left(e^{-\xi x} F\right) \Phi .
$$

We apply $W_{-\delta^{\prime}}^{\sigma^{\prime}}$ to each term in (48). We first substitute (47) into (48) and, to each resultant term we apply Lemma $4.4(3)$ and (47) with $\sigma, \delta, \xi$ replaced by $\sigma^{\prime}, \delta^{\prime}, \xi+\delta \sigma$ :

$$
\begin{aligned}
& W_{-\delta^{\prime}}^{\sigma^{\prime}}\left(e^{-(\xi+\delta \sigma) x} W_{-\delta}^{\mathbf{R}}(F)(1-\Phi)\right) \\
& \sim e^{-\left(\xi+\delta \sigma+\delta^{\prime} \sigma^{\prime}\right) x}\left(W_{-\left(\delta+\delta^{\prime}\right)}^{\mathbf{R}}(F)(x)\right. \\
&\left.\quad+\int_{x}^{\infty} W_{-\delta}^{\mathbf{R}}(F)(s) C(x, s) d s+W_{-\delta}^{\mathbf{R}}(F)(x)\right)(1-\Phi)+K_{1}(x)
\end{aligned}
$$




$$
\begin{aligned}
\sim e^{-\left(\xi+\delta \sigma+\delta^{\prime} \sigma^{\prime}\right) x}( & W_{-\left(\delta+\delta^{\prime}\right)}^{\mathbf{R}}(F)(x) \\
& \left.+\int_{x}^{\infty}\left(W_{-\delta}^{\mathbf{R}}(F)(s)+W_{-\underline{\delta}}^{\mathbf{R}}(F)(s)\right) C(x, s) d s+W_{-\delta}^{\mathbf{R}}(F)(x)\right),
\end{aligned}
$$

where, if we denote the first term in the right hand side of $(49)$ by $I_{1}(x)(1-\Phi)$, then $K_{1}(x)$ is given by $K_{1}(x)=\int_{x}^{s} I_{1}(s) D_{1-\Phi}(x, s) d s$. Here, to deduce the last line, we used Lemma $4.4(3)$ and the fact that $\int_{x}^{t} C(s, t) D_{1-\Phi}(x, s) d s$ satisfies (34). Similarly, we have

$$
\begin{aligned}
& W_{-\delta}^{\sigma^{\prime}}\left(e^{-(\xi+\delta \sigma) x} \int_{x}^{\infty} F(s) C(x, s) d s \cdot(1-\Phi)\right) \\
\sim & e^{-\left(\xi+\delta \sigma+\delta^{\prime} \sigma^{\prime}\right) x}\left(\int_{x}^{\infty} F(s)\left(W_{-\delta^{\prime}}^{\mathbf{R}} C\right)(x, s) d s+\int_{x}^{\infty}\left(\int_{s}^{\infty} F(t) C(s, t) d t\right) C(x, s) d s\right. \\
& \left.\quad+\int_{x}^{\infty} F(s) C(x, s) d s\right)(1-\Phi)+K_{2}(x) \\
\sim & e^{-\left(\xi+\delta \sigma+\delta^{\prime} \sigma^{\prime}\right) x}\left(\int_{x}^{\infty} W_{-\delta^{\prime}}^{\mathbf{R}}(F)(s) C(x, s) d s+\int_{x}^{\infty} F(s) C(x, s) d s\right)
\end{aligned}
$$

where, if we denote the first term in the right hand side of $(50)$ by $I_{2}(x)(1-\Phi)$, then $K_{2}(x)$ is given by $K_{2}(x)=\int_{x}^{s} I_{2}(s) D_{1-\Phi}(x, s) d s$. In this case, to deduce the last line, we used Lemma $4.4(3),(32)$ and the facts that $\tilde{W}_{\delta^{\prime}}^{\mathbf{R}} \circ W_{-\delta^{\prime}}^{\mathbf{R}}(C) \sim C$ (see [10, Lemma 3.1]) and $\int_{x}^{t} C(s, t) C(x, s) d s$ satisfies (34). Therefore, we see that

$$
\begin{aligned}
W_{-\delta^{\prime}}^{\sigma^{\prime}}\left(W_{-\delta}^{\sigma}\left(e^{-\xi x} F\right)(1-\Phi)\right) & \\
\sim e^{-\left(\xi+\delta \sigma+\delta^{\prime} \sigma^{\prime}\right) x} & \left(W_{-\left(\delta+\delta^{\prime}\right)}^{\mathbf{R}}(F)(x)+W_{-\delta}^{\mathbf{R}}(F)(x)+W_{-\delta^{\prime}}^{\mathbf{R}}(F)(x)\right. \\
& \left.+\int_{x}^{\infty}\left(W_{-\delta}^{\mathbf{R}}(F)(s)+W_{-\delta^{\prime}}^{\mathbf{R}}(F)(s)+W_{-\underline{\delta}}^{\mathbf{R}}(F)(s)+F(s)\right) C(x, s) d s\right) .
\end{aligned}
$$

As for $W_{-\delta^{\prime}}^{\sigma^{\prime}}\left(W_{-\delta}^{\sigma}\left(e^{-\xi x} F\right) \Phi\right)$, we use $(47)$ and note that $W_{-\delta}^{\sigma}\left(e^{-\xi x} F\right) \Phi$ is supported on $[0,1]$. Hence, (46) with $\gamma, \rho$ replaced by $0, \xi+\delta \sigma$ respectively yields that

$$
\begin{aligned}
& W_{-\delta^{\prime}}^{\sigma^{\prime}}\left(W_{-\delta}^{\sigma}\left(e^{-\xi x} F\right) \Phi\right) \\
= & W_{-\delta^{\prime}}^{\sigma^{\prime}}\left(e^{-(\xi+\delta \sigma) x}\left(W_{-\delta}^{\mathbf{R}}(F)(x)+\int_{x}^{\infty} F(s) C(x, s) d s+F(x)\right) \Phi\right) \\
\sim & \left(x^{-\delta^{\prime}} W_{-\underline{\delta}}^{\mathbf{R}}(F)(x)+\int_{x}^{\infty} W_{-\underline{\delta}}^{\mathbf{R}}(F)(s) B_{-\delta^{\prime}}(x, s) d s\right. \\
& \quad+x^{-\delta^{\prime}} \int_{x}^{\infty} F(s)\left(W_{-\delta^{\prime}}^{\mathbf{R}} C\right)(x, s) d s
\end{aligned}
$$




$$
\begin{aligned}
+ & \int_{x}^{\infty}\left(\int_{s}^{\infty} F(t)\left(W_{-\delta^{\prime}}^{\mathbf{R}} C\right)(s, t) d t\right) B_{-\delta^{\prime}}(x, s) d s \\
+ & \left.x^{-\delta^{\prime}} W_{-\delta^{\prime}}^{\mathbf{R}}(F)(x)+\int_{x}^{\infty} W_{-\delta^{\prime}}^{\mathbf{R}}(F)(s) B_{-\delta^{\prime}}(x, s) d s\right) \Phi \\
& +K_{3}(x) \\
\sim x^{-\delta^{\prime}} & \left(W_{-\delta^{\prime}}^{\mathbf{R}}(F)(x)+W_{-\underline{\delta}}^{\mathbf{R}}(F)(x)+\int_{x}^{\infty} W_{-\delta^{\prime}}^{\mathbf{R}}(F)(s) C(x, s) d s\right) \Phi \\
& +\int_{x}^{\infty}\left(W_{-\underline{\delta}}^{\mathbf{R}}(F)+W_{-\delta^{\prime}}^{\mathbf{R}}(F)\right)(s) B_{-\delta^{\prime}}(x, s) d s \cdot \Phi,
\end{aligned}
$$

where, if we denote the first term in the right hand side of (52) by $I_{3}(x) \Phi$, then $K_{3}(x)$ is given by $K_{3}(x)=\int_{x}^{s} I_{3}(s) D_{\Phi}(x, s) d s$. Here, to deduce the last line, we used Lemma $4.4(3), \tilde{W}_{\delta^{\prime}}^{\mathbf{R}} \circ W_{-\delta^{\prime}}^{\mathbf{R}}(C) \sim C$ and the fact that $\left|\int_{x}^{s} C(s, t) B_{-\delta^{\prime}}(x, s) d s\right| \leq$ $c x^{-\delta^{\prime}-1}$. Combining (51) and (53), we can finally deduce that

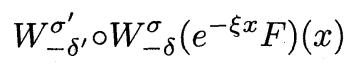

$$
\begin{aligned}
& \sim e^{-\left(\xi+\delta \sigma+\delta^{\prime} \sigma^{\prime}\right) x}\left(F(x)+W_{-\delta}^{\mathbf{R}}(F)(x)+W_{-\delta^{\prime}}^{\mathbf{R}}(F)(x)+W_{-\underline{\delta}}^{\mathbf{R}}(F)(x)\right. \\
& \left.+\int_{x}^{\infty}\left(F(s)+W_{-\delta}^{\mathbf{R}}(F)(s)+W_{-\delta^{\prime}}^{\mathbf{R}}(F)(s)+W_{-\underline{\delta}}^{\mathbf{R}}(F)(s)\right) C(x, s) d s\right) \\
& +x^{-\delta^{\prime}}\left(W_{-\delta^{\prime}}^{\mathbf{R}}(F)(x)+W_{-\underline{\delta}}^{\mathbf{R}}(F)(x)+\int_{x}^{\infty} W_{-\delta^{\prime}}^{\mathbf{R}}(F)(s) C(x, s) d s\right) \Phi \\
& +\int_{x}^{\infty}\left(W_{-\delta^{\prime}}^{\mathbf{R}}(F)+W_{-\underline{\delta}}^{\mathbf{R}}(F)\right)(s) B_{-\delta^{\prime}}(x, s) d s \cdot \Phi .
\end{aligned}
$$

We substitute this formula to (45) and note that

$$
W_{-n}^{\sigma}\left(e^{-\rho x} F\right)(x) \sim e^{-(n \sigma+\rho) x} \sum_{m=0}^{n} c_{m} F^{(m)}(x)
$$

for $x \geq 1$. Then desired result follows from the same argument used in the previous case.

Now let $f \in W_{-}\left(\mathrm{M}_{-s_{\alpha}} H^{1}(\mathbf{R})\right)$, where $s_{\alpha}=\alpha+1 / 2=\underline{n}+\underline{\delta}$ (see (37)). Since the Fourier multiplier $\mathrm{M}_{-s_{\alpha}}$ satisfies the Hörmander condition (cf. [16, $\S 5$ in Chap. 11]), it is bounded on $H^{1}(\mathbf{R})$ (cf. [16, Theorem 4.4 in Chap. 14]). In particular, $F=W_{+}(f)$ belongs to $H^{1}(\mathbf{R})$. We note that

$$
W_{-\gamma}^{\mathbf{R}}(F)^{\sim}(\lambda)=(i \lambda)^{\gamma} F^{\sim}(\lambda)
$$

(cf $\left[13,\left(4.39^{\prime}\right)\right]$ ) and thus, $\mathrm{M}_{s_{\alpha}}^{-1} \circ W_{-\gamma}^{\mathbf{R}}, 0 \leq \gamma \leq s_{\alpha}$, is the the Fourier multiplier corresponding to $(i \lambda)^{\gamma} /(\lambda+i \rho)^{s_{\alpha}}$. Since it satisfies the Hörmander condition, $\mathrm{M}_{s_{\alpha}}^{-1}$ 。 
$W_{-\gamma}^{\mathbf{R}}$ is bounded on $H^{1}(\mathbf{R})$. Hence, each $W_{-\gamma}^{\mathbf{R}}(F)$ also belongs to $H^{1}(\mathbf{R})$. Therefore, the condition that $f \in W_{-}\left(\mathrm{M}_{-s_{\alpha}} H^{1}(\mathbf{R})\right)$ guarantees that for $0 \leq \gamma \leq s_{\alpha}$,

$$
\left\|W_{-\gamma}^{\mathbf{R}}(F)\right\|_{H^{1}(\mathbf{R})}=\left\|M_{\phi}^{\mathbf{R}}\left(W_{-\gamma}^{\mathbf{R}}(F)\right)\right\|_{L^{1}(\mathbf{R})} \leq c\left\|\mathrm{M}_{s_{\alpha}}(F)\right\|_{H^{1}(\mathbf{R})} .
$$

Comparing $M_{\phi}^{\mathbf{R}}\left(W_{-\gamma}^{\mathbf{R}}(F)\right)$ with $M_{\phi} f$, we have the following inequalities.

TheOREM 4.6. Let $\phi$ be as in $\S 3$ and $M \geq 2$. For $f \in W_{-}\left(\mathrm{M}_{-s_{\alpha}} H^{1}(\mathbf{R})\right)$ we put $F=W_{+}(f)$. Then there exist $c_{1}, c_{2}$ such that for all $0 \leq \gamma \leq s_{\alpha}$,

$$
\begin{aligned}
& c_{1}\left\|M_{\phi}^{\mathbf{R}} \circ W_{-\gamma}^{\mathbf{R}}(F)(x)(\operatorname{th} x)^{\gamma}\right\|_{L^{1}(\mathbf{R})} \leq\|f\|_{H_{\phi}^{1}(G)} \\
\leq & c_{2} \sum_{m=0}^{\underline{n}} \sum_{\xi \in \underline{D}}\left\|M_{\phi}^{\mathbf{R}} \circ W_{-(m+\xi)}^{\mathbf{R}}(F)(x)(\operatorname{th} x)^{m+\xi}\right\|_{L^{1}(\mathbf{R})} .
\end{aligned}
$$

Especially,

$$
\begin{aligned}
\|f\|_{H_{\phi}^{1}(G)} & \approx \sum_{m=0}^{\underline{n}} \sum_{\xi \in \underline{D}}\left\|M_{\phi}^{\mathbf{R}} \circ W_{-(m+\xi)}^{\mathbf{R}}(F)(x)(\operatorname{th} x)^{m+\xi}\right\|_{L^{1}(\mathbf{R})} \\
& \leq c \sum_{m=0}^{\underline{n}} \sum_{\xi \in \underline{D}}\left\|W_{-(m+\xi)}^{\mathbf{R}}(F)\right\|_{H^{1}(\mathbf{R})} \\
& \leq c\left\|\mathrm{M}_{s_{\alpha}}(F)\right\|_{H^{1}(\mathbf{R})}
\end{aligned}
$$

and thus, $W_{-}\left(\mathrm{M}_{-s_{\alpha}} H^{1}(\mathbf{R})\right) \subset H_{\phi}^{1}(G / / K)$.

Proof. We denote $\psi_{t}=W_{+}\left(\phi_{t}\right)$, where $\psi_{t}$ is not a dilation of $\psi$, however, as shown in the previous section, $W_{+}\left(\phi_{t}\right)$ has the same properties as a dilation. Therefore, we use this notation to abbrebiate $W_{+}\left(\phi_{t}\right)$. Let $\Phi(x)$ be the same as in the proof of Proposition 4.5. We decompose $f * \phi_{t}$ as

$$
\begin{aligned}
f * \phi_{t} & =W_{-}\left(W_{+}\left(f * \phi_{t}\right)\right)=W_{-}\left(F * \psi_{t}\right) \\
& =W_{-}\left(F * \psi_{t} \cdot \Phi\right)+W_{-}\left(F * \psi_{t} \cdot(1-\Phi)\right)
\end{aligned}
$$

(see (22)). We apply Proposition $4.5(1)$ to $F * \psi_{t} \cdot \Phi$. Then Lemma 4.4 (3) and the same argument used in the proof of Proposition 4.5 yield that

$$
\begin{aligned}
& \sup _{0<t<\infty}\left|W_{-}\left(F * \psi_{t} \cdot \Phi\right)(x)\right| \Delta(x) \\
& \leq c \sum_{m=0}^{\underline{n}} \sum_{\xi \in \underline{D}}\left(x^{m+\xi} M_{\phi}^{\mathbf{R}} \circ W_{-(m+\xi)}^{\mathbf{R}}(F)(x)\right. \\
& \left.\quad+\int_{x}^{\infty} M_{\phi}^{\mathbf{R}} \circ W_{-(m+\xi)}^{\mathbf{R}}(F)(s) A_{m, \xi}^{1}(x, s) d s \Delta(x)\right) .
\end{aligned}
$$


Similarly, applying Proposition $4.5(2)$ to $F * \psi_{t} \cdot(1-\Phi)$, we have

$$
\begin{aligned}
& \sup _{0<t<\infty}\left|W_{-}\left(F * \psi_{t} \cdot(1-\Phi)\right)(x)\right| \Delta(x) \\
& \leq c \sum_{m=0}^{\underline{n}} \sum_{\xi \in \underline{D}}\left(x^{m+\xi} M_{\phi}^{\mathbf{R}} \circ W_{-(m+\xi)}^{\mathbf{R}}(F)(x)\right. \\
& \quad+\int_{x}^{\infty} M_{\phi}^{\mathbf{R}} \circ W_{-(m+\xi)}^{\mathbf{R}}(F)(s) A_{m, \xi}^{2}(x, s) d s \Delta(x) \\
& \left.\quad+x^{m+\xi} \int_{x}^{\infty} M_{\phi}^{\mathbf{R}} \circ W_{-(m+\xi)}^{\mathbf{R}}(F)(s) A_{m, \xi}^{3}(x, s) d s\right) \chi_{[0,1]}(x) \\
& +c \sum_{m=0}^{\underline{n}} \sum_{\xi \in \underline{D}}\left(M_{\phi}^{\mathbf{R}} \circ W_{-(m+\xi)}^{\mathbf{R}}(F)(x)\right. \\
& \quad+\int_{x}^{\infty} M_{\phi}^{\left.\mathbf{R} \circ W_{-(m+\xi)}^{\mathbf{R}}(F)(s) A_{m, \xi}^{4}(x, s) d s\right) \chi_{[1, \infty)}(x) .}
\end{aligned}
$$

We recall that $A_{m, \xi}^{j}(x, s), j=3,4$, satisfy (40), and for $j=1,2$ and $0<x \leq s \leq 1$, $A_{m, \xi}^{j}(x, s) \Delta(x) \leq c x^{m+\xi-1}$ (see (39), (4)) and thus,

$$
\int_{0}^{s} A_{m, \xi}^{j}(x, s) \Delta(x) d x \leq c \int_{0}^{s} x^{m+\xi-1} d x \leq c s^{m+\xi}, \quad 0<s \leq 1 .
$$

Therefore, we can deduce that

$$
\begin{aligned}
\|f\|_{H^{1}(G)}=\left\|M_{\phi} f\right\|_{1} & =\int_{0}^{\infty} \sup _{0<t<\infty}\left|W_{-}\left(F * \psi_{t}\right)(x)\right| \Delta(x) d x \\
& \leq \sum_{m=0}^{\underline{n}} \sum_{\xi \in \underline{D}}\left\|M_{\phi}^{\mathbf{R}} \circ W_{-(m+\xi)}^{\mathbf{R}}(F)(\operatorname{th} x)^{m+\xi}\right\|_{L^{1}(\mathbf{R})} .
\end{aligned}
$$

As for the first inequality in the theorem we recall that

$$
\begin{aligned}
F * \psi_{t}(x) & =W_{+}\left(f * \phi_{t}\right)(x) \\
& =e^{\rho x} W_{\alpha-\beta}^{1} \circ W_{\beta+1 / 2}^{2}\left(f * \phi_{t}\right)(x) \\
& =c e^{\rho x} \int_{x}^{\infty} f * \phi_{t}(s) A(x, s) d s
\end{aligned}
$$

and for $0 \leq \gamma \leq s_{\alpha}$ and $0<x \leq s$,

$$
\left|W_{-\gamma}^{\mathbf{R}} A(x, s)\right| \leq c e^{(\rho-2) s}(\operatorname{sh} 2 s)(\text { th } s)^{2 \alpha-1-\gamma} \leq c e^{\rho s}(\operatorname{th} s)^{2 \alpha-\gamma}
$$


(see (15), (17)). Hence,

$$
\left|W_{-\gamma}^{\mathbf{R}}(F) * \psi_{t}(x)\right|=\left|W_{-\gamma}^{\mathbf{R}}\left(F * \psi_{t}\right)(x)\right| \leq c e^{\rho x} \int_{x}^{\infty}\left|f * \phi_{t}(s)\right| e^{\rho s}(\text { th } s)^{2 \alpha-\gamma} d s .
$$

We take the supremum over $0<t<\infty$. Since $2 \alpha-\gamma \geq 2 \alpha-s_{\alpha}=\alpha-1 / 2>-1$, we can deduce that,

$$
\begin{aligned}
& \int_{0}^{\infty} M_{\phi}^{\mathbf{R}} \circ W_{-\gamma}^{\mathbf{R}}(F)(x)(\operatorname{th} x)^{\gamma} d x \\
\leq & c \int_{0}^{\infty} e^{\rho x}\left(\int_{x}^{\infty} M_{\phi} f(s) e^{\rho s}(\operatorname{th} s)^{2 \alpha-\gamma} d s\right)(\operatorname{th} x)^{\gamma} d x \\
\leq & c \int_{0}^{\infty} M_{\phi} f(s) e^{2 \rho s}(\operatorname{th} s)^{2 \alpha+1} d s \sim\left\|M_{\phi} f\right\|_{1}=\|f\|_{H^{1}(G)} .
\end{aligned}
$$

This completes the proof of the theorem.

REMARK 4.7. (1) Since

$$
C(-(\lambda+i \rho)) \sim(1+|\lambda|)^{-(\alpha+1 / 2)}, \quad \lambda \in \mathbf{R}
$$

(see [3, Theorem 2]), Theorem 4.6 means that

$$
W_{-}\left(\mathrm{M}_{C_{\rho}}\left(H^{1}(\mathbf{R})\right)\right)=W_{-}\left(\mathrm{M}_{-s_{\alpha}} H^{1}(\mathbf{R})\right) \subset H_{\phi}^{1}(G / / K) .
$$

Theorem A in $\S 1$ follows from this relation and the one in Corollary 4.3.

(2) Since $(\operatorname{th} x)^{m+\xi}$ is bounded, it is easy to see that $M_{\phi}^{\mathbf{R}}$ in Theorem 4.6 can be replaced by $\mathrm{M}_{\ell}, \ell \in \mathrm{A}_{N, 2 M}$ (see Definitions 8.4 and 8.5, and cf. Theorem 8.6). Especially, $H_{\phi}^{1}(G / / K)$ does not depend on an individual $\phi$. We shall skip writing $\phi$ and denote simply as

$$
H^{1}(G / / K)=H_{\phi}^{1}(G / / K), \quad\|f\|_{H^{1}(G)}=\|f\|_{H_{\phi}^{1}(G)} .
$$

We keep the notations in the proof of Theorem 4.6 and we shall suppose that $t \geq 1$. Since we can transfer the Fourier multiplier $W_{-\gamma}^{\mathbf{R}}$ as

$$
W_{-\gamma}^{\mathbf{R}}\left(F * \psi_{t}\right)=W_{-\gamma}^{\mathbf{R}}(F) * \psi_{t}=F * W_{-\gamma}^{\mathbf{R}}\left(\psi_{t}\right),
$$

it follows from the proof of Theorem 4.6 that for each $C \geq 0$,

$$
\begin{aligned}
& \int_{0}^{\infty} \sup _{t \geq 1}\left|f * \phi_{t}(x)\right| \Delta(x) d x \\
\leq & c \sum_{m=0}^{\underline{n}} \sum_{\xi \in \underline{D}} \int_{0}^{\infty} \sup _{t \geq 1}\left|F * W_{-(m+\xi)}^{\mathbf{R}}\left(\psi_{t}\right)(x)\right| d x
\end{aligned}
$$




$$
\begin{aligned}
& \leq c \sum_{m, \xi, m+\xi \neq 0} \int_{0}^{\infty} \sup _{t \geq 1}\left|F *\left(W_{-(m+\xi)}^{\mathbf{R}}\left(\psi_{t}\right)-C \psi_{t}\right)(x)\right| d x \\
& \quad+d \int_{0}^{\infty} \sup _{t \geq 1}\left|F * \psi_{t}(x)\right| d x
\end{aligned}
$$

where $d=c(1+(4 \underline{n}-1) C)$. We note that

$$
\begin{aligned}
W_{-\gamma}^{\mathbf{R}}\left(\psi_{t}\right)^{\sim}(\lambda) & =W_{+}\left(\phi_{t}\right)^{\sim}(\lambda)(i \lambda)^{\gamma} \\
& =\hat{\phi}_{t}(\lambda+i \rho)(i t \lambda)^{\gamma} \cdot t^{-\gamma}
\end{aligned}
$$

Since $t \geq 1$, Lemma 3.5 implies that each $W_{-\gamma}^{\mathbf{R}}\left(\psi_{t}\right)^{\sim}(\lambda), 0 \leq \gamma \leq s_{\alpha}=\alpha+1 / 2$, belongs to $\mathbf{A}_{N, 2 M}$ (see Definition 8.4). Moreover, if $\gamma>0$, then $W_{-\gamma}^{\mathbf{R}}\left(\psi_{t}\right) \sim(\lambda) \rightarrow 0$ as $|t \lambda| \rightarrow 0$, because it has the term $(t \lambda)^{\gamma}$ in $(55)$. Since $\left(\psi_{t}\right)^{\sim}(\lambda)=\hat{\phi}_{t}(\lambda+i \rho)$ satisfies Lemma $3.6(2)$, we may suppose that for a sufficiently large $C>0$, each $\ell_{m+\xi}(t, \lambda)=W_{-(m+\xi)}^{\mathbf{R}}\left(\psi_{t}\right)^{\sim}(\lambda)-C \psi_{t}^{\sim}(\lambda)$ also belongs to $\mathrm{A}_{N, 2 M}$ and satisfies the assumption (90) in Theorem 8.6. Hence, Proposition 3.7 and Theorem 8.6 yield that, if $M \geq 2$ in (24), then

$$
\begin{aligned}
& \int_{0}^{\infty} \sup _{t \geq 1}\left|f * \phi_{t}(x)\right| \Delta(x) d x \\
\leq & c \int_{0}^{\infty}\left(\sum_{m, \xi, m+\xi \neq 0} \sup _{t \geq 1}\left|\left(\mathrm{M}_{\ell_{m+\xi}(t, \cdot)} F\right)(x)\right|+M_{\phi}^{\mathbf{R}} F(x)\right) d x \\
\leq & c \sum_{m, \xi, m+\xi \neq 0}\left\|M_{\ell_{m+\xi}} F\right\|_{L^{1}(\mathbf{R})}+c\left\|M_{\phi}^{\mathbf{R}} F\right\|_{L^{1}(\mathbf{R})} \leq c\|F\|_{H^{1}(\mathbf{R})}
\end{aligned}
$$

(see $\S 8 . \mathrm{B}$ for the definitions of $\mathrm{M}_{\ell_{m+\xi}(t, \cdot)}$ and $M_{\ell_{m+\xi}}$ ). We here define a truncated maximal operator $M_{\phi}^{\text {loc }}$ on $G$ as follows.

Definition 4.8. For $f \in L_{\text {loc }}^{1}(G / / K)$,

$$
\left(M_{\phi}^{\mathrm{loc}} f\right)(g)=\sup _{0<t<1}\left|\left(f * \phi_{t}\right)(g)\right|, \quad g \in G .
$$

Then (56) implies that $\|f\|_{H^{1}(G)} \leq\left\|M_{\phi}^{\text {loc }} f\right\|_{1}+c\|F\|_{H^{1}(\mathbf{R})}$. Hence, Corollary 4.3 yields the following.

Theorem 4.9. Let $M \geq 2$. For $f \in H^{1}(G / / K)$ we put $F=W_{+}(f)$. Then

$$
\|f\|_{H^{1}(G)} \approx\left\|M_{\phi}^{\mathrm{loc}} f\right\|_{1}+\|F\|_{H^{1}(\mathbf{R})} .
$$




\section{Atomic Hardy spaces on $G$}

In this section we shall introduce some $K$-bi-invariant atoms on $G$ and define the corresponding atomic Hardy spaces on $G$, on which the radial maximal operator $M_{\phi}$ is bounded to $L^{1}(G)$.

For $x \in \mathbf{R}$ and $r>0$, let $R(x, r)$ denote the interval on $\mathbf{R}$ centered at $x$ with radius $r$ and $|R(x, r)|$ its volume with respect to $\Delta(x) d x$ :

$$
R(x, r)=[x-r, x+r] \text { and }|R(x, r)|=\int_{x-r}^{x+r} \Delta(s) d s .
$$

For $x \in G$ and $r>0$, we also denote the annulus $\{y \in G ;|\sigma(x)-\sigma(y)| \leq r\}=$ $K\left\{a_{s} ; s \in R(\sigma(x), r)\right\} K$ on $G$ by the same notation $R(x, r)$. Clearly, $R(x, r)=$ $R\left(a_{\sigma(x)}, r\right)$ for $x \in G$. We put $B(r)=R(e, r)$ if $x=e$. Obviously, if $\sigma(x) \geq r$, then the volume $|R(x, r)|$ of $R(x, r)$ with respect to $d g$ coincides with $|R(\sigma(x), r)|$ (see (57)) . Moreover, if $\sigma(x) \leq r$, then $R(x, r)=B(\sigma(x)+r)$.

Definition 5.1. We say that a $K$-bi-invariant function $a$ on $G$ is a $(1, \infty, 0)$ atom provided that there exist $x \in G$ and $0<r \leq \sigma(x)$ such that

$$
\begin{gathered}
\operatorname{supp}(a) \subset R(x, r) \\
\|a\|_{\infty} \leq|R(x, r)|^{-1} \\
\int_{G} a(g) d g=0 .
\end{gathered}
$$

For $\epsilon \geq 0$, we say that $a$ is a $(1, \infty, 0, \epsilon)$-atom if we replace $(i i)$ by

$$
\|a\|_{\infty} \leq|R(x, r)|^{-1}(1+r)^{-\epsilon},
$$

and a $(1, \infty,+)$-atom if we replace $(i i i)$ by

$$
(i i i)_{+} \quad \int_{G} a(g) d g=0 \text { if } r<1 .
$$

Moreover, if $x=0$, we call $a$ a centered atom.

We introduce atomic Hardy spaces on $G$ as follows.

Definition 5.2. We define

$$
H_{\infty, 0}^{1}(G / / K)=\left\{f=\sum_{i} \lambda_{i} a_{i} ; a_{i} \text { is a }(1, \infty, 0) \text {-atom on } G \text { and } \sum_{i}\left|\lambda_{i}\right|<\infty\right\}
$$

and $\|f\|_{H_{\infty, 0}^{1}(G)}=\inf \sum_{i}\left|\lambda_{i}\right|$, where the infimum is taken over all such representations $f \stackrel{\infty, 0}{=} \sum_{i} \lambda_{i} a_{i}$. Similarly, replacing $(1, \infty, 0)$-atoms by $(1, \infty, 0, \epsilon)$-atoms and $(1, \infty,+)$-atoms, we define $H_{\infty, 0}^{1, \epsilon}(G / / K)$ and $H_{\infty, 0}^{1,+}(G / / K)$ respectively. 
Definition 5.3. We define the small Hardy space $h_{\infty, 0}^{1}(G / / K)$ on $G$ by restricting $(1, \infty, 0)$-atoms in the above definition of $H_{\infty, 0}^{1}(G / / K)$ to ones with radius $\leq 1$.

Clearly, we have

$$
h_{\infty, 0}^{1}(G / / K) \subset H_{\infty, 0}^{1, \epsilon}(G / / K) \subset H_{\infty, 0}^{1}(G / / K) \subset H_{\infty, 0}^{1,+}(G / / K)
$$

In what follows we shall characterize the difference between $h_{\infty, 0}^{1}(G / / K)$ and $H_{\infty, 0}^{1,+}(G / / K)$, and then we shall obtain a relation between $H_{\infty, 0}^{1,+}(G / / K) \cap$ $W_{-}\left(H^{1}(\mathbf{R})\right)$ and $H_{\phi}^{1}(G / / K)$. For $x_{0} \in G$ and $r>0$, let $\chi_{x_{0}, r}$ denote the characteristic function of $R\left(x_{0}, r\right)$ on $G$. For $x, y \in G$, we define

$$
I_{x_{0}}(x, r, y)=\int_{K} \chi_{x_{0}, r}\left(x^{-1} k y\right) d k .
$$

Then $I_{x_{0}}(x, r, y)$ is $K$-bi-invariant with respect to $x, y$ and, as a function of $y$, it is supported on $R\left(x, \sigma\left(x_{0}\right)+r\right)$, where $R\left(x, \sigma\left(x_{0}\right)+r\right)=B\left(\sigma(x)+\sigma\left(x_{0}\right)+r\right)$ if $\sigma(x) \leq \sigma\left(x_{0}\right)+r$. For simplicity, when $x_{0}=e$, we skip writing the suffices $x_{0}=e$ :

$$
\chi_{r}=\chi_{e, r}, \quad I(x, r, y)=I_{e}(x, r, y) .
$$

Lemma 5.4. Let $x, y \in G$ and $\sigma(x)>r$. Then

(1) $I(x, r, y) \leq I(x, r, x)$,

(2) $|B(r)||R(x, r)|^{-1} \leq I(x, r, x) \leq|B(2 r)||R(x, r)|^{-1}$,

(3) $I_{x_{0}}(x, r, y) \leq|B(2 r)||R(x, r)|^{-1}$ if $r \geq 1, \sigma(x) \geq 1, \sigma\left(x_{0}\right) \geq r+1$.

Proof. We regard $I(x, r, y)$ as a function on $\mathbf{R}_{+} \times \mathbf{R}_{+}$. For a fixed $x$, as a function of $y$, it is supported on $|x-y| \leq r$ and $I(x, r, y)$ is increasing on $x-r \leq y \leq x$. Hence (1) is obvious. As for (2), let $f$ be an arbitrary function in $L^{\infty}(G / / K)$. Then

$$
\begin{aligned}
\chi_{r} * f(x) & =\int_{G} \chi_{r}\left(x g^{-1}\right) f(g) d g \\
& =\int_{x-r}^{x+r} I(x, r, s) f(s) \Delta(s) d s .
\end{aligned}
$$

Therefore, letting $f \equiv 1$, we see from (1) that

$$
\chi_{r} * f(x)=|B(r)| \leq I(x, r, x)|R(x, r)| .
$$

Similarly, letting $f$ be the characteristic function of $R(x,|x-y|)$, we see that

$$
\chi_{r} * f(x) \geq I(x, r, y)|R(x,|x-y|)|
$$


and thus, $I(x, r, y) \leq\left\|\chi_{r}\right\|_{1}\|f\|_{\infty}|R(x,|x-y|)|^{-1} \leq|B(r)||R(x,|x-y|)|^{-1}$. Hence

$$
\begin{aligned}
I(x, r, x) & \leq I(x-r, 2 r, x-r) \\
& \leq I(x, 2 r, x-r) \\
& \leq|B(2 r)||R(x, r)|^{-1}
\end{aligned}
$$

As for (3), we may suppose that $x_{0}, x, y \in A_{+} \cong \mathbf{R}_{+}$and we use the kernel form of the product of spherical functions:

$$
\varphi_{\lambda}(x) \varphi_{\lambda}(y)=\int_{0}^{\infty} K(x, y, z) \varphi_{\lambda}(z) \Delta(z) d z
$$

(see $[4,(4.2)]$ ). Since $x \geq 1, x_{0}-r \geq 1$, it follows that

$$
\begin{aligned}
I_{x_{0}}(x, r, y) & =\int_{x_{0}-r}^{x_{0}+r} K(x, y, z) \Delta(z) d z \\
& \leq c e^{-\rho x} e^{-\rho\left(x_{0}+x-r\right)} \int_{x_{0}-r}^{x_{0}+r} e^{\rho z} d z \\
& \leq c e^{-2 \rho x} e^{2 \rho r} \sim|B(2 r)||R(x, r)|^{-1}
\end{aligned}
$$

We set

$$
\theta(g)=|B(1)|^{-1} \chi_{1}(g), \quad g \in G,
$$

where $\chi_{1}$ is the characteristic function of $B(1)$, and for each (not necessary $K$-biinvariant) function $f$ on $G$, we define a $K$-bi-invariant function $f_{x}^{b}, x \in G$, as

$$
f_{x}^{b}(g)=\int_{K} \int_{K} f\left(x^{-1} k g k^{\prime}\right) d k d k^{\prime} \quad(g \in G) .
$$

Proposition 5.5. For $f \in H_{\infty, 0}^{1,+}(G / / K)$ there exist $f_{0} \in h_{\infty, 0}^{1}(G / / K)$ and $x_{i} \in G, \lambda_{i} \in \mathbf{R}$ such that

$$
f=f_{0}+\sum_{i} \lambda_{i} \theta_{x_{i}}^{b}
$$

where $\left\|f_{0}\right\|_{h_{\infty, 0}^{1}(G)}$ and $\sum_{i}\left|\lambda_{i}\right|$ are both bounded by $\|f\|_{H_{\infty, 0}^{1,+}(G)}$.

Proof. By Definitions 5.2 and 5.3, it is enough to obtain the above decomposition for a $(1, \infty,+)$-atom $a$ on $G$ with radius $r \geq 1$. Let $B\left(a_{\sigma(x)}, r\right), x \in \mathbf{R}_{+}$, denote the support of $a$. We identify $K$-bi-invariant functions on $G$ with functions on $\mathbf{R}_{+}$, so $a$ is supported on $R(x, r)$. Without loss of generality we may suppose 
that $x, r \in \mathbf{N}$. Actually, since $r \geq 1,|R(x, r)| \sim|R([x],[r])|$ uniformly on $x \geq 0$ and $r>1$. We decompose $R(x, r)$ as

$$
R(x, r) \subset \bigcup_{k=0}^{r} I_{k}, \quad I_{k}=R(x-r+2 k, 1),
$$

and set $a_{k}=\left.\left|I_{k}\right|^{-1}|R(x, r)| \cdot a\right|_{I_{k}}$. Then, $a_{k}$ is supported on $I_{k},\left\|a_{k}\right\|_{\infty} \leq\left|I_{k}\right|^{-1}$ and

$$
a=\sum_{k=0}^{2 r} \mu_{k} a_{k}, \quad \mu_{k}=\left|I_{k}\right||R(x, r)|^{-1} .
$$

Here $\sum_{k} \mu_{k} \sim 1$. Let $\theta_{k}=\theta_{a_{x-r+k}}^{b}(0 \leq k \leq r)$, where $a_{x-r+k}$ is the element in $A_{+}$ corresponding to $x-r+k \in \mathbf{R}_{+}$(see (3), (62), (63)). Clearly, $\theta_{k}$ is supported on $I_{k}, \int_{G} \theta_{k}(g) d g=\int_{G} \theta(g) d g=1$, and $\left\|\theta_{k}\right\|_{\infty} \leq\left|I_{k}\right|^{-1}$ by Lemma 5.4 (1), (2). Let $m_{k}=\int_{G} a_{k}(g) d g$ and $b_{k}=\left(a_{k}-m_{k} \theta_{k}\right) / 2$. Then $\left|m_{k}\right| \leq 1, b_{k}$ is supported on $I_{k}$, $\left\|b_{k}\right\|_{\infty} \leq\left|I_{k}\right|^{-1}$, and $\int_{G} b_{k}(g) d g=0$. This means that $b_{k}$ is a $(1, \infty, 0)$-atom with radius 1 . Therefore, letting

$$
a=\sum_{k=0}^{2 r} 2 \mu_{k} b_{k}+\sum_{k=0}^{2 r} \mu_{k} m_{k} \theta_{k}
$$

we have the desired decomposition of a $(1, \infty,+)$-atom $a$ with $r \geq 1$.

We set

$$
\langle\theta\rangle=\left\{\sum_{i} \lambda_{i} \theta_{x_{i}}^{b} ; \sum_{i}\left|\lambda_{i}\right|<\infty, x_{i} \in G\right\} .
$$

Then Proposition 5.5 means that

$$
H_{\infty, 0}^{1,+}(G / / K)=h_{\infty, 0}^{1}(G / / K)+\langle\theta\rangle .
$$

Next we shall restrict our attention to centered atoms $a$ and consider a linear combination of their averaged translations $a_{x}^{b}, x \in G$ (see (63)).

Definition 5.6. We define

$$
\begin{array}{r}
\mathrm{H}_{\infty, 0}^{1}(G / / K)=\left\{f=\sum_{i} \lambda_{i} a_{i, x_{i}}^{b} ; a_{i} \text { is a }(1, \infty, 0) \text {-centered atom on } G,\right. \\
\left.x_{i} \in G, \text { and } \sum_{i}\left|\lambda_{i}\right|<\infty\right\}
\end{array}
$$

and $\|f\|_{\mathrm{H}_{\infty, 0}^{1}(G)}=\inf \sum_{i}\left|\lambda_{i}\right|$, where the infimum is taken over all such representations $f=\sum_{i} \lambda_{i} a_{i, x_{i}}^{b}$. Similarly, replacing centered $(1, \infty, 0)$-atoms $a_{i}$ by centered 
$(1, \infty, 0, \epsilon)$-atoms, centered $(1, \infty,+)$-atoms and centered atoms with radius $\leq 1$ respectively, we define $\mathrm{H}_{\infty, 0}^{1, \epsilon}(G / / K), \mathrm{H}_{\infty, 0}^{1,+}(G / / K), \mathrm{h}_{\infty, 0}^{1}(G / / K)$.

In this definition each atom $a_{i}$ is $K$-bi-invariant (see Definition 5.1). We shall consider non- $K$-bi-invariant cases. Let $a_{i}$ be a centered function on $G$ satisfying (58) to (60), not necessary $K$-bi-invariant. Even if $a_{i}$ is not $K$-bi-invariant, $a_{i, x_{i}}^{b}$ is $K$-bi-invariant. Hence, by using such $a_{i}$, let $\mathrm{H}_{\infty, 0}^{1}(G / / K)^{\natural}$ denote the space of all $f=\sum_{i} \lambda_{i} a_{i, x_{i}}^{b}$ with $x_{i} \in G$ and $\sum_{i}\left|\lambda_{i}\right|<\infty$. Similarly, we define $H_{\infty, 0}^{1, \epsilon}(G / / K)^{\natural}$, $\mathrm{H}_{\infty, 0}^{1,+}(G / / K)^{\natural}$ and $\mathrm{h}_{\infty, 0}^{1}(G / / K)^{\natural}$ respectively as in Definition 5.6.

Proposition 5.7. Let $\epsilon>0$. We have the following inclusions:

$$
\begin{array}{cccc}
\mathrm{h}_{\infty, 0}^{1}(G / / K)^{\natural} & \subset \mathrm{H}_{\infty, 0}^{1, \epsilon}(G / / K)^{\natural} & \subset \mathrm{H}_{\infty, 0}^{1}(G / / K)^{\natural} \subset \mathrm{H}_{\infty, 0}^{1,+}(G / / K)^{\natural} \\
\| & \cup & \cup & \cup \\
h_{\infty, 0}^{1}(G / / K) & \subset H_{\infty, 0}^{1, \epsilon}(G / / K) & \subset H_{\infty, 0}^{1}(G / / K) & \subset H_{\infty, 0}^{1,+}(G / / K) \\
\cup & \cup & \cup & \cup \\
\mathrm{h}_{\infty, 0}^{1}(G / / K) & \subset \mathrm{H}_{\infty, 0}^{1, \epsilon}(G / / K) & \subset \mathrm{H}_{\infty, 0}^{1}(G / / K) & \subset \mathrm{H}_{\infty, 0}^{1,+}(G / / K) .
\end{array}
$$

Proof. The horizontal inclusions are clear from the definitions. We first prove that each space in the first line contains the bottom one in the second line.

LemMA 5.8. Let a be a K-bi-invariant function on $G$ supported on $R(x, r)$ and $\|a\|_{\infty} \leq|R(x, r)|^{-1}$. Let $r_{0}=2 r$ if $r \leq 1$ and $r_{0}=r+1$ if $r>1$. Then there exists a constant $c>0$ such that

$$
\left\|a / I\left(x, r_{0}, \cdot\right)\right\|_{\infty} \leq c|B(r)|^{-1}
$$

where $c$ is independent of $x, r$.

Proof. If $r \leq 1$, then Lemma 5.4 implies that for $|x-y|<r$,

$$
\begin{aligned}
I\left(x, r_{0}, y\right) & \geq I\left(x, r_{0}, x-r\right) \\
& \geq I(x-r, r, x-r) \\
& \geq|B(r)||R(x-r, r)|^{-1} \\
& \geq|B(r)||R(x, r)|^{-1} .
\end{aligned}
$$

Similarly, if $r>1$, then $I\left(x, r_{0}, y\right) \geq I(x-r, 1, x-r) \geq|B(1)||R(x-r, 1)|^{-1} \sim$ $e^{-2 \rho(x-r)} \geq|B(r)||R(x, r)|^{-1}$. Thereby, $\left\|a / I\left(x, r_{0}, \cdot\right)\right\|_{\infty} \leq c|B(r)|^{-1}$.

We recall the relation $H_{\infty, 0}^{1, \sharp}(G)=H_{\infty, 0}^{1, b}(G)$ obtained in [9, Theorem 5.5]. Here $H_{\infty, 0}^{1, b}(G)$ is nothing but $H_{\infty, 0}^{1,+}(G / / K)^{\sharp}$ in this paper and $H_{\infty, 0}^{1, \sharp}(G)$ contains $H_{\infty, 0}^{1,+}(G / / K)$ by Lemma 5.8. Then, it is easy to see that each space in the first 
line contains the bottom one in the second line. Furthermore, since $I(x, r, y) \leq$ $c|R(x, r)|^{-1}$ if $r \leq 1$ by Lemma 5.4 , it follows that $h_{\infty, 0}^{1}(G / / K)^{\natural} \subset h_{\infty, 0}^{1}(G / / K)$.

Next we shall prove that each space in the second line contains the bottom one in the third line.

$\mathrm{H}_{\infty, 0}^{1}(G / / K) \subset H_{\infty, 0}^{1}(G / / K)$ : Let $a$ be a centered $(1, \infty, 0)$-atom on $G$ supported on $B(r)$ and $x \in G$. Then $a_{x}^{b}$ is supported on $R(x, r), \int_{G} a_{x}^{b}(g) d g=$ $\int_{G} a(g) d g=0$, and $\left\|a_{x}^{b}\right\|_{\infty} \leq|R(x, r)|^{-1}|B(2 r)||B(r)|^{-1}$ by (61), (63) and Lemma 5.4 (2). Therefore, if $r \leq 1$, then $\left\|a_{x}^{b}\right\|_{\infty} \leq c|R(x, r)|^{-1}$. This means that $c^{-1} a_{x}^{b}$ is a $(1, \infty, 0)$-atom on $G$. Let $r>1$ and $\sigma(x) \leq 2$. We may regard that $a_{x}^{b}$ is supported on $B(\sigma(x)+r)$ and we note that $\left\|a_{x}^{b}\right\|_{\infty} \leq|B(r)|^{-1} \leq c|R(\sigma(x)+r)|^{-1}$. Therefore, $c^{-1} a_{x}^{b}$ is a $(1, \infty, 0)$-atom on $G$. Let $r>1$ and $\sigma(x)>2$. In this case, since $a$ is centered, $a$ has an $L^{1}$ non-increasing denominator $|B(r)|^{-1} \chi_{B(r)}(x)$ (see [7, 4.4]). Then $a$ can be decomposed as $a=\sum_{i} \lambda_{i} a_{i}$, where $\sum_{i}\left|\lambda_{i}\right| \sim 1$ and each $a_{i}$ is a $(1, \infty, 0)$-atom supported on $R\left(x_{i}, 1\right), x_{i} \in \mathbf{N}$ and $0<x_{i} \leq r$ (see the proof of [7, Theorem 4.5]). Hence $a_{i, x}^{b}$ is supported on $R\left(x, x_{i}+1\right.$ ) and $\left\|a_{i, x}^{b}\right\|_{\infty} \leq\left|R\left(x_{i}, 1\right)\right|^{-1}|B(2)||R(x, 1)|^{-1} \leq c\left|R\left(x, x_{i}+1\right)\right|^{-1}$ by $(61)$, (63) and Lemma 5.4 (3). Therefore, each $c^{-1} a_{i, x_{i}}^{b}$ is a $(1, \infty, 0)$-atom on $G$. These observations imply that $a \in H_{\infty, 0}^{1}(G / / K)$ and its norm is bounded by a constant independent of $a$. Hence the desired inclusion follows.

$\mathrm{h}_{\infty, 0}^{1}(G / / K) \subset h_{\infty, 0}^{1}(G / / K)$ : This is clear from the case of $r \leq 1$ in the above argument.

$\mathrm{H}_{\infty, 0}^{1, \epsilon}(G / / K) \subset H_{\infty, 0}^{1, \epsilon}(G / / K)$ : Let $r>1$ and $a$ be a centered $(1, \infty, 0, \epsilon)$-atom on $G$. We repeat the above argument. Since $\|a\|_{\infty}$ has an extra decay $r^{-\epsilon},\left\|a_{x}^{b}\right\|_{\infty}$ in $\sigma(x) \leq 2$ and $\left\|a_{i, x}^{b}\right\|_{\infty}$ in $\sigma(x)>2$ also have the same extra decay. Since $r^{-\epsilon} \leq c(\sigma(x)+r)^{-\epsilon}$ in $\sigma(x) \leq 2$ and $r^{-\epsilon} \leq x_{i}^{-\epsilon} \leq c\left(x_{i}+1\right)^{-\epsilon}$ in $\sigma(x)>2$, it follows that $a_{x}^{b}$ and $a_{i, x}^{b}$ are $(1, \infty, 0, \epsilon)$-atoms on $G$ up to a constant multiplication. Hence the desired inclusion follows.

$\mathrm{H}_{\infty, 0}^{1,+}(G / / K) \subset H_{\infty, 0}^{1,+}(G / / K)$ : Let $r>1$ and $a$ be a centered $(1, \infty,+)$-atom on $G$. Then Proposition 5.5 means that $a$ is decomposed as $a=\sum_{i} \lambda_{i} a_{i}$, where $\sum_{i}\left|\lambda_{i}\right| \sim 1$ and each $a_{i}$ is a $(1, \infty,+)$-atom supported on $R\left(x_{i}, 1\right), x_{i} \in \mathbf{N}$ and $\left|x_{i}\right| \leq r$. As in the previous case, since $R\left(x_{i}, 1\right)$ has the radius 1 , each $a_{i, x}, x \in G$, is also a $(1, \infty,+)$-atom on $G$ up to constant multiplication. Hence the desired inclusion follows.

Let $\phi$ be the $K$-bi-invariant function on $G$ introduced in $\S 3$ and $M_{\phi}^{\text {loc }}$ the truncated maximal operator in Definition 4.8. Since $H_{\infty, 0}^{1, b}(G)$ in [9] coincides with $\mathrm{H}_{\infty, 0}^{1,+}(G / / K)^{\sharp}$, it follows from [9, Theorem 5.3] that 
Proposition 5.9. Let $M \geq 2$. $M_{\phi}$ is bounded from $\mathrm{H}_{\infty, 0}^{1,+}(G / / K)^{\sharp} \cap$ $W_{-}\left(H^{1}(\mathbf{R})\right)$ to $L^{1}(G / / K)$, that is, there exists a constant $c>0$ such that

$$
\left\|M_{\phi} f\right\|_{1} \leq c\left(\|f\|_{\mathrm{H}_{\infty, 0}^{1,+}(G)^{\sharp}}+\left\|W_{+}(f)\right\|_{H^{1}(\mathbf{R})}\right)
$$

for all $f \in \mathrm{H}_{\infty, 0}^{1,+}(G / / K)^{\sharp} \cap W_{-}\left(H^{1}(\mathbf{R})\right)$ and thus,

$$
\mathrm{H}_{\infty, 0}^{1,+}(G / / K)^{\sharp} \cap W_{-}\left(H^{1}(\mathbf{R})\right) \subset H^{1}(G / / K) .
$$

Theorem 5.10. Let $M \geq 2 . M_{\phi}$ is bounded from $H_{\infty, 0}^{1,1}(G / / K)$ to $L^{1}(G / / K)$, that is, there exists a constant $c>0$ such that

$$
\left\|M_{\phi} f\right\|_{1} \leq c\|f\|_{H_{\infty, 0}^{1,1}(G)}
$$

for all $f \in H_{\infty, 0}^{1,1}(G / / K)$ and thus,

$$
H_{\infty, 0}^{1,1}(G / / K) \subset H^{1}(G / / K)
$$

Proof. Since $H_{\infty, 0}^{1,1}(G / / K) \subset \mathrm{H}_{\infty, 0}^{1,+}(G / / K)^{\sharp}$ (see Proposition 5.7), it is enough to prove that $H_{\infty, 0}^{1,1}(G / / K) \subset W_{-}\left(H^{1}(\mathbf{R})\right)$ (see Proposition 5.9). We shall prove that, for all $(1, \infty, 0,1)$-atoms $a$ on $G,\left\|W_{+}(a)\right\|_{H^{1}(\mathbf{R})} \leq c$, where $c$ is independent of $a$.

Lemma 5.11. Let $\gamma \in \mathbf{R}$ and $\sigma>0$. If $F$ is supported on $R\left(x_{0}, r\right)$ and smooth if $\gamma<0$, then $W_{\gamma}^{\sigma}(F)(|x|)$ is also supported on $R\left(x_{0}, r\right)$.

Proof. When $\gamma=-n, n \in \mathbf{N}, W_{-n}^{\sigma}$ is a differential operator $(d / d \operatorname{ch} \sigma x)^{n}$ and thus, the desired result is obvious. Hence, it is enough to consider the case of $\gamma>0$. We denote $F_{0}(x)=F\left(x+x_{0}\right)$. Then it follows from (14) that

$$
\begin{aligned}
& W_{-\delta}^{\sigma}(F)(|x|) \\
= & c \int_{|x|}^{\infty} F(s)(\operatorname{ch} \sigma s-\operatorname{ch} \sigma x)^{\gamma-1} \operatorname{sh} s d s \\
= & c \int_{\left|x-x_{0}\right|}^{\infty} F_{0}(s)\left(\operatorname{ch} \sigma\left(s+x_{0}\right)-\operatorname{ch} \sigma x\right)^{\gamma-1} \operatorname{sh}\left(s+x_{0}\right) d s \\
= & c \int_{\left|x-x_{0}\right|}^{\infty} F_{0}(s)\left(\left(\operatorname{ch} \sigma s-\operatorname{ch} \sigma\left(x-x_{0}\right)\right) \operatorname{ch} x_{0}+\left(\operatorname{sh} \sigma s-\operatorname{sh} \sigma\left(x-x_{0}\right)\right) \operatorname{sh} x_{0}\right)^{\gamma-1} \\
= & G_{0}\left(x-x_{0}\right) \operatorname{ch} x_{0}+G_{1}\left(x-x_{0}\right) \operatorname{sh} x_{0},
\end{aligned}
$$


where

$$
G_{0}(x)=c \int_{|x|}^{\infty} F_{0}(s)\left((\operatorname{ch} \sigma s-\operatorname{ch} \sigma x) \operatorname{ch} x_{0}+(\operatorname{sh} \sigma s-\operatorname{sh} \sigma x) \operatorname{sh} x_{0}\right)^{\gamma-1} \operatorname{sh} s d s
$$

and $G_{1}(x)$ is defined by replacing $\operatorname{sh} s d s$ with $\operatorname{ch} s d s$. Since $F_{0}$ is supported on $B(r)$, clearly $G_{0}$ is also supported on $B(r)$. Therefore, $W_{-\delta}^{\sigma}(F)$ is supported on $R\left(x_{0}, r\right)$.

Let $a$ be a $(1, \infty, 0,1)$-atom supported on $R\left(x_{0}, r\right)$. By (59) and (58), $\|a\|_{\infty} \leq$ $\left|R\left(x_{0}, r\right)\right|^{-1}(1+r)^{-1}$ and $\int_{G} a(g) d g=0$. We put $A=W_{+}(a)$. Then $A$ is supported on $R\left(x_{0}, r\right)$ by Lemma 5.11 and

$$
\int_{-\infty}^{\infty} A(x) d x=A^{\sim}(0)=\hat{a}(i \rho)=\int_{G} a(g) d g=0 .
$$

Moreover, we recall that $|A(x)| \leq c e^{2 \rho x} \operatorname{th}\left(x_{0}+r\right)^{2 s_{\alpha}}\|a\|_{\infty}$ (see (15), (17) and cf. [10, Lemma 3.3]).

Case I: $x_{0}-r \geq 1$. Since $A$ is supported on $R\left(x_{0}, r\right)$ and

$$
\left|R\left(x_{0}, r\right)\right| \sim \int_{x_{0}-r}^{x_{0}+r} e^{2 \rho x} d x \sim e^{2 \rho x_{0}} \operatorname{sh} r
$$

it follows that $|A(x)| \leq c e^{2 \rho\left(x_{0}+r\right)}\left(e^{2 \rho x_{0}} \operatorname{sh} r\right)^{-1}(1+r)^{-1} \leq c r^{-1}$.

Case II: $x_{0}-r<1$ and $r \geq 1$. Since $x_{0}+r \geq 1$,

$$
\left|R\left(x_{0}, r\right)\right| \geq c \int_{1}^{x_{0}+r} e^{2 \rho x} d x \sim e^{2 \rho\left(x_{0}+r\right)} .
$$

Therefore, as in Case I, we have $\|A\|_{\infty} \leq c r^{-1}$.

Case III: $x_{0}-r<1, r<1$ and $x_{0}>2 r$. Since $x_{0}>2 r$, it follows that $x_{0}+r \geq 3$ and thus

$$
\left|R\left(x_{0}, r\right)\right| \sim \int_{x_{0}-r}^{x_{0}+r} x^{2 s_{\alpha}} d x \leq c\left(x_{0}-r\right)^{s_{\alpha}} r
$$

Then, since $\left(x_{0}+r\right) /\left(x_{0}-r\right) \leq 3,|A(x)| \leq c \operatorname{th}\left(x_{0}+r\right)^{s_{\alpha}}\left(\left(x_{0}-r\right)^{s_{\alpha}} r\right)^{-1} \leq c r^{-1}$.

Case IV: $x_{0}-r<1, r<1$ and $x_{0} \leq 2 r$. Since $x_{0}+r \leq 3 r<3$ and $\left|R\left(x_{0}, r\right)\right| \geq|B(r)| \sim|B(3 r)|$, we may suppose that $a$ is a centered atom supported on $B(3 r)$. Then $|A(x)| \leq c(\text { th } 3 r)^{s_{\alpha}}|B(3 r)|^{-1} \leq c r^{-1}$.

These four cases imply that $c A$ is a $(1, \infty, 0)$-atom on $\mathbf{R}$ and $c$ is independent of $a$. 
TheOREM 5.12. Let $M \geq 2$. Then $M_{\phi}$ is bounded from $\mathrm{H}_{\infty, 0}^{1}(G / / K)$ to $L^{1}(G / / K)$, that is, there exists a constant $c>0$ such that

$$
\left\|M_{\phi} f\right\|_{1} \leq c\|f\|_{\mathrm{H}_{\infty, 0}^{1}(G)}
$$

for all $f \in \mathrm{H}_{\infty, 0}^{1}(G / / K)$ and thus,

$$
\mathrm{H}_{\infty, 0}^{1}(G / / K) \subset H^{1}(G / / K)
$$

Proof. Since $M_{\phi}$ is sublinear and $\left\|M_{\phi} f_{x}^{b}\right\|_{1} \leq\left\|M_{\phi} f\right\|_{1}$, in order to obtain the $\mathrm{H}_{\infty, 0}^{1} L^{1}$ boundedness of $M_{\phi}$, it is enough to show that there exists a constant $c>0$ such that $\left\|M_{\phi} a\right\|_{1} \leq c$ for all centered $(1, \infty, 0)$-atoms $a$ on $G$. Let $B(r)$ denote the support of $a$. Here we recall Theorem 4.5 in [7] and the proof. Since $|B(r)|^{-1} \chi_{B(r)}(x)$ is an $L^{1}$ non-increasing denominator of $a, a$ has a $(1, \infty, 0)$-atomic decomposition $a=\sum_{i} \lambda_{i} a_{i}$ on $G$ such that $\sum_{i}\left|\lambda_{i}\right| \leq c$, where each atom $a_{i}$ has radius $r_{i} \leq 1$. This means that $a_{i}$ is a $(1, \infty, 0,1)$-atom on $G$. Hence, it follows from Theorem 5.10 that $\left\|M_{\phi} a_{i}\right\| \leq c$ and thus, $\left\|M_{\phi} a\right\|_{1} \leq c$, where $c$ is independent of $a$.

\section{Atomic decomposition of $H^{1}(G / / K)$}

We shall prove that each function $f$ in $H^{1}(G / / K)$ has a $(1, \infty,+)$-atomic decomposition on $G$. This means that $H^{1}(G / / K) \subset H_{\infty, 0}^{1,+}(G / / K)$ and then our main Theorem $\mathrm{C}$ in $\S 1$ follows. In the following, first we shall introduce a space $W_{-}\left(H_{\infty, 0}^{1,+}(\mathbf{R})_{\alpha}\right)$ and give a $(1, \infty,+)$-atomic decomposition on $G$ for this space. Then, we shall prove that $H^{1}(G / / K) \subset W_{-}\left(H_{\infty, 0}^{1,+}(\mathbf{R})_{\alpha}\right)$ and obtain the desired $(1, \infty,+)$-atomic decomposition for $H^{1}(G / / K)$.

We set

$$
d_{\alpha}\left(x_{0}, r\right)=\int_{x_{0}-r}^{x_{0}+r}|\operatorname{th} x|^{s_{\alpha}} d x
$$

and define $H_{\infty, 0}^{1,+}(\mathbf{R})_{\alpha}$ as the space of all $F=\sum_{i} \lambda_{i} A_{i}$ such that $\sum_{i}\left|\lambda_{i}\right|<\infty$ and each $A_{i}$ satisfies

$$
\begin{gathered}
\operatorname{supp}\left(A_{i}\right) \subset R\left(x_{i}, r_{i}\right) \\
\left\|W_{-s_{\alpha}}^{\mathbf{R}}\left(A_{i}\right)\right\|_{\infty} \leq d_{\alpha}\left(x_{i}, r_{i}\right)^{-1} \\
\int_{-\infty}^{\infty} A_{i}(x) d x=0 \text { if } r_{i}<1
\end{gathered}
$$

Definition 6.1. We define

$$
W_{-}\left(H_{\infty, 0}^{1,+}(\mathbf{R})_{\alpha}\right)=\left\{f \in L_{\mathrm{loc}}^{1}(G / / K) ; W_{+}(f) \in H_{\infty, 0}^{1,+}(\mathbf{R})_{\alpha}\right\}
$$


Proposition 6.2. Functions in $W_{-}\left(H_{\infty, 0}^{1,+}(\mathbf{R})_{\alpha}\right)$ have $(1, \infty,+)$-atomic decompositions, that is, $W_{-}\left(H_{\infty, 0}^{1,+}(\mathbf{R})_{\alpha}\right) \subset H_{\infty, 0}^{1,+}(G / / K)$.

Proof. Let $f \in W_{-}\left(H_{\infty, 0}^{1,+}(\mathbf{R})_{\alpha}\right)$ and $F=W_{+}(f)=\sum_{i} \lambda_{i} A_{i}$ the decomposition of $F$ given by (66). By using the same argument as in the proof of Proposition 5.5 (see (64)), we may suppose that $r_{i} \leq 1$ in (66). More precisely, when $r_{i}>1$, we decompose the support of $A_{i}$ by using a smooth decomposition of 1 , where each piece is supported in the interval with radius $\leq 1$. Then $A_{i}=\sum_{j} A_{i j}$ and each $A_{i j}$ satisfies (66) with radius $\leq 1$ by Lemma 4.4 (3). Hence, we may suppose $r_{i} \leq 1$ to begin with.

When $\left|x_{i}\right| \leq 2 r_{i}, A_{i}$ is supported on $B\left(r_{i}^{\prime}\right), r_{i}^{\prime}=\left|x_{i}\right|+r_{i} \leq 3 r_{i}$, and satisfies (ii) and (iii) with $r_{i}^{\prime} \leq 3$, because

$$
d_{\alpha}\left(x_{i}, r_{i}\right) \geq d_{\alpha}\left(0, r_{i}\right) \sim r_{i}^{s_{\alpha}+1} \sim d_{\alpha}\left(0,3 r_{i}\right) .
$$

Hence, we may suppose that $x_{i}=0$ with $r_{i} \leq 3$ or $\left|x_{i}\right|>2 r_{i}$. We recall that $e^{-\rho x} W_{+}(f)(x)=F_{f}^{0}(x)$ is an even function on $\mathbf{R}$ (cf. [11, (3.6)]). Therefore, in the decomposition $e^{-\rho x} F=\sum_{i} \lambda_{i} e^{-\rho x} A_{i}$, the counterparts $e^{\rho x} A_{i}(-x)$ must appear in the decomposition. When $x_{i}=0$ with $r_{i} \leq 3$, we may suppose that $e^{-\rho x} A_{i}(x)$ is even. Actually, we let

$$
\left(e^{-\rho x} A_{i}(x)+e^{\rho x} A_{i}(-x)\right)=2\left(1+e^{2 \rho r_{i}}\right) \cdot e^{-\rho x} B_{i}(x) .
$$

Then $B_{i}$ is supported on $B\left(r_{i}\right)$ and $e^{-\rho x} B_{i}(x)$ is even. Moreover, if $r_{i}<1$, then $\int B_{i}(x) d x=0$, because $A_{i}$ satisfies (iii) originally and the even property of $e^{-\rho x} F(x)$ implies that $\int_{-\infty}^{\infty} e^{2 \rho x} A_{i}(-x) d x=0$. Since $r_{i} \leq 3, B_{i}$ also satisfies (ii) and $2\left(1+e^{2 \rho r_{i}}\right) \sim 1$. Therefore, replacing the left hand side with the right one, we may suppose that $e^{-\rho x} A_{i}(x)$ is even if $x_{i}=0$ with $r_{i} \leq 3$. Thereby, we can rearrange the decomposition of $F$ as

$$
F=\sum_{i} \lambda_{i} A_{i}+\sum_{j} \mu_{j} B_{j}+\sum_{k} \gamma_{k} E_{k}
$$

where each $A_{i}$ satisfies $(i),(i i)$ with $x_{i}=0, r_{i} \leq 3, \int A_{i}(x) d x=0$; each $B_{j}$ satisfies (i) to (iii) with $\left|x_{j}\right| \geq 2 r_{j}, r_{j}<1$; each $E_{k}$ satisfies (i), (ii) with $\left|x_{k}\right| \geq 2 r_{k}, r_{k} \geq 1$, and moreover, $\sum_{i}\left|\lambda_{i}\right|+\sum_{j}\left|\mu_{j}\right|+\sum_{k}\left|\gamma_{k}\right|<\infty$. Since $F$ is $W_{+}$-smooth, finally, we have

$$
f=\sum_{i} \lambda_{i} a_{i}+\sum_{j} \mu_{j} b_{j}+\sum_{k} \gamma e_{k}
$$

where $a_{i}=W_{-}\left(A_{i}\right), b_{j}=W_{-}\left(B_{j}\right)$ and $e_{k}=W_{-}\left(E_{k}\right)$. Lemma 5.11 implies that each $a_{i}, b_{j}, e_{k}$ have the same supports as $A_{i}, B_{j}, E_{k}$ respectively. 
Now we apply fractional calculus in [10] to estimate each $a_{i}, b_{j}, e_{k}$. For simplicity, we skip writing the suffices $i, j, k$ and denote the supports of $a, b, e$ by $R\left(x_{0}, r\right)$. Without loss of generality, we may suppose that $x_{0} \geq 0$.

As for $e$, since $e$ is supported on $R\left(x_{0}, 1\right)$ and $x_{0} \geq 2$, it follows that $x_{0}-1 \geq 1$ and thus, $d_{\alpha}\left(x_{0}, 1\right) \sim 1$. Thereby, $(i i)$ and [10, Lemma 3.3 $]^{1}$ imply that on the support of $e$

$$
|e(x)| \leq c(\operatorname{th} x)^{-(\alpha+1 / 2)} e^{-2 \rho x} \leq c e^{-2 \rho x} \leq c|R(x, 1)|^{-1} .
$$

This means that $c^{-1} e$ is a $(1, \infty,+)$-atom on $G$.

As for $b$, we recall that $x_{0}=0$ or $x_{0}>2 r$.

Case I. $\quad x_{0}-r \geq 1$ : Since $x_{0}-r \geq 1, d_{\alpha}\left(x_{0}, r\right) \sim r$. Thereby, $(i i)$ and [10, Lemma 3.3] imply that on the support of $b$

$$
|b(x)| \leq c(\operatorname{th} x)^{-(\alpha+1 / 2)} e^{-2 \rho x} r^{-1} \leq c e^{-2 \rho x} r^{-1} \leq c|R(x, r)|^{-1} .
$$

This means that $c^{-1} b$ is a $(1, \infty, 0)$-atom on $G$.

Case II. $x_{0}-r<1$ : Since $r<1$ and $x_{0}>2 r$, it follows that $x_{0}<r+1<2$, $x_{0}-r>x_{0} / 2$, and $x_{0}+r<3 x_{0} / 2<3$. Therefore, $d_{\alpha}\left(x_{0}, r\right) \leq c\left(x_{0}-r\right)^{s_{\alpha}} r$ and thus, on the support of $b$

$$
|b(x)| \leq c(\operatorname{th} x)^{-(\alpha+1 / 2)} e^{-2 \rho x} r^{-1}\left(x_{0}-r\right)^{-s_{\alpha}} \leq c\left(x_{0}-r\right)^{-(2 \alpha+1)} r^{-1} .
$$

Since $\left(x_{0}+r\right) /\left(x_{0}-r\right) \leq 3$, it follows that

$$
\left|R\left(x_{0}, r\right)\right| \leq c\left(x_{0}+r\right)^{2 \alpha+1} r \leq c\left(x_{0}-r\right)^{2 \alpha+1} r .
$$

Therefore, $|b(x)| \leq c\left|R\left(x_{0}, r\right)\right|^{-1}$ on the support. This means that $c^{-1} b$ is a $(1, \infty, 0)$-atom on $G$.

As for $a$, since $x_{0}=0$ and $r<1$, it follows that $d_{\alpha}(0, r) \sim r^{s_{\alpha}+1}$ and

$$
|a(x)| \leq c(\operatorname{th} x)^{-(\alpha+1 / 2)} e^{-2 \rho x} r^{-1} r^{-\left(s_{\alpha}+1\right)} \leq c \Delta(x)^{-1} r^{-1} .
$$

We put

$$
a_{+}(x)=c \Delta(x)^{-1} r^{-1} \chi_{[0, r]}(x), \quad x>0 .
$$

Clearly, $|a(x)| \leq a_{+}(x)$ and $a_{+}$is a non-increasing function on $\mathbf{R}_{+}$with finite $L^{1}$-norm:

$$
\left\|a_{+}\right\|_{L^{1}(\Delta)}=\int_{0}^{\infty} a_{+}(x) \Delta(x) d x \leq c_{0} .
$$

\footnotetext{
${ }^{1}$ If $f(x)=O\left(x^{2 \tau}\right)$ around $x=0$, then $W_{-\mu}^{\sigma}(f)(x)=O\left(x^{2(\tau-\Re \mu)}\right)$. However, under the assumption (ii) of (66), it follows that $W_{-\mu}^{\sigma}(f)(x)=O\left(x^{-\Re \mu}\right)$.
} 
Since $a$ is supported on $B(r)$ and $\int_{G} a(g) d g=\int_{-\infty}^{\infty} A(x) d x=0$, it follows that $|B(s)|^{-1} \int_{s}^{\infty} a(x) \Delta(x) d x$ is also supported on $B(r)$ and

$$
\frac{1}{|B(s)|} \int_{s}^{\infty} a(x) \Delta(x) d x=\frac{1}{|B(s)|} \int_{0}^{s} a(x) \Delta(x) d x \leq c \Delta(s)^{-1} r^{-1}
$$

Here we used (68) and $|B(s)| \sim \Delta(s) s$ if $s \leq r \leq 1$ (see (4)). Hence,

$$
\frac{1}{|B(s)|} \int_{s}^{\infty} a(x) \Delta(x) d x \leq a_{+}(s) \text {. }
$$

This means that $c a_{+}$is an $L^{1}$ non-increasing denominator of $a$ satisfying (70). Then [7, Theorem 4.5] yields that $a$ has a centered $(1, \infty, 0)$-atomic decomposition $a=$ $\sum_{j} \gamma_{j} a_{j}$ on $G$ such that $\sum_{j}\left|\gamma_{j}\right| \leq c\left\|a_{+}\right\|_{L^{1}(\Delta)} \leq c c_{0}$. Especially, $a \in H_{\infty, 0}^{1}(G / / K)$ and $\|a\|_{H_{\infty, 0}^{1}(G)} \leq c c_{0}$.

These three cases imply that all $a_{i}, b_{j}, e_{k}$ in (67), and thus $f$ also, belong to $H_{\infty, 0}^{1,+}(G / / K)$. This completes the proof of the proposition.

Theorem 6.3. Let notations be as above. Then

$$
\begin{aligned}
H^{1}(G / / K) & =H_{\infty, 0}^{1,+}(G / / K)^{\sharp} \cap W_{-}\left(H^{1}(\mathbf{R})\right) \\
& =H_{\infty, 0}^{1,+}(G / / K) \cap W_{-}\left(H^{1}(\mathbf{R})\right) .
\end{aligned}
$$

Proof. Because of Proposition 5.9 and Corollary 4.3, it is enough to prove that $H^{1}(G / / K) \subset H_{\infty, 0}^{1,+}(G / / K)$. First we shall consider the case that $\delta=\delta^{\prime}=0$, that is, $s_{\alpha}$ is an integer (see (37)).

Let $f \in H^{1}(G / / K)$ and put $F=W_{+}(f)$. Then it follows from Theorem 4.6 that $\left\|M_{\phi}^{\mathbf{R}} \circ W_{-s_{\alpha}}^{\mathbf{R}}(F)(x)(\operatorname{th} x)^{s_{\alpha}}\right\|_{L^{1}(\mathbf{R})}<\infty$. We here recall the constructive proof of the atomic decomposition of $H^{1}(\mathbf{R})$ : For example, we refer to [12]. Then, measuring the volume of the dyadic cubes (intervals in the one-dimensional case) $Q_{j}^{k}$ appeared in the Whitney decomposition theorem by (65) and keeping the convolution and the dilation, we can easily deduce that $W_{-s_{\alpha}}^{\mathbf{R}}(F)$ has a $\left(1, \infty, s_{\alpha}\right)$-atomic decomposition with respect to $\mid$ th $\left.x\right|^{s_{\alpha}} d x$ :

$$
W_{-s_{\alpha}}^{\mathbf{R}}(F)=\sum_{i} \lambda_{i} B_{i}
$$

where $B_{i}$ is supported on $R\left(x_{i}, r_{i}\right), \int_{-\infty}^{\infty} B_{i}(x) x^{k} d x=0,0 \leq k \leq s_{\alpha},\left\|B_{i}\right\|_{\infty} \leq$ $d_{\alpha}\left(x_{i}, r_{i}\right)^{-1}$, and $\sum_{i}\left|\lambda_{i}\right|<\infty$. We set

$$
F=\sum_{i} \lambda_{i} W_{s_{\alpha}}^{\mathbf{R}}\left(B_{i}\right)=\sum_{i} \lambda_{i} A_{i}
$$

Since $s_{\alpha}$ is an integer and each $B_{i}$ satisfies the $s_{\alpha}$-th moment condition, it follows that $A_{i}$ is supported on $R\left(x_{i}, r_{i}\right)$ and $\int_{-\infty}^{\infty} A_{i}(x) d x=0$. Moreover, $\left\|W_{-s_{\alpha}}^{\mathbf{R}}\left(A_{i}\right)\right\|_{\infty}=$ 
$\left\|B_{i}\right\|_{\infty} \leq d_{\alpha}\left(x_{i}, r_{i}\right)^{-1}$. Therefore, $A_{i}$ satisfies (66) and thus, $F \in H_{\infty, 0}^{1,+}(\mathbf{R})_{\alpha}$. Hence Proposition 6.2 implies that $f \in H_{\infty, 0}^{1,+}(G / / K)$.

We shall remove the assumption that $\delta=\delta^{\prime}=0$ and consider a general case of $0 \leq \delta, \delta^{\prime}<1$. We recall the Fourier-Jacobi analysis (cf. [4], [11]) and note that all results obtained in the previous sections can be extended to the Fourier-Jacobi analysis, that is, for arbitrary $\alpha, \beta \geq-1 / 2$. Especially, we may denote $W_{ \pm}=W_{ \pm}^{\alpha, \beta}$ and replace " $G / / K$ " as " $(\alpha, \beta)$ " such as

$$
H^{1}(G / / K)=H^{1}(\alpha, \beta)
$$

For $f \in H^{1}(\alpha, \beta)$, we set

$$
\begin{aligned}
F=W_{+}^{\alpha, \beta}(f) & =e^{\rho x} W_{\alpha-\beta}^{1} \circ W_{\beta+1 / 2}^{2}(f) \\
& =e^{\rho x} W_{\delta}^{1} \circ W_{n}^{1} \circ W_{n^{\prime}}^{2} \circ W_{\delta^{\prime}}^{2}(f) \\
& =W_{+}^{n, n^{\prime}}\left(S_{\delta, \delta^{\prime}}(f)\right)
\end{aligned}
$$

where $\rho=\alpha+\beta+1$ and

$$
S_{\delta, \delta^{\prime}}(f)=W_{-}^{n, n^{\prime}} \circ W_{+}^{\alpha, \beta}(f) .
$$

We note that Theorem 4.6 means that

$$
\sum_{m=0}^{\underline{n}}\left\|M_{\phi}^{\mathbf{R}} \circ W_{-m}^{\mathbf{R}}(F)\right\|_{L^{1}(\mathbf{R})}<\infty
$$

and thus, $S_{\delta, \delta^{\prime}}(f)$ belongs to $H^{1}\left(n, n^{\prime}\right)$. Then, since $n, n^{\prime}$ are integers (see (36)), $S_{\delta, \delta^{\prime}}(f)$ belongs to $H_{\infty, 0}^{1,+}\left(n, n^{\prime}\right)$ and it has a $(1, \infty,+)$-atomic decomposition with respect to $\left(n, n^{\prime}\right)$ :

$$
S_{\delta, \delta^{\prime}}(f)=\sum_{i} \lambda_{i} a_{i}
$$

where $\sum_{i}\left|\lambda_{i}\right|<\infty$. Here

$$
F=\sum_{i} \lambda_{i} A_{i}, \quad A_{i}=W_{+}^{n, n^{\prime}}\left(a_{i}\right)
$$

and each $A_{i}$ satisfies (66) with respect to $\left(n, n^{\prime}\right)$. We here recall that $F$ is originally $W_{+}$-smooth. Hence, from a constructive proof of atomic decompositions $W_{-\left(n+n^{\prime}\right)}^{\mathbf{R}}(F)=\sum_{i} \lambda_{i} B_{i}$ (cf. [6, B in Chap. 3]) it follows that $A_{i}$ satisfies (66) with respect to $(\alpha, \beta)$. We let $b_{i}=W_{-}\left(W_{+}^{n, n^{\prime}}\left(a_{i}\right)\right)$. Each $b_{i}$ has the same support as $a_{i}$ by Lemma 5.11 and $\int_{G} b_{i}(g) d g=A_{i}^{\sim}(0)=0$ if $r_{i}<1$. Moreover, we see from [10, Lemma 3.3] (or the same arguments in the case of $\delta=\delta^{\prime}=0$ ) that 
$\left\|b_{i}\right\|_{\infty} \leq c\left|R\left(x_{i}, r_{i}\right)\right|^{-1}$. Thereby, we see that $f=\sum_{i} \lambda_{i} b_{i}$ is a $(1, \infty,+)$-atomic decomposition of $f$.

Theorem 6.4. Let $\epsilon \geq 0$. Then $H_{\infty, 0}^{1, \epsilon}(G / / K) \cap W_{-}\left(H^{1}(\mathbf{R})\right)$ is dense in $W_{-}\left(H^{1}(\mathbf{R})\right)$. Especially, $H^{1}(G / / K)$ is dense in $W_{-}\left(H^{1}(\mathbf{R})\right)$.

Proof. Let $f \in W_{-}\left(H^{1}(\mathbf{R})\right)$. We approximate $f$ with a rapidly decreasing function in $W_{-}\left(H^{1}(\mathbf{R})\right)$. For example, let us take $f_{1}=f * \phi_{t}$ (see $\S 3$ ). Since $W_{+}\left(f_{1}\right)$ $=W_{+}(f) * W_{+}\left(\phi_{t}\right)$ and $W_{+}\left(\phi_{t}\right)$ satisfies Lemma 3.6, it is easy to see that $F_{1}=$ $W_{+}\left(f_{1}\right) \in H^{1}(\mathbf{R})$ and $\left\|f-f_{1}\right\|_{H^{1}(G)}=\left\|F-F_{1}\right\|_{H^{1}(\mathbf{R})}=\left\|F-F * W_{+}\left(\phi_{t}\right)\right\|_{H^{1}(\mathbf{R})} \rightarrow 0$ as $t \rightarrow 0$ (cf. [15; Chap. 3, 5.1 (d)]). We fix a sufficiently small $t>0$ and let $F_{1}=W_{+}\left(f * \phi_{t}\right)=\sum_{i \in I} \lambda_{i} A_{i}$ denote a $(1, \infty, 0)$-atomic decomposition of $F_{1}$. For a sufficiently small $\kappa>0$ and a finite large index set $J \subset I$, we put

$$
F_{0}=\sum_{i \in J_{0}} \lambda_{i} A_{i}, \quad J_{0}=\left\{j \in J ; r_{i} \geq \kappa\right\}
$$

where we take $\kappa$ and $J$ for which $e^{-\rho x} F_{0}(x)$ is even. We here recall a constructive proof of the atomic decomposition of $H^{1}(\mathbf{R})$ (cf. [12] and [6, B in Chap. 3]). Since $F_{1}$ is smooth, each $A_{i}$ is also smooth and if $i \in J_{0}, r_{i} \leq 1$, then

$$
\left\|W_{-s_{\alpha}}^{\mathbf{R}}\left(A_{i}\right)\right\|_{\infty} \leq c r_{i}^{-\left(s_{\alpha}+1\right)} \leq c \kappa^{-s_{\alpha}} r_{i}^{-1}
$$

and if $i \in J_{0}, r_{i} \geq 1$, then

$$
\left\|W_{-s_{\alpha}}^{\mathbf{R}} A_{i}\right\|_{\infty} \leq c r_{i}^{-1} .
$$

In particular, $\left\|W_{-s_{\alpha}}^{\mathbf{R}} A_{i}\right\|_{\infty} \leq R\left(x_{i}, r_{i}\right)^{-1}$ if $\operatorname{supp}\left(A_{i}\right)=R\left(x_{i}, r_{i}\right)$. Hence, each $A_{i}$, $i \in J_{0}, r_{i} \leq 1$, satisfies (66) and thus, $F_{0} \in H_{\infty, 0}^{1,+}(\mathbf{R})_{\alpha}$. Then, by Proposition 6.2 , we can define $f_{0} \in H_{\infty, 0}^{1,+}(G / / K)$ such that $W_{+}\left(f_{0}\right)=F_{0}$. Since $F_{0} \in H^{1}(\mathbf{R})$, it follows that $f_{0} \in H_{\infty, 0}^{1,+}(G / / K) \cap W_{-}\left(H^{1}(\mathbf{R})\right)$ and $\left\|f_{1}-f_{0}\right\|_{H^{1}(G)}=\left\|F_{1}-F_{0}\right\|_{H^{1}(\mathbf{R})} \rightarrow 0$ if $\kappa \rightarrow 0$ and $J \rightarrow I$. This means that $H_{\infty, 0}^{1,+}(G / / K) \cap W_{-}\left(H^{1}(\mathbf{R})\right)$ is dense in $W_{-}\left(H^{1}(\mathbf{R})\right)$. Since

$$
H_{\infty, 0}^{1,+}(G / / K) \cap W_{-}\left(H^{1}(\mathbf{R})\right) \subset H^{1}(G / / K) \subset W_{-}\left(H^{1}(\mathbf{R})\right)
$$

(see Proposition 5.9 and Corollary 4.3), in order to obtain the desired density it is enough to show that $f_{0}$ belongs to $H_{\infty, 0}^{1, \epsilon}(G / / K)$.

We keep the atomic decomposition of $F_{0}$ in (71). Let $I_{i}=R\left(x_{i}, r_{i}\right)$ denote the support of $A_{i}$. As in the proof of Proposition 6.2, we may suppose that $x_{i}=0$ or $x_{i}>2 r_{i}$. We recall that $e^{-\rho x} F_{0}$ is even and $I_{i} \cap-I_{i}=\emptyset$ if $0 \notin I_{i}$. When $x_{i}=0$, we put

$$
\left(e^{-\rho x} A_{i}(x)+e^{\rho x} A_{i}(-x)\right)=2\left(1+e^{2 \rho r_{i}}\right) \cdot e^{-\rho x} B_{i}(x) .
$$


Then $B_{i}$ is a $(1, \infty, 0)$-atom on $\mathbf{R}$ and $e^{-\rho x} B_{i}(x)$ is even. Since the index set $J_{0}$ is finite, changing the coefficient $\lambda_{i}$ by $2 \lambda_{i} e^{2 \rho r_{i}}$ and replacing the left hand side with. the right one, we may suppose that $e^{-\rho x} A_{i}(x)$ is even if $x_{i}=0$. Since each $A_{i}$ is piecewise $W_{+}$-smooth, we can define a $K$-bi-invariant function $a_{i}=W_{-}\left(A_{i}\right)$ on $G$ such that

$$
f_{0}=\sum_{i \in J_{0}} \lambda_{i} a_{i}
$$

Case I. $x_{i}>2 r_{i}$ and $r_{i}<1$ : As in the proof of Proposition 6.2, each $a_{i}$ is a $(1, \infty, 0)$-atom on $G$ up to a constant multiplication.

Case II. $x_{i}>2 r_{i}$ and $r_{i} \geq 1$ : Since $x_{i}>2 r_{i}$, it follows that $x_{i}+r_{i} \geq 3$ and $x_{i}-r_{i} \geq 1$. Thereby, $\left|R\left(x_{i}, r_{i}\right)\right| \sim e^{2 \rho\left(x_{i}+r_{i}\right)}$. Since $J_{0}$ is finite, we may replace $\lambda_{i} a_{i}$ as

$$
\lambda_{i} a_{i}=\lambda_{i} e^{4 \rho r_{i}} r_{i}^{\epsilon} \cdot b_{i}, \quad b_{i}=e^{-4 \rho r_{i}} r_{i}^{-\epsilon} a_{i} .
$$

Then, similarly as for (68), we can deduce from (72) that

$$
\left\|b_{i}\right\|_{\infty} \leq c e^{-4 \rho r_{i}} r_{i}^{-\epsilon} \Delta\left(x_{i}-r_{i}\right)^{-1} r_{i}^{-1} \leq c\left|R\left(x_{i}, r_{i}\right)\right|^{-1} r_{i}^{-\epsilon} .
$$

This means that $a$ is a $(1, \infty, 0, \epsilon)$-atom on $G$ up to a constant multiplication.

Case III. $x_{i}=0$ and $r_{i} \leq 1$ : As in the proof of Proposition 6.2, each $a_{i}$ has a $(1, \infty, 0)$-atomic decomposition.

Case IV. $\quad x_{i}=0$ and $r_{i}>1$ : Since $J_{0}$ is finite, we may replace $\lambda_{i} a_{i}$ as

$$
\lambda_{i} a_{i}=\lambda_{i} r_{i} \cdot b_{i}, \quad b_{i}=r_{i}^{-1} a_{i} .
$$

Then, similarly as for (68), we can deduce from (72) that

$$
\left|b_{i}(x)\right| \leq c(\operatorname{th} x)^{-(2 \alpha+1)} e^{-2 \rho x} r_{i}^{-2} \leq c \Delta(x) r_{i}^{-2} .
$$

We set $b_{+}(x)=\Delta(x)^{-1} r_{i}^{-1}$. As in (69), using the moment condition, we see that

$$
\frac{1}{|B(s)|} \int_{s}^{\infty} b_{i}(x) \Delta(x) d x \leq \frac{s}{|B(s)|} r_{i}^{-2} \leq b_{+}(s), \quad 0<s \leq r_{i} .
$$

Therefore, $b_{+}$is an $L^{1}$ non-increasing denominator of $b_{i}$ satisfying (74). Hence, $b_{i}$ has a $(1, \infty, 0)$-atomic decomposition (see [7, Theorem 4.5$]$ ).

These four cases yield that $f_{0} \in H_{\infty, 0}^{1, \epsilon}(G / / K)$.

REMARK 6.5. As in the proof of Theorem 6.4, we set

$$
S_{\gamma, \gamma^{\prime}}(f)=W_{-}^{\alpha-\gamma, \beta-\gamma^{\prime}} \circ W_{+}^{\alpha, \beta}(f)
$$


for $0 \leq \gamma \leq \alpha+1 / 2,0 \leq \gamma^{\prime} \leq \beta+1 / 2$. Clearly, $S_{0,0}$ is the identity operator and $S_{\alpha+1 / 2, \beta+1 / 2}=W_{+}^{\alpha, \beta}$. Then it follows that

$$
H^{1}(\alpha, \beta) \subset S_{\gamma, \gamma^{\prime}}^{-1}\left(H^{1}\left(\alpha-\gamma, \beta-\gamma^{\prime}\right)\right)
$$

When $\gamma=\alpha+1 / 2$ and $\gamma^{\prime}=\beta+1 / 2$, this relation is nothing but the one in Corollary 4.3 , because $H^{1}(-1 / 2,-1 / 2)=H^{1}(\mathbf{R})$.

\section{Other operators}

We shall consider $\left(H^{1}, L^{1}\right)$-boundedness of singular integrals, heat and Poisson maximal operators $M_{\mathbf{H}}$ and $M_{\mathbf{P}}$, and the Riesz transform $\mathrm{R}$ on $G$.

Let $T_{m}^{\mathbf{R}}=\mathrm{M}_{m}$ be a Fourier multiplier corresponding to a bounded function $m$ and $K$ the distribution kernel of $\mathrm{M}_{m}$, that is, $K^{\sim}(\lambda)=m(\lambda)$ and $\mathrm{M}_{m}(F)=K * F$ in a distribution sense (cf. $[15$, Chapter $1, \S 6]$ ). We put $k=W_{-}(K)$ and define an operator $T_{m}^{G}$ on $G$ as $T_{m}^{G}(f)=k * f$. Then it is easy to see from (22), Definition 4.2, Theorem 4.6 that $\left\|T_{m}^{G}(f)\right\|_{W_{-}\left(H^{1}(\mathbf{R})\right)}=\left\|W_{+}(k * f)\right\|_{H^{1}(\mathbf{R})}=\left\|T_{m}^{\mathbf{R}}\left(W_{+}(f)\right)\right\|_{H^{1}(\mathbf{R})}$ and $\left\|T_{m}^{G}(f)\right\|_{H^{1}(G)} \leq c\left\|\mathrm{M}_{s_{\alpha}} W_{+}\left(T_{m}^{G}(f)\right)\right\|_{H^{1}(\mathbf{R})}=\left\|\mathrm{M}_{s_{\alpha}} \circ T_{m}^{\mathbf{R}}\left(W_{+}(f)\right)\right\|_{H^{1}(\mathbf{R})}$. Therefore, we have the following.

THEOREM 7.1. Let notation be as above. If $T_{m}^{\mathbf{R}}$ is bounded on $H^{1}(\mathbf{R})$, then $T_{m}^{G}$ is bounded on $W_{-}\left(H^{1}(\mathbf{R})\right)$. Moreover, if $\mathrm{M}_{s_{\alpha}} \circ T_{m}^{\mathbf{R}}$ is bounded on $H^{1}(\mathbf{R})$, then $T_{m}^{G}$ is bounded from $W_{-}\left(H^{1}(\mathbf{R})\right)$ to $H^{1}(G / / K)$, especially, it is bounded on $H^{1}(G / / K)$.

Let $h_{t}(g)$ and $p_{t}(g), g \in G, t>0$, denote the heat and Poisson kernels on $G$. They are $K$-bi-invariant functions on $G$ whose spherical Fourier transforms are respectively given by

$$
\hat{h}_{t}(\lambda)=e^{-t\left(\lambda^{2}+\rho^{2}\right)} \quad \text { and } \quad \hat{p}_{t}(\lambda)=e^{-t \sqrt{\lambda^{2}+\rho^{2}}} .
$$

Definition 7.2. For $\epsilon \geq 0$ we define the modified heat and Poisson maximal operators $M_{\mathbf{H}}^{\epsilon}$ and $M_{\mathbf{P}}^{\epsilon}$ on $G$ as follows.

$$
\begin{aligned}
& \left(M_{\mathbf{H}}^{\epsilon} f\right)(g)=\sup _{0<t<\infty}(1+t)^{-\epsilon}\left|\left(f * h_{t}\right)(g)\right|, \\
& \left(M_{\mathbf{P}}^{\epsilon} f\right)(g)=\sup _{0<t<\infty}(1+t)^{-\epsilon}\left|\left(f * p_{t}\right)(g)\right| .
\end{aligned}
$$

For simplicity, we denote $M_{\mathbf{H}}^{0}$ and $M_{\mathbf{P}}^{0}$ by $M_{\mathbf{H}}$ and $M_{\mathbf{P}}$ respectively.

It is well-known that $M_{\mathbf{H}}$ and $M_{\mathbf{P}}$ satisfy the maximal theorem (see [14, p. 73 and p. 48], [1, $\S 3$ and $\S 6]$ ). Moreover, if we define their truncated maximal operators $M_{\mathbf{H}}^{\text {loc }}$ and $M_{\mathbf{P}}^{\text {loc }}$ by restricting the range of the supremum as $0<t<1$ 
(cf. Definition 4.8), then they are bounded from $\mathrm{H}_{\infty, 0}^{1,+}(G / / K)^{\sharp}$ to $L^{1}(G / / K)$ (see [9, Theorem 6.1, Remark 6.5]).

We here introduce a modified atomic Hardy space $H_{\infty, N}^{1, \epsilon}(\mathbf{R}), \epsilon \geq 0, N=$ $0,1,2, \cdots$, on $\mathbf{R}$ by replacing the norm condition $\|A\|_{\infty} \leq r^{-1}$ of a $(1, \infty, N)$-atom $A$ on $\mathbf{R}$ with

$$
\|A\|_{\infty} \leq r^{-1}(1+r)^{-\epsilon}
$$

(cf. Definition 5.1 on $G$ ).

Definition 7.3. We define

$$
W_{-}\left(L^{1}(\mathbf{R})\right)=\left\{f \in L_{\mathrm{loc}}^{1}(G / / K) ; W_{+}(f) \in L^{1}(\mathbf{R})\right\}
$$

and

$$
W_{-}\left(H_{\infty, N}^{1, \epsilon}(\mathbf{R})\right)=\left\{f \in L_{\mathrm{loc}}^{1}(G / / K) ; W_{+}(f) \in H_{\infty, N}^{1, \epsilon}(\mathbf{R})\right\}
$$

We give their norms by $\left\|W_{+}(f)\right\|_{L^{1}(\mathbf{R})}$ and $\left\|W_{+}(f)\right\|_{H^{1}(\mathbf{R})}$ respectively.

Clearly,

$$
\mathrm{H}_{\infty, 0}^{1,+}(G / / K)^{\natural} \subset L^{1}(G / / K) \subset W_{-}\left(L^{1}(\mathbf{R})\right)
$$

(see (20) and Definition 5.6). Since $H_{\infty, N}^{1, \epsilon}(\mathbf{R}) \subset H_{\infty, N}^{1}(\mathbf{R})=H^{1}(\mathbf{R})$ (cf. [6, (3.30)]), it follows that $W_{-}\left(H_{\infty, N}^{1, \epsilon}(\mathbf{R})\right) \subset W_{-}\left(H^{1}(\mathbf{R})\right) \subset W_{-}\left(L^{1}(\mathbf{R})\right)$ and hence, Proposition 5.7 and Theorem 6.3 imply that

$$
\begin{aligned}
H^{1}(G / / K) \cap W_{-}\left(H_{\infty, N}^{1, \epsilon}(\mathbf{R})\right) & =H_{\infty, 0}^{1,+}(G / / K) \cap W_{-}\left(H_{\infty, N}^{1, \epsilon}(\mathbf{R})\right) \\
& =H_{\infty, 0}^{1,+}(G / / K)^{\sharp} \cap W_{-}\left(H_{\infty, N}^{1, \epsilon}(\mathbf{R})\right) .
\end{aligned}
$$

Theorem 7.4. Let notations be as above. Then $M_{\mathbf{H}}^{\epsilon}$ is bounded from

$$
\begin{cases}\mathrm{H}_{\infty, 0}^{1,+}(G / / K)^{\sharp} & \text { if } \epsilon>\frac{1}{2} \\ H^{1}(G / / K) \cap W_{-}\left(H_{\infty, 0}^{1,1-\epsilon}(\mathbf{R})\right) & \text { if } 0<\epsilon \leq \frac{1}{2} \\ H^{1}(G / / K) \cap W_{-}\left(H_{\infty, 1}^{1,3 / 2}(\mathbf{R})\right) & \text { if } \epsilon=0\end{cases}
$$

to $L^{1}(G / / K)$.

Proof. Since $M_{\mathbf{H}}^{\text {loc }}$ is bounded from $\mathrm{H}_{\infty, 0}^{1,+}(G / / K)^{\sharp}$ to $L^{1}(G / / K)$, we may suppose that $t>1$ in the definition of $M_{\mathbf{H}}^{\epsilon}$. Let $M_{\mathbf{H}, \gamma}^{\epsilon, \mathbf{R}}, \gamma \in \mathbf{R}$, denote the maximal operator on $\mathbf{R}$ associated to the Fourier multiplier corresponding to $t^{-\epsilon} \hat{h}_{t}(\lambda+$ $i \rho)(i \lambda)^{\gamma}$ :

$$
M_{\mathbf{H}, \gamma}^{\epsilon, \mathbf{R}}(F)=\sup _{t>1} \frac{1}{t^{\epsilon}} \int_{-\infty}^{\infty} \hat{h}_{t}(\lambda+i \rho)(i \lambda)^{\gamma} F^{\sim}(\lambda) e^{i \lambda x} d \lambda
$$


For $f \in L^{1}(G / / K)$ we put $F=W_{+}(f)$. Then the same argument as that used in the proof of Theorem 4.6 and (54) yield that

$$
\begin{aligned}
\left\|M_{\mathbf{H}}^{\epsilon} f\right\|_{L^{1}(G)} & \leq c \sum_{m=0}^{\underline{n}} \sum_{\xi \in \underline{D}}\left\|M_{\mathbf{H}, 0}^{\epsilon, \mathbf{R}} \circ W_{-(m+\xi)}^{\mathbf{R}}(F)\right\|_{L^{1}(\mathbf{R})} \\
& =c \sum_{m=0}^{\underline{n}} \sum_{\xi \in \underline{D}}\left\|M_{\mathbf{H}, m+\xi}^{\epsilon, \mathbf{R}}(F)\right\|_{L^{1}(\mathbf{R})},
\end{aligned}
$$

if the both sides exist. Let $H_{t}$ denote the heat kernel on $\mathbf{R}$ :

$$
H_{t}(x)=(2 t)^{-1 / 2} e^{-x^{2} / 4 t} .
$$

Since $H_{t}^{\sim}(\lambda)=e^{-t \lambda^{2}}$, it follows that

$$
\hat{h}_{t}(\lambda+i \rho)=c K_{t}^{\sim}(\lambda), \quad K_{t}(x)=H_{t}(x-2 \rho t) .
$$

Hence, (77) can be rewritten as

$$
\left\|M_{\mathbf{H}}^{\epsilon} f\right\|_{L^{1}(G)} \leq c \sum_{m=0}^{\underline{n}} \sum_{\xi \in \underline{D}}\left\|\sup _{t>1} t^{-\epsilon}\left|W_{-(m+\xi)}^{\mathbf{R}}\left(K_{t}\right) * F\right|\right\|_{L^{1}(\mathbf{R})} .
$$

Lemma 7.5. Let $t \geq 1$ and $x, \mu \geq 0$. Then for each $\gamma \geq 0$, there exists $a$ constant $c$ such that

$$
\left|W_{-\mu}^{\mathbf{R}}\left(H_{t}\right)(x)\right| \leq \frac{c}{\sqrt{t}}\left(1+\frac{x}{\sqrt{t}}\right)^{-\gamma} t^{-\mu / 2}
$$

and

$$
\left|W_{-\mu}^{\mathbf{R}}\left(K_{t}\right)(x)\right| \leq \frac{c}{\sqrt{t}}\left(1+\left|\frac{x}{t}-2 \rho\right|\right)^{-\gamma} t^{-\mu / 2} .
$$

Proof. Let $0 \leq \delta<1$ and $x \geq 0$. We note that

$$
\begin{aligned}
W_{-\mu}^{\mathbf{R}}\left(H_{t}\right)(x) & =\frac{c}{\sqrt{t}} \int_{x}^{\infty} e^{-s^{2} / 4 t} \frac{s}{t}(s-x)^{-\mu} d s \\
& \leq \frac{c}{\sqrt{t}} e^{-x^{2} / 4 t} \int_{0}^{\infty} e^{-s^{2} / 4 t} \frac{s+x}{t} s^{-\mu} d s \\
& \leq \frac{c}{\sqrt{t}} e^{-x^{2} / 4 t}\left(1+\frac{x}{\sqrt{t}}\right) t^{-\mu / 2}
\end{aligned}
$$

Then the desired result for $H_{t}$ follows. Since $t \geq 1$, by replacing $x / \sqrt{t}$ by $x / t$ in (81), the one for $K_{t}$ follows from (79). 
We return to the proof of the theorem.

Case I: $\epsilon>1 / 2$. Since $t \geq 1$, Lemma 7.5 with $\gamma=1 / 2+\epsilon$ means that, for $0 \leq m \leq \underline{n}$, and $\xi \in \underline{D},\left|t^{-\epsilon} W_{-(m+\xi)}^{\mathbf{R}}\left(K_{t}\right)(x)\right| \leq c t^{-(\epsilon+1 / 2)}(1+|| x|/ t-2 \rho|)^{-(1 / 2+\epsilon)}$. When $|x| / t>4 \rho, t^{-(\epsilon+1 / 2)}(1+|| x|/ t-2 \rho|)^{-(\epsilon+1 / 2)} \leq c t^{-(\epsilon+1 / 2)}(1+|x| / t \mid)^{-(\epsilon+1 / 2)} \leq$ $c(1+|x|)^{-(\epsilon+1 / 2)}$, because $t \geq 1$, and when $|x| / t \leq 4 \rho, t^{-(\epsilon+1 / 2)}(1+|| x \mid / t-$ $2 \rho \mid)^{-(\epsilon+1 / 2)} \leq c t^{-(\epsilon+1 / 2)} \leq c(1+|x|)^{-(\epsilon+1 / 2)}$. Hence, $t^{-\epsilon}\left|W_{-\left(m+\delta_{\alpha}\right)}^{\mathbf{R}}\left(K_{t}\right) * F\right| \leq$ $c \Phi *|F|$, where $\Phi(x)=(1+|x|)^{-(1 / 2+\epsilon)}$. Since $\Phi$ belongs to $L^{1}(\mathbf{R})$, the right hand side of (80) is dominated by $\|F\|_{L^{1}(\mathbf{R})}$. This means that $M_{\mathbf{H}}^{\epsilon}, t>1$ and $\epsilon>1 / 2$, is bounded from $W_{-}\left(L^{1}(\mathbf{R})\right)$ to $L^{1}(G / / K)$. Since $\mathrm{H}_{\infty, 0}^{1,+}(G / / K)^{\sharp} \subset W_{-}\left(L^{1}(\mathbf{R})\right.$ ) (see (76)), the desired result follows.

Case II: $0<\epsilon \leq 1 / 2$. Since $t^{-\epsilon} W_{-(m+\xi)}^{\mathbf{R}}\left(K_{t}\right)$ is a convolution operator, to obtain the desired $L^{1}$-boundedness on $W_{-}\left(H_{\infty, 0}^{1,1-\epsilon}(\mathbf{R})\right)$, it is enough to prove that there exists a constant $C>0$ such that for each centered $(1, \infty, 0,1-\epsilon)$-atom $A$ on $\mathbf{R}, 0 \leq m \leq \underline{n}$, and $\xi \in \underline{D}$,

$$
\left\|\sup _{t>1} t^{-\epsilon}\left|W_{-(m+\xi)}^{\mathbf{R}}\left(K_{t}\right) * A\right|\right\|_{L^{1}(\mathbf{R})} \leq C .
$$

Let $[-r, r]$ denote the support of $A$ and, for simplicity, we put

$$
K_{t, m+\xi}^{\epsilon}(x)=t^{-\epsilon} W_{-(m+\xi)}^{\mathbf{R}}\left(K_{t}\right)(x) .
$$

First, we shall prove that

$$
K_{t, m+\xi}^{\epsilon}(x) \leq c\left(1+|x|^{1 / 2-\epsilon}\right) \Psi_{t}(x)
$$

where $\Psi_{t}$ is a Euclidean dilation of $\Psi(x)=(1+|x-2 \rho|)^{-2}$. Actually, if $\rho t \geq 2|x|$, then $(|x|-2 \rho t)^{2}=|x|^{2}+4 \rho t(\rho t-|x|) \geq|x|^{2}+2 \rho^{2} t^{2}$ and thus, it follows from (78), (79) that $K_{t}(x) \leq e^{-\left(|x|^{2}+2 \rho^{2} t^{2}\right) / 4 t} \leq e^{-(|x| / t)^{2}} e^{-\rho^{2} t / 2} \leq c t^{-1}(1+|x| / t)^{-2}$, because $t \geq 1$. Thereby, the above estimate is clear. If $\rho t \leq 2|x|$, then, by letting $\gamma=2$ in Lemma 7.5 , it follows that $\left|K_{t, m+\xi}^{\epsilon}(x)\right| \leq c t^{-(\epsilon+1 / 2)}(1+|| x|/ t-2 \rho|)^{-2} \leq$ $c t^{-(\epsilon-1 / 2)} t^{-1}(1+|| x|/ t-2 \rho|)^{-2} \leq c x^{-(\epsilon-1 / 2)} t^{-1}(1+|| x|/ t-2 \rho|)^{-2}$ for all $0 \leq m \leq \underline{n}$ and $\xi \in \underline{D}$. Hence we have the desired estimate. By using this estimate, we see that

$$
\begin{aligned}
\left|K_{t, m+\xi}^{\epsilon} * A(x)\right| & \leq c \int_{x-r}^{x+r}\left(1+|y|^{1 / 2-\epsilon}\right) \Psi_{t}(y)|A(x-y)| d y \\
& \leq c\left(1+|x+r|^{1 / 2-\epsilon}\right) \Psi_{t} *|A|(x) \\
& \leq c\left(1+|x+r|^{1 / 2-\epsilon}\right) M_{\Psi}^{\mathbf{R}}(|A|)(x),
\end{aligned}
$$

where $M_{\Psi}^{\mathbf{R}}(F)=\sup _{0<t<\infty}\left|\Psi_{t} * F(x)\right|$. Since $M_{\Psi}^{\mathbf{R}}$ is bounded on $L^{\infty}(\mathbf{R})$ and 
$\|A\|_{\infty} \leq r^{-1}(1+r)^{-(1-\epsilon)}$, it follows that, on $|x| \leq 2 r$

$$
\begin{aligned}
\int_{|x| \leq 2 r} \sup _{t>1}\left|K_{t, m+\xi}^{\epsilon} * A(x)\right| d x & \leq c\left(1+r^{1 / 2-\epsilon}\right) \int_{|x| \leq 2 r} M_{\Psi}^{\mathbf{R}}(|A|)(x) d x \\
& \leq c\left(1+r^{1 / 2-\epsilon}\right) r\|A\|_{\infty} \\
& \leq c\left(1+r^{1 / 2-\epsilon}\right)(1+r)^{-(1-\epsilon)} \leq C
\end{aligned}
$$

On the other hand, on $|x|>2 r$, it follows from the moment condition of $A$ that

$$
\begin{aligned}
K_{t, m+\xi}^{\epsilon} * A(x) & =\int_{-\infty}^{\infty} K_{t, m+\xi}^{\epsilon}(y) A(x-y) d y \\
& =\int_{x-r}^{x+r}\left(K_{t, m+\xi}^{\epsilon}\right)^{\prime}(y)\left(\int_{-\infty}^{y} A(x-s) d s\right) d y
\end{aligned}
$$

Lemma 7.5 with $\gamma=1+\epsilon, \epsilon>0$, and $\mu=1$ yields that $\left|\left(K_{t, m+\xi}^{\epsilon}\right)^{\prime}(y)\right| \leq c t^{-(1+\epsilon)}(1+$ $\| y|/ t-2 \rho|)^{-(1+\epsilon)} \leq c(1+|y|)^{-(1+\epsilon)}$. Since $\|A\|_{1} \leq r\|A\|_{\infty} \leq(1+r)^{-(1-\epsilon)}$, it follows that

$$
\sup _{t>1}\left|K_{t, m+\xi}^{\epsilon} * A(x)\right| \leq c(1+|x-r|)^{-(1+\epsilon)} \cdot r \cdot(1+r)^{-(1-\epsilon)}
$$

Therefore,

$$
\int_{|x| \geq 2 r} \sup _{t>1}\left|K_{t, m+\xi}^{\epsilon} * A(x)\right| d x \leq \int_{|x| \geq 2 r} \frac{(1+r)^{\epsilon}}{(1+|x-r|)^{1+\epsilon}} d x \leq C .
$$

Hence we see that $\left\|\sup _{t>1} K_{t, m+\xi}^{\epsilon} * A\right\|_{L^{1}(\mathbf{R})} \leq C$.

Case III: $\epsilon=0$. Let $A$ be a centered $(1, \infty, 1,3 / 2)$-atom on $\mathbf{R}$. On $|x| \leq 2 r$, similarly as for (82), we see that

$$
\int_{|x| \leq 2 r} \sup _{t>1}\left|K_{t, m+\xi}^{0} * A(x)\right| d x \leq C .
$$

Moreover, the moment condition of $A$ yields that $\int_{-\infty}^{y} A(x-s) d s$ is supported on $[x-r, x+r]$ and

$$
\int_{-\infty}^{\infty}\left(\int_{-\infty}^{t} A(x-s) d s\right) d t=0
$$

Then

$$
K_{t, m+\xi}^{0} * A(x)=\int_{x-r}^{x+r}\left(K_{t, m+\xi}^{0}\right)^{\prime \prime}(y)\left(\int_{-\infty}^{y}\left(\int_{-\infty}^{t} A(x-s) d s\right) d t\right) d y
$$


Since $\|A\|_{\infty} \leq r^{-1}(1+r)^{-3 / 2}$, Lemma 7.5 with $\gamma=3 / 2$ and $\mu=2$ similarly yields that

$$
\left|K_{t, m+\xi}^{0} * A(x)\right| \leq c(1+|x-r|)^{-3 / 2} r \cdot r \cdot(1+r)^{-3 / 2} .
$$

Therefore, on $|x| \geq 2 r$, it follows that

$$
\int_{|x| \geq 2 r} \sup _{t>1}\left|K_{t, m+\xi}^{0} * A(x)\right| d x \leq \int_{|x| \geq 2 r} \frac{(1+r)^{1 / 2}}{(1+|x-r|)^{3 / 2}} d x \leq C .
$$

Hence we see that $\left\|\sup _{t>1} K_{t, m+\xi}^{0} * A\right\|_{L^{1}(\mathbf{R})} \leq C$.

REMARK 7.6. Let $\epsilon=1 / 2$. Then $M_{\mathbf{H}}^{1 / 2}$ is bounded from $\mathrm{H}_{\infty, 0}^{1,+}(G / / K)^{\sharp} \cap$ $W_{-}\left(H_{\infty, 0}^{1,1 / 2}(\mathbf{R})\right)$ to $L^{1}(G / / K)$ by Theorem 7.4. In the proof we use a $(1, \infty, 0,1 / 2)$ atom $A$, whose $L^{\infty}$-norm has a decay $r^{-1}(1+r)^{-1 / 2}$. This extra decay $(1+r)^{-1 / 2}$ is only used to deduce (83). Clearly, if the first derivative of $K_{t}$ creates a decay $t^{-1}$, same as the one for $\Psi_{t}$ of a Euclidean dilation of $\Psi$, then we do not need use the extra decay. Actually, we may apply a common argument used to prove that a radial maximal operator $M_{\Phi}$ on $\mathbf{R}$ is bounded from $H_{\infty, 0}^{1}(\mathbf{R})$ to $L^{1}(\mathbf{R})$ (cf. [4, $\S 3])$. However, as shown in Lemma 7.5, the first derivative of $K_{t}$ creates only $t^{-1 / 2}$ decay. Therefore, we need the modification of the $L^{\infty}$-norm of $A$. This situation is the same in the case of $\epsilon=0$. The second derivative of $K_{t}$ creates a decay $t^{-1}$, not $t^{-2}$ (see Lemma 7.5) and thus, we need an extra decay $(1+r)^{-3 / 2}$ (see (84)).

Theorem 7.7. $M_{\mathbf{P}}$ is bounded from $H^{1}(G / / K)$ to $L^{1}(G / / K)$.

Proof. Since $M_{\mathbf{P}}^{\text {loc }}$ is bounded from $H_{\infty, 0}^{1,+}(G / / K)^{\sharp}$ to $L^{1}(G / / K)$, we may suppose that $t>1$ in the definition of $M_{\mathbf{P}}$ (see Theorem 6.3). Let $M_{\mathbf{P}, \gamma}^{\mathbf{R}}, \gamma \in \mathbf{R}$, denote the maximal operator on $\mathbf{R}$ associated to the Fourier multiplier corresponding to $\hat{p}_{t}(\lambda+i \rho)(i \lambda)^{\gamma}=W_{+}\left(p_{t}\right)^{\sim}(\lambda)(i \lambda)^{\gamma}$ :

$$
M_{\mathbf{P}, \gamma}^{\mathbf{R}}(F)=\sup _{t>1}\left|\int_{-\infty}^{\infty} \hat{p}_{t}(\lambda+i \rho)(i \lambda)^{\gamma} F^{\sim}(\lambda) e^{i \lambda x} d \lambda\right| .
$$

Then for $f \in H^{1}(G / / K)$, we have

$$
\begin{aligned}
\left\|M_{\mathbf{P}} f\right\|_{L^{1}(G)} & \leq c \sum_{m=0}^{\underline{n}} \sum_{\xi \in \underline{D}}\left\|M_{\mathbf{P}, 0}^{\mathbf{R}} \circ W_{-(m+\xi)}^{\mathbf{R}}(F)\right\|_{L^{1}(\mathbf{R})} \\
& \leq c \sum_{m=0}^{\underline{n}} \sum_{\xi \in \underline{D}}\left\|M_{\mathbf{P}, m+\xi}^{\mathbf{R}}(F)\right\|_{L^{1}(\mathbf{R})},
\end{aligned}
$$


where $F=W_{+}(f)$. Since $F \in W_{-}\left(H^{1}(\mathbf{R})\right)$ (see Theorem 6.3$), F$ has a $(1, \infty, 2)$ atomic decomposition $F=\sum_{i} \lambda_{i} A_{i}$. Hence, it is enough to show that $\left\|M_{\mathbf{P}, m+\xi}^{\mathbf{R}}(A)\right\|_{L^{1}(\mathbf{R})} \leq c$ for all $(1, \infty, 2)$-atom $A$. Clearly, we may suppose that $A$ is centered, that is, supported in $[-r, r]$. We recall that

$$
\hat{p}_{t}(\lambda+1 \rho)=K_{t}^{\sim}(\lambda), \quad K_{t}(x)=\frac{c t}{\left(t^{2}+x^{2}\right)^{1 / 2}} \mathrm{~K}_{t}\left(\rho\left(t^{2}+x^{2}\right)^{1 / 2}\right) e^{\rho x},
$$

where $\mathrm{K}_{t}$ is the modified Bessel function (see $[1, \S 6]$ ).

LemMA 7.8. Let notations be as above and $M_{H L}^{\mathrm{R}}$ be the Hardy-Littlewood maximal function on $\mathbf{R}$. Then for all $F \in L^{1}(\mathbf{R})$ and $\gamma \geq 0$,

$$
M_{\mathbf{P}, \gamma}^{\mathbf{R}}(F)(x) \leq c M_{H L}^{\mathbf{R}}(F)(x) .
$$

In particular, $M_{\mathbf{P}, \gamma}^{\mathbf{R}}$ satisfies the maximal theorem.

Proof. We note that $\left|W_{\gamma}^{\mathbf{R}} K_{t}(x)\right| \leq c K_{t}(x)$ (see [1, p. 289]) and $\mathrm{K}_{t}(x) \leq$ $c|x|^{-1 / 2} e^{-t|x|}$. Hence we see that

$$
M_{\mathbf{P}, \gamma}^{\mathbf{R}}(F)(x) \leq c \int_{-\infty}^{\infty}|F(y)| \frac{t}{\left(t^{2}+(x-y)^{2}\right)^{3 / 4}} e^{-\rho\left(t^{2}+(x-y)^{2}\right)^{1 / 2}} e^{\rho|x-y|} d y .
$$

Here we divide the integral as $\int_{I_{0}}+\sum_{k=1}^{\infty} \int_{I_{k}}$, where $I_{0}=\left\{y ;|x-y| \leq t^{2}\right\}$ and $I_{k}=\left\{y ; 2^{k} t^{2}<|x-y| \leq 2^{k+1} t^{2}\right\}, k=1,2, \cdots$. Since $t^{3} e^{-\rho\left(t^{2}+x^{2}\right)^{1 / 2}}$ has the maximum at $t \sim\left(t^{2}+x^{2}\right)^{1 / 4}$, it follows that

$$
\begin{aligned}
& \int_{I_{0}}|F(y)| \frac{t}{\left(t^{2}+(x-y)^{2}\right)^{3 / 4}} e^{-\rho\left(t^{2}+(x-y)^{2}\right)^{1 / 2}} e^{\rho|x-y|} d y \\
\leq & c \frac{1}{t^{2}} \int_{|x-y| \leq t^{2}}|F(y)| d y \leq c M_{H L}^{\mathbf{R}}(F)(x) .
\end{aligned}
$$

Moreover,

$$
\begin{aligned}
& \int_{I_{k}}|F(y)| \frac{t}{\left(t^{2}+(x-y)^{2}\right)^{3 / 4}} e^{-\rho\left(t^{2}+(x-y)^{2}\right)^{1 / 2}} e^{\rho|x-y|} d y \\
\leq & c \int_{|x-y| \leq 2^{k+1} t^{2}}|F(y)| \frac{t}{\left(2^{k} t^{2}\right)^{3 / 2}} d y \\
\leq & c 2^{-k / 2} \frac{1}{2^{k+1} t^{2}} \int_{|x-y| \leq 2^{k+1} t^{2}}|F(y)| d y \\
\leq & c 2^{-k / 2} M_{H L}^{\mathrm{R}}(F)(x) .
\end{aligned}
$$

Hence, the desired result follows. 
Lemma 7.9. Let $A$ be $a(1, \infty, 2)$-atom on $\mathbf{R}$ supported on $[-r, r]$. Then for $x \geq 2 r$,

$$
M_{\mathbf{P}, \gamma}^{\mathbf{R}}(A)(x) \leq c \max \left(\frac{r^{1 / 2}}{|x-r|^{3 / 2}}, \frac{r}{x^{2}}\right)
$$

Proof. Since $\|A\|_{L^{1}(\mathbf{R})} \leq 1$ and $|x-y| \geq|x-r|$ if $|y| \leq r$, it follows that

$$
M_{\mathbf{P}, \gamma}^{\mathbf{R}}(A)(x) \leq c \int_{-\infty}^{\infty}|A(y)| \frac{t}{\left(t^{2}+(x-y)^{2}\right)^{3 / 4}} d y \leq c \frac{t}{|x-r|^{3 / 2}}
$$

Especially,

$$
M_{\mathbf{P}, \gamma}^{\mathbf{R}}(A)(x) \leq c \frac{r^{1 / 2}}{|x-r|^{3 / 2}} \quad \text { if } \quad r \geq t^{2} .
$$

On the other hand, we note that

$$
A * W_{\gamma}^{\mathbf{R}}\left(K_{t}\right)(x)=c \int_{-\infty}^{\infty} \hat{p}_{t}(\lambda+i \rho) \tilde{A}(\lambda) \lambda^{\gamma} e^{-i \lambda x} d \lambda .
$$

Since $\lambda(\lambda+2 i \rho)=|\lambda||\lambda+2 i \rho| e^{i \theta}, \tan \theta=2 \rho / \lambda$, and thus, $\left.\Re\left((\lambda+i \rho)^{2}+\rho^{2}\right)\right)^{1 / 2}=$ $|\lambda|^{1 / 2}|\lambda+2 i \rho|^{1 / 2} \cos (\theta / 2) \geq c|\lambda|^{1 / 2}$ with $c>0$, it is easy to see that

$$
\begin{aligned}
& \left|\hat{p}_{t}(\lambda+i \rho)\right| \leq c e^{-c t|\lambda|^{1 / 2}} \\
& \left|\left(\frac{d}{d \lambda}\right) \hat{p}_{t}(\lambda+i \rho)\right| \leq c t\left(\frac{1+|\lambda|}{|\lambda|}\right)^{1 / 2} e^{-c t|\lambda|^{1 / 2}} \\
& \left|\left(\frac{d}{d \lambda}\right)^{2} \hat{p}_{t}(\lambda+i \rho)\right| \leq c\left(t^{2} \frac{1+|\lambda|}{|\lambda|}+t \frac{1}{|\lambda|}\left(\frac{1+|\lambda|}{|\lambda|}\right)^{1 / 2}\right) e^{-c t|\lambda|^{1 / 2}}
\end{aligned}
$$

Since $A$ is a $(1, \infty, 2)$-atom on $\mathbf{R}$ supported on $[-r, r]$, it follows that $\left|(d / d \lambda)^{n} \hat{A}(\lambda)\right|$ $\leq r^{n}(n \in \mathbf{N})$ and $\left|(d / d \lambda)^{n} \hat{A}(\lambda)\right| \leq r^{n+1}|\lambda|, n=0,1,2$, by the moment condition of $A$. Moreover, since $(d / d \lambda)^{n-k} \hat{A}(\lambda)$ is the $k$-fold integral of $(d / d \lambda)^{n} \hat{A}(\lambda)$ over $[0, \lambda]$, it follows that $\left|(d / d \lambda)^{n-k} \hat{A}(\lambda)\right| \leq r^{n+1}|\lambda|^{k+1}, 0 \leq k \leq n=0,1,2$. Therefore,

$$
\begin{aligned}
& \left|x^{2} A * W_{\gamma}^{\mathbf{R}}\left(K_{t}\right)(x)\right| \\
= & c\left|\int_{-\infty}^{\infty} \frac{d^{2}}{d \lambda^{2}}\left(\hat{p}_{t}(\lambda+i \rho) \tilde{A}(\lambda) \lambda^{\gamma}\right) e^{-i \lambda x} d \lambda\right| \\
\leq & c \int_{-\infty}^{\infty}\left(\left(t^{2} \frac{1+|\lambda|}{|\lambda|}+t \frac{1}{|\lambda|}\left(\frac{1+|\lambda|}{|\lambda|}\right)^{1 / 2}\right) r^{2}|\lambda|^{\gamma+2}+r^{2}|\lambda|^{\gamma}\right) e^{-c t|\lambda|^{1 / 2}} d \lambda \\
\leq & c \frac{r^{2}}{t^{2+2 \gamma}} .
\end{aligned}
$$


This means that

$$
\left|A * W_{\gamma}^{\mathbf{R}}\left(K_{t}\right)(x)\right| \leq c \frac{r}{x^{2}} \frac{r}{t^{2+2 \gamma}}
$$

and thus, since $t \geq 1$, it follows that

$$
M_{\mathbf{P}, \gamma}^{\mathbf{R}}(A)(x) \leq c \frac{r}{x^{2}} \quad \text { if } \quad r \leq t^{2} .
$$

This completes the proof of the lemma.

We return to the proof of Theorem 7.7. Since $M_{\mathbf{P}, \gamma}^{\mathbf{R}}$ is bounded on $L^{2}(\mathbf{R})$ (see Lemma 7.8) and $\|A\|_{2} \leq r^{-1 / 2}$, it follows that

$$
\int_{|x| \leq 2 r} M_{\mathbf{P}, \gamma}^{\mathbf{R}}(A)(x) d x \leq r^{1 / 2}\|A\|_{2} \leq c .
$$

Moreover, it follows from Lemma 7.9 that

$$
\int_{|x|>2 r} M_{\mathbf{P}, \gamma}^{\mathbf{R}}(A)(x) d x \leq \int_{|x|>2 r}\left(\frac{r^{1 / 2}}{|x-r|^{3 / 2}}+\frac{r}{x^{2}}\right) d x \leq c .
$$

Hence we obtain that $\left\|M_{\mathbf{P}, \gamma}^{\mathbf{R}}(A)\right\|_{L^{1}(\mathbf{R})} \leq c$ for all $(1, \infty, 2)$-atom $A$.

We last treat the Riesz transform $\mathrm{R}$ on $G$. Under the standard notation in [14] we denote the covariant differentiation on $G$ by $\nabla:|\nabla|^{2}(f)=\Omega\left(f^{2}\right)-2 \Omega f \cdot f$ for $f \in C^{\infty}(G)$. Then the Riesz transform $\mathrm{R}$ on $G$ is defined as follows.

Definition 7.10. For $f \in C_{c}^{\infty}(G / / K)$

$$
(\mathrm{R} f)(g)=|\nabla| \circ(-\Omega)^{-1 / 2}(f)(g) \quad(g \in G) .
$$

This operator $\mathrm{R}$ also satisfies the maximal theorem (see [1, Corollary 5.2]). We recall that $|\nabla f|^{2}(g)=\sum_{m=1}^{n}\left|X_{i} f\right|^{2}(g)(g \in G)$, here $\left\{X_{i} ; 1 \leq i \leq n\right\}$ is denoted as an orthonormal basis of the Lie algebra $\mathfrak{g}$ of $G$ and each $X_{i}$ is regarded as a left (or right) invariant differential operator on $G$. Especially, since $f$ is $K$-bi-invariant on $G,|\nabla f|^{2}(g)$ is simply expressed as $c\left|(d / d x) f\left(a_{x}\right)\right|^{2}$ provided $\sigma(g)=x$ (cf. $[1, \S 2]$ ).

Now let $\ell(\lambda)=i \lambda / \sqrt{\lambda(\lambda+2 i \rho)}$ and $\mathrm{M}_{\ell}$ the corresponding Fourier multiplier. Then, since $(-\Omega)^{-1 / 2}$ is the Fourier multiplier corresponding to $\left(\lambda^{2}+\rho^{2}\right)^{-1 / 2}$, applying the similar argument used in the proof of Theorem 4.6, we can deduce that

$$
\|\mathrm{R} f\|_{L^{1}(G)} \leq c \sum_{m=0}^{\underline{n}} \sum_{\xi \in \underline{D}}\left\|\mathrm{M}_{\ell} \circ W_{-(m+\xi)}^{\mathbf{R}}(F)\right\|_{L^{1}(\mathbf{R})} .
$$

Since $\ell(\lambda)$ satisfies the Hörmander condition and $m+\xi \leq \alpha+1 / 2$, Remark 4.7 yields the following. 
Theorem 7.11. $\mathrm{R}$ is bounded from $W_{-}\left(\mathrm{M}_{C_{\rho}}\left(H^{1}(\mathbf{R})\right)\right)$ to $L^{1}(G / / K)$.

\section{Appendix}

A. Estimate of $\boldsymbol{\Gamma}_{\boldsymbol{m}}$ : In [8, Proposition 8.3] we estimate derivatives of $\Gamma_{m}(\lambda)$ when $\Im \lambda \geq \rho$ and show that $\Gamma_{m}(\xi+i \rho)(\xi \in \mathbf{R})$ satisfies Hörmander's condition. Here we shall obtain an estimate of $\Gamma_{m}(\lambda)$ for $\Im \lambda \geq-\rho$. We refer to the notations and the proof in [3, Lemma 7] and we denote $\Gamma_{2 m}$ by $\Gamma_{m}$. Then the recursive definition of $\Gamma_{m}(\lambda)$ yields that

$$
\left|\Gamma_{m}(\lambda)\right| \leq \frac{\rho|\rho-i \lambda|}{m|m-i \lambda|} \prod_{k=1}^{m-1}\left(1+\frac{r_{k}(\lambda)}{c_{k}(\lambda)}\right)
$$

Here

$$
c_{k}(\lambda)=4 k|k-i \lambda| \quad \text { and } \quad r_{k}(\lambda)=4 \kappa|2 k-i \lambda+\rho|,
$$

where $\kappa=\alpha-\beta$ if $k \equiv m+1(\bmod 2)$ and $\kappa=\rho$ if $k \equiv m(\bmod 2)$.

LEMMA 8.1. For each $\delta>0$, there exists a positive constant $c$ such that for all $m \in \mathbf{N}$ and $\lambda=\xi-i \rho(\xi \in \mathbf{R})$,

$$
\left|\Gamma_{m}(\lambda)\right| \leq c m^{2 \alpha+\delta}
$$

Proof. For each $\epsilon>0$ we take $n_{0} \in \mathbf{N}$ such that $4\left(\rho+n_{0}\right)^{2} / n_{0}^{2} \leq 4(1+\epsilon)^{2}$. We first estimate each $k^{2} \theta^{-2} r_{k}(\lambda)^{2} c_{k}(\lambda)^{-2}=|2 k-i \lambda+\rho|^{2} /|k-i \lambda|^{2}$.

If $k \geq \rho+n_{0}$, then

$$
\frac{|2 k+\rho-i \lambda|^{2}}{|k-i \lambda|^{2}}=\frac{4 k^{2}+\xi^{2}}{(k-\rho)^{2}+\xi^{2}} \leq \frac{4 k^{2}}{(k-\rho)^{2}} \leq \frac{4\left(\rho+n_{0}\right)^{2}}{n_{0}^{2}} \leq 4(1+\epsilon)^{2} .
$$

If $k<\rho+n_{0}$ and $\rho \notin \mathbf{N}$, then

$$
\frac{|2 k+\rho-i \lambda|^{2}}{|k-i \lambda|^{2}} \leq \frac{4 k^{2}}{(k-\rho)^{2}} \leq \frac{4\left(\rho+n_{0}\right)^{2}}{(1 / 2)^{2}} \leq c .
$$

If $k<\rho+n_{0}, \rho \in \mathbf{N}$, and $k \neq \rho$, then

$$
\frac{|2 k+\rho-i \lambda|^{2}}{|k-i \lambda|^{2}} \leq \frac{4 k^{2}}{(k-\rho)^{2}} \leq 4\left(\rho+n_{0}\right)^{2} \leq c .
$$

If $k<\rho+n_{0}$ and $k=\rho$, then

$$
\frac{|2 k+\rho-i \lambda|^{2}}{|k-i \lambda|^{2}}=\frac{4 \rho^{2}+\xi^{2}}{\xi^{2}} \leq c\left(\frac{1+|\xi|}{|\xi|}\right)^{2}
$$


Therefore, substituting these estimates into (85), we have the following.

Case I. $\rho \notin \mathbf{N}$ : Since

$$
\left(k \frac{r_{k}(\lambda)}{c_{k}(\lambda)}\right)^{2} \leq \begin{cases}(2(1+\epsilon) \theta)^{2} & \text { if } k \geq \rho+n_{0} \\ c & \text { if } k<\rho+n_{0}\end{cases}
$$

it follows that, if $\rho+n_{0} \leq m-1$, then

$$
\begin{aligned}
& \prod_{k=1}^{m-1}\left(1+\frac{r_{k}(\lambda)}{c_{k}(\lambda)}\right) \\
\leq & \exp \left(\sum_{k=1}^{m-1} \frac{r_{k}(\lambda)}{c_{k}(\lambda)}\right)=\exp \left(\sum_{k=1}^{\rho+n_{0}-1} \frac{r_{k}(\lambda)}{c_{k}(\lambda)}+\sum_{k=\rho+n_{0}}^{m-1} \frac{r_{k}(\lambda)}{c_{k}(\lambda)}\right) \\
\leq & \exp \left(c\left(\rho+n_{0}-1\right)+\rho \sum_{\substack{k \equiv m \\
1 \leq k \leq m-1}} 2(1+\epsilon) k^{-1}+(\alpha-\beta) \sum_{\substack{k \equiv m+1 \\
1 \leq k \leq m-1}} 2(1+\epsilon) k^{-1}\right) \\
\leq & c \exp ((1+\epsilon)(\rho+\alpha-\beta) \log m) \leq c m^{(1+\epsilon)(2 \alpha+1)} .
\end{aligned}
$$

Therefore, we see that

$$
\left|\Gamma_{m}(\lambda)\right| \leq b_{m}(\lambda) \leq c \frac{\rho}{m} \cdot \frac{|\xi|}{|m-\rho|+|\xi|} \cdot m^{(1+\epsilon)(2 \alpha+1)} \leq c m^{(1+\epsilon)(2 \alpha+1)-1} .
$$

Clearly, if $\rho+n_{0}>m-1$, then $\left|\Gamma_{m}(\lambda)\right| \leq c$.

Case II. $\rho \in \mathbf{N}$ : In this case there is a possibility that the term corresponding to $k=\rho$ will appear in the product $\prod_{k=1}^{m-1}$ when $\rho \leq m-1$. Therefore, the above estimate is changed as

$$
\begin{aligned}
\left|\Gamma_{m}(\lambda)\right| & \leq c \frac{\rho}{m} \cdot \frac{|\xi|}{|m-\rho|+|\xi|} \cdot\left(1+\frac{1+|\xi|}{|\xi|}\right) \cdot m^{(1+\epsilon)(2 \alpha+1)-1} \\
& \leq c m^{(1+\epsilon)(2 \alpha+1)-1} .
\end{aligned}
$$

In both cases, letting $\epsilon$ sufficiently small so as to satisfy $(1+\epsilon)(2 \alpha+1)-1 \leq$ $2 \alpha+\delta$, we can obtain the desired estimate.

Let $k \in \mathbf{N}$ and $k \neq \rho$ if $\rho \in \mathbf{N}$. Applying the above argument for $\lambda=\xi-i k$ $(\xi \in \mathbf{R})$, we see that each $\Gamma_{m}(\lambda), k \leq m-1$, has a pole at $\lambda=-i k$. On the other hand, if $\Im \lambda \neq-k$, we can deduce the same estimate. Therefore, the estimate obtained in Lemma 8.1 also holds on the tube domain $\mathrm{F}(a, b)=\{\lambda \in \mathbf{C} ; a<\Im \lambda<$ $b\}$ where $[a, b]$ does not contain $-k(k \in \mathbf{N})$ except $-\rho$ if $\rho \in \mathbf{N}$. Hence, Cauchy's integral formula yields the following. 
Proposition 8.2. Suppose that the real interval $[a, b]$ does not contain $-k$ $(k \in \mathbf{N})$ except $-\rho$ if $\rho \in \mathbf{N}$. Then for each $\delta>0$ and $n \in \mathbf{N}$, there exists a positive constant $c$ such that for all $m \in \mathbf{N}$ and $\lambda \in \mathrm{F}(a, b)$,

$$
\left|\left(\frac{d}{d \lambda}\right)^{n} \Gamma_{m}(\lambda)\right| \leq c m^{2 \alpha+\delta}
$$

REMARK 8.3. When $\Im \lambda \geq \rho$, we can replace $4(1+\epsilon)^{2}$ in (86) by 4 . Thereby, we can replace the power $2 \alpha+\delta$ in (87) by $2 \alpha$ (see [8, Proposition 8.3]).

B. Maximal functions on R: In this section $\phi$ denotes a compactly supported $C^{\infty}$ function on $\mathbf{R}$ with $\int_{-\infty}^{\infty} \phi(x) d x=1$, and $\phi_{t}$ a Euclidean dilation:

$$
\phi_{t}(x)=\frac{1}{t} \phi\left(\frac{x}{t}\right), \quad t>0 .
$$

We define the radial maximal function $M_{\phi}(F)$ on $\mathbf{R}$ as

$$
\left(M_{\phi} F\right)(x)=\sup _{0<t<\infty}\left|F * \phi_{t}(x)\right| .
$$

Then $F \in L_{\text {loc }}^{1}(\mathbf{R})$ belongs to $H^{1}(\mathbf{R})$, by the definition, if $M_{\phi} F$ belongs to $L^{1}(\mathbf{R})$ (cf. [15, p. 87]). We shall consider another characterization of $H^{1}(\mathbf{R})$ by using a maximal function associated to a Fourier multiplier $\mathbf{M}_{m(t, \cdot)}$ corresponding to a function $m(t, \lambda)$ on $\mathbf{R}_{+} \times \mathbf{R}$ :

$$
\mathrm{M}_{m(t, \cdot)}(F)^{\sim}(\lambda)=m(t, \lambda) F^{\sim}(\lambda)
$$

Definition 8.4. We say that $m(t, \lambda)$ is in the class $\mathrm{A}_{N, M}(N, M \in \mathbf{N})$ if there exists a constant $c$ such that

$$
\left|\left(\frac{d}{d \lambda}\right)^{n} m(t, \lambda)\right| \leq c t^{n}(1+|t \lambda|)^{-M} \quad(0 \leq n \leq N) .
$$

We define related maximal operators $M_{m}, M_{\mathrm{A}_{N, M}}, M_{m, L}^{* *}(L \geq 0)$ and $M_{m}^{*}$ on $\mathbf{R}$ as follows.

Definition 8.5. Let $N, M, L \in \mathbf{N}$ and suppose that $M>1$. For $F \in$ $L_{\text {loc }}^{1}(\mathbf{R})$ and $m(t, \lambda) \in \mathrm{A}_{N, M}$, we define

$$
\begin{aligned}
M_{m} F(x) & =\sup _{0<t<\infty}\left|\mathrm{M}_{m(t, \cdot)} F(x)\right|, \\
M_{\mathrm{A}_{N, M}} F(x) & =\sup _{m \in \mathrm{A}_{N, M}} M_{m} F(x), \\
M_{m, L}^{* *} F(x) & =\sup _{0<t<\infty} \sup _{y \in \mathbf{R}}\left|F * m_{t}(x-y)\right|\left(1+\frac{|y|}{t}\right)^{-L},
\end{aligned}
$$




$$
M_{m}^{*} F(x)=\sup _{|x-y|<t}\left|F * m_{t}(y)\right|
$$

where $m_{t}(x)$ is the inverse Fourier transform of $m(t, \lambda)$.

Clearly, since $M>1, m_{t}(x)$ is well-defined and $M_{m} F(x) \leq M_{m}^{*} F(x) \leq$ $2 L M_{m, L}^{* *} F(x)$ holds pointwise.

Theorem 8.6. Let $M, N \geq 4$. Let $\ell(t, \lambda) \in \mathrm{A}_{N, M}$ and suppose that there exists $c>0$ such that

$$
|\ell(t, \lambda)| \geq c|t \lambda| \quad(0 \leq|t \lambda| \leq 2) .
$$

Then $\|F\|_{H^{1}(\mathbf{R})} \approx\left\|M_{\ell} F\right\|_{L^{1}(\mathbf{R})}$.

Proof. $\left\|M_{\ell} F\right\|_{L^{1}(\mathbf{R})} \leq c\|F\|_{H^{1}(\mathbf{R})}$ : By using the atomic characterization of $H^{1}(\mathbf{R})$, it is enough to show that $\left\|M_{\ell} A\right\|_{L^{1}(\mathbf{R})} \leq C$ for all $(1, \infty, 0)$-atom $A$ on $\mathbf{R}$. We may suppose that $A$ is supported on $[-r, r]$. We first note that, since $\ell(t, \lambda) \in \mathrm{A}_{N, M}$ with $M \geq 4$, the inverse Fourier transform of $\ell(t, \lambda)$ is dominated by $t^{-1}\left(1+|x / t|^{2}\right)^{-1}$ and hence, $M_{\ell}$ satisfies the maximal theorem (cf. [8, Lemma 5.1]). Next we note that the Fourier transform $A^{\sim}(\lambda)$ satisfies

$$
\left|\left(\frac{d}{d \lambda}\right)^{\ell} A^{\sim}(\lambda)\right| \leq r^{\ell} \quad(\ell \in \mathbf{N})
$$

and

$$
\left|A^{\sim}(\lambda)\right|=\left|\int_{0}^{\lambda} A^{\prime}(s) d s\right| \leq c r|\lambda|,
$$

because $A^{\sim}(0)=0$ by the moment condition. Therefore, it follows that

$$
\begin{aligned}
\left|x^{2} M_{\ell(t, \cdot)}(A)\right| & =\left|\int_{-\infty}^{\infty} A^{\sim}(\lambda) \ell(t, \lambda) \cdot\left(\frac{d}{d \lambda}\right)^{2} e^{i \lambda x} d \lambda\right| \\
& \leq \int_{-\infty}^{\infty}\left|\left(\frac{d}{d \lambda}\right)^{2}\left(A^{\sim}(\lambda) \ell(t, \lambda)\right)\right| d \lambda \\
& \leq c\left|M_{\ell(t, \cdot)}\left(x^{2} A\right)\right|+c \int_{-\infty}^{\infty} \frac{r t+r|\lambda| t^{2}}{(1+|t \lambda|)^{4}} d \lambda \\
& \leq c r
\end{aligned}
$$

because $\left\|M_{\ell(t, \cdot)}\left(x^{2} A\right)\right\|_{\infty} \leq c\left\|x^{2} A\right\|_{\infty} \leq c r$. This means that $\left|M_{\ell(t, \cdot)}(A)(x)\right| \leq$ $r|x|^{-2}$. Finally, we can deduce that

$$
\left\|M_{\ell}(A)\right\|_{L^{1}(\mathbf{R})} \leq c \int_{|x|<2 r}\|A\|_{\infty} d x+c \int_{|x| \geq 2 r} \frac{r}{|x|^{2}} d x \leq C .
$$


$\|F\|_{H^{1}(\mathbf{R})} \leq c\left\|M_{\ell} F\right\|_{L^{1}(\mathbf{R})}$ : The proof is quite similar as the one for $[15$, Theorem 1 in Chap. 3]. We shall give an outline for a necessary modification of the proof.

Step 1. $\|F\|_{H^{1}(\mathbf{R})} \leq c\left\|M_{\mathrm{A}_{N, M}} F\right\|_{L^{1}(\mathbf{R})}$ : Let $\psi \in C_{c}^{\infty}(\mathbf{R})$ and $\int_{-\infty}^{\infty} \psi(x) d x=$ 1. Since $A_{N, M}$ contains a function $m(t, \lambda)=\hat{\psi}_{t}(\lambda)=\hat{\psi}(t \lambda)$, the desired result follows from [15, Theorem 1 in Chap. 3].

Step 2. $M_{\mathrm{A}_{N, M}} F(x) \leq c M_{\ell, L}^{* *} F(x)$ : We refer to [15, Lemma 2 in p. 93 and 1.4 in p. 95]. We take $L \in \mathbf{N}$ such that $M, N>L+1$ and $L>1$. Let $\psi_{0} \in S(\mathbf{R})$ satisfy $\hat{\psi}_{0}(\lambda)=1$ if $|\lambda| \leq 1$ and $\hat{\psi}_{0}(\lambda)=0$ if $|\lambda| \geq 2$. Then there exists a decomposition of 1 of the form: $1=\sum_{k=0}^{\infty} \hat{\psi}_{k}(\lambda)$, where each $\hat{\psi}_{k}(k>0)$ is supported on $2^{k-1} \leq$ $|\lambda| \leq 2^{k+1}$ and $\left|(d / d \lambda)^{n} \hat{\psi}_{k}(\lambda)\right| \leq c 2^{-k n}$. For each $m(t, \lambda) \in \mathrm{A}_{N, M}$ we let

$$
\begin{aligned}
m(t, \lambda) & =\sum_{k=0}^{\infty} \hat{\psi}_{k}(t \lambda) m(t, \lambda) \\
& =\sum_{k=0}^{\infty} \frac{\hat{\psi}_{k}(t \lambda) m(t, \lambda)}{\ell\left(2^{-k} t, \lambda\right)} \cdot \ell\left(2^{-k} t, \lambda\right) \\
& =\sum_{k=0}^{\infty} \eta_{k}(t, \lambda) \ell\left(2^{-k} t, \lambda\right)
\end{aligned}
$$

By the assumption (90) it follows that $\ell\left(2^{-k} t, \lambda\right) \geq c 2^{-k}|t \lambda| \geq c 2^{-1}$ if $t \lambda \in$ $\left[2^{k-1}, 2^{k+1}\right]$ and moreover, $\left|(d / d \lambda)^{n} \ell(t, \lambda)\right| \leq c t^{n}(0 \leq n \leq N)$. Thereby, we easily see that

$$
\left|\left(\frac{d}{d \lambda}\right)^{n} \eta_{k}(t, \lambda)\right| \leq c t^{n}(1+|t \lambda|)^{-M}
$$

and, if we denote the inverse Fourier transform of $\eta_{k}(t, \lambda)$ by $\eta_{k, t}(y)$, then

$$
\begin{aligned}
\left|y^{n} \eta_{k, t}(y)\right| & \leq\left|\int_{2^{k-1} / t}^{2^{k+1} / t} \eta_{k}(t, \lambda) \cdot\left(\frac{d}{d \lambda}\right)^{n} e^{i \lambda y} d \lambda\right| \\
& \leq c t^{n} \int_{2^{k-1} / t}^{2^{k+1} / t}(1+|t \lambda|)^{-M} d \lambda \\
& \leq c t^{n-1} 2^{-k(M-1)}
\end{aligned}
$$

Since $N, M>L+1$, it follows that

$$
\begin{aligned}
\left(M_{m} F\right)(x) & \leq \sup _{t>0} \sum_{k=0}^{\infty}\left|\left(F * \eta_{k, t} * \ell_{2^{-k} t}\right)(x)\right| \\
& \leq \sup _{t>0} \sum_{k=0}^{\infty} \int_{-\infty}^{\infty}\left|F * \ell_{2^{-k} t}(x-y)\right|\left|\eta_{k, t}(y)\right| d y
\end{aligned}
$$




$$
\begin{aligned}
& \leq c M_{\ell, L}^{* *} F(x) \cdot \sup _{0<t<\infty} \sum_{k=0}^{\infty} \int_{-\infty}^{\infty}\left(1+\frac{|y|}{2^{-k} t}\right)^{L}\left(1+\frac{|y|}{t}\right)^{-N} \\
& \times t^{-1} \cdot 2^{-k(M-1)} d y \\
& \leq c M_{\ell, L}^{* *} F(x) \cdot \int_{-\infty}^{\infty}(1+|y|)^{L-N} d y \cdot \sum_{k=0}^{\infty} 2^{-k(M-L-1)} \\
& \leq c M_{\ell, L}^{* *} F(x) .
\end{aligned}
$$

Step 3. $\left\|M_{\ell, L}^{* *} F\right\|_{L^{1}(\mathbf{R})} \leq c\left\|M_{\ell}^{*} F\right\|_{L^{1}(\mathbf{R})}$ : See [15, Lemma 1 in p. 93].

Step 4. $\left\|M_{\ell}^{*} F\right\|_{L^{1}(\mathbf{R})} \leq c\left\|M_{\ell} F\right\|_{L^{1}(\mathbf{R})}$ : We refer to $[15,1.5$ in p. 95]. We put $f(x, t)=F * \ell_{t}(x)$ where $\ell_{t}(x)$ is the inverse Fourier transform of $\ell(t, \lambda)$. Then, for $\left|x^{\prime}-y\right| \leq r t$, there exists $x^{\prime}<z<y$ such that

$$
\left|f\left(x^{\prime}, t\right)-f(y, t)\right| \leq r t\left|\left(\frac{d}{d x}\right) f(z, t)\right|
$$

We here note that $t(d / d x) f=F *\left(t(d / d x) \ell_{t}\right)$ and $\left(t(d / d x) \ell_{t}\right)^{\wedge}(\lambda)=\ell(t, \lambda) \cdot(i t \lambda)$. Therefore, $\left(t(d / d x) \ell_{t}\right)^{\wedge}$ belongs to $A_{N, M-1}$. The rest of the proof is same as in [15] if we replace $S_{F}$ by $\mathrm{A}_{L, M}$.

These four steps complete the proof of the desired inequality.

C. Dual spaces of $\boldsymbol{W}_{-}\left(\boldsymbol{H}^{\mathbf{1}}(\mathbf{R})\right)$ : We shall introduce a dual space of $W_{-}\left(H^{1}(\mathbf{R})\right)$. For $f \in C_{c}^{\infty}(G / / K)$ we define the complex Fourier-Jacobi transform $\tilde{f}$ as

$$
\tilde{f}(\lambda)=\int_{0}^{\infty} f(x) \Phi(\lambda, x) \Delta(x) d x
$$

(see (7), $[10,(2)])$. Then for real valued functions $f, g \in C_{c}^{\infty}(G / / K)$, we can deduce the following Plancherel formula (cf. [10, Theorem 5.1]):

$$
\langle f, g\rangle_{L^{2}\left(\mathbf{R}_{+}, \Delta d x\right)}=c \int_{-\infty}^{\infty} \hat{f}(\lambda+i \rho) \tilde{g}(\lambda+i \rho) C(-\lambda-i \rho)^{-1} d \lambda .
$$

We suppose that $f \in W_{-}\left(H^{1}(\mathbf{R})\right)$. Since $\hat{f}(\lambda+i \rho)=W_{+}(f)^{\sim}(\lambda)$ (see $\left.(21)\right)$ and $W_{+}(f) \in H^{1}(\mathbf{R})$ by the definition, a dual space of $W_{-}\left(H^{1}(\mathbf{R})\right)$ should be defined as the space consisting of $g$ such that $\tilde{g}(\lambda+i \rho) C(-\lambda-i \rho)^{-1}$ is the Fourier transform of a function in the dual space of $H^{1}(\mathbf{R})$, that is, $B M O(\mathbf{R})$ (cf. [15, Chap. 4]). We shall paraphrase this definition by using fractional calculus on $G$. First we introduce a generalized Riemann-Liouville type fractional integral operator $\tilde{W}_{\mu}^{\sigma}$ : For $\sigma>0$ and $\mu \in \mathbf{C}, \tilde{W}_{\mu}^{\sigma}(F)(y), y>0$, is defined by

$$
\tilde{W}_{\mu}^{\sigma}(f)(y)=\frac{\sigma^{-1}}{\Gamma(\mu+n)} \frac{d^{n}}{d(\operatorname{ch} \sigma y)^{n}} \int_{0}^{y} f(x)(\operatorname{ch} \sigma y-\operatorname{ch} \sigma x)^{\mu+n-1} d x \cdot \operatorname{sh} \sigma y
$$


where $n=0$ if $\Re \mu>0$ and $-n<\Re \mu \leq-n+1, n=0,1,2, \cdots$, if $\Re \mu \leq 0$. Similarly as in (18), if we set

$$
\tilde{W}_{+}(f)=\tilde{W}_{\beta+1 / 2}^{2} \circ \tilde{W}_{\alpha-\beta}^{1}\left(e^{\rho x} f\right)
$$

then we see from $[11, \S 3]$ and $[10$, Theorem 5.1] that

$$
\langle f, g\rangle_{L^{2}\left(\mathbf{R}_{+}, \Delta d x\right)}=\left\langle W_{+}(f), \tilde{W}_{+}^{-1}(g \Delta)\right\rangle_{L^{2}(\mathbf{R}, d x)}
$$

(cf. (32) for $\mathbf{R}_{+}$) and equivalently,

$$
\tilde{g}(\lambda+i \rho) C(-\lambda-i \rho)^{-1}=c\left(\tilde{W}_{+}^{-1}(g \Delta)\right)^{\sim}(-\lambda),
$$

where the symbol " $\sim$ " in the left (resp. right) hand side denotes the complex Fourier-Jacobi (resp. the Euclidean Fourier) transform. Hence the following definition of a dual space of $W_{-}\left(H^{1}(\mathbf{R})\right)$ is quite natural.

Definition 8.7. We define

$$
W_{-}\left(H^{1}(\mathbf{R})\right)^{*}=\left\{f \in L_{\mathrm{loc}}^{1}(G / / K) ; \tilde{W}_{+}^{-1}(f \Delta) \in B M O(\mathbf{R})\right\}
$$

and $\|f\|_{W_{-}\left(H^{1}(\mathbf{R})\right)^{*}}=\left\|\tilde{W}_{+}^{-1}(f \Delta)\right\|_{B M O(\mathbf{R})}$.

In this definition $f \in W_{-}\left(H^{1}(\mathbf{R})\right)^{*}$ is required to be $W_{+}$-smooth.

Proposition 8.8. Let notation be as above.

(1) For $f \in W_{-}\left(H^{1}(\mathbf{R})\right)$ and $h \in W_{-}\left(H^{1}(\mathbf{R})\right)^{*}$,

$$
\left|\int_{G} f(g) h(g) d g\right| \leq c\|f\|_{W_{-}\left(H^{1}(\mathbf{R})\right)}\|h\|_{W_{-}\left(H^{1}(\mathbf{R})\right)^{*}}
$$

(2) All K-bi-invariant $W_{+}$-smooth bounded functions belong to $W_{-}\left(H^{1}(\mathbf{R})\right)^{*}$.

Proof. (1) is clear from the above observation and the dual inequality between $H^{1}(\mathbf{R})$ and $B M O(\mathbf{R})$ (see $[15$, p. 146]). As for (2), let $f$ be a $K$-bi-invariant $\tilde{W}_{+}$-smooth bounded function on $G$. Then $\tilde{W}_{+}^{-1}(f \Delta) \in L^{\infty}(\mathbf{R})$ by [10, Lemma 3.3]. Since $L^{\infty}(\mathbf{R}) \subset B M O(\mathbf{R}), f$ belongs to $W_{-}\left(H^{1}(\mathbf{R})\right)^{*}$.

D. Real Hardy spaces: In Definition 7.3 we introduced $W_{-}\left(H_{\infty, 0}^{1, \epsilon}(\mathbf{R})\right)$ as a subspace of $W_{-}\left(H^{1}(\mathbf{R})\right)$. Here we shall define a subspace $W_{-}\left(\mathrm{H}_{\infty, 0}^{1, \epsilon}(\mathbf{R})\right)$ of $W_{-}\left(H_{\infty, 0}^{1, \epsilon}(\mathbf{R})\right)$, which corresponds to $H_{\infty, 0}^{1, \epsilon}(G / / K)$. Let $A$ be a centered $(1, \infty, 0, \epsilon)$ atom on $\mathbf{R}$ and suppose that $A$ is even. For $x_{0} \geq 0$, we define

$$
A_{x_{0}}(x)=\tilde{W}_{+}(A(x-s))\left(x_{0}\right) \Delta\left(x_{0}\right)^{-1}, \quad x>0,
$$


where $\tilde{W}_{+}$acts on $s$ and $\tilde{W}_{+}(F)\left(x_{0}\right) \Delta\left(x_{0}\right)^{-1}=F$ if $x_{0}=0$. Then, it follows from $\left[10\right.$, Lemma 3.3] that $A_{x_{0}}$ is also a $(1, \infty, 0, \epsilon)$-atom on $\mathbf{R}$. We introduce a modified atomic Hardy space $\mathrm{H}_{\infty, 0}^{1, \epsilon}(\mathbf{R})$ on $\mathbf{R}$ as the space of all $\sum_{i} \lambda_{i}\left(A_{i, x_{i}}(x)+A_{i, x_{i}}(-x)\right)$ such that $\sum\left|\lambda_{i}\right|<\infty, x_{i} \geq 0$ and each $A_{i}$ is an even centered $(1, \infty, 0, \epsilon)$-atom on R. Moreover, we define $\mathrm{H}_{\infty, 0}^{1, \epsilon}(\mathbf{R})_{\alpha}$ if each $A_{i}$ furthermore satisfies

$$
\left\|W_{-s_{\alpha}}^{\mathbf{R}}(A)\right\|_{\infty} \leq r^{-1}(1+r)^{-\epsilon} .
$$

Since each $A_{i, x_{i}}$ is a $(1, \infty, 0, \epsilon)$-atom on $\mathbf{R}$, it follows that

$$
\mathrm{H}_{\infty, 0}^{1, \epsilon}(\mathbf{R})_{\alpha} \subset \mathrm{H}_{\infty, 0}^{1, \epsilon}(\mathbf{R}) \subset H_{\infty, 0}^{1, \epsilon}(\mathbf{R}) \subset H^{1}(\mathbf{R})
$$

Definition 8.9. We define

$$
W_{-}\left(\mathrm{H}_{\infty, 0}^{1, \epsilon}(\mathbf{R})\right)=\left\{f \in L_{\mathrm{loc}}^{1}(G / / K) ; W_{+}(f) \in \mathrm{H}_{\infty, 0}^{1, \epsilon}(\mathbf{R})\right\}
$$

and give their norms by $\left\|W_{+}(f)\right\|_{H^{1}(\mathbf{R})}$. Similarly, we define $W_{-}\left(\mathbf{H}_{\infty, 0}^{1, \epsilon}(\mathbf{R})_{\alpha}\right)$.

Proposition 8.10. Let notation be as above. Then

$$
\mathrm{H}_{\infty, 0}^{1,1}(G / / K) \subset W_{-}\left(\mathrm{H}_{\infty, 0}^{1}(\mathbf{R})\right)
$$

Proof. Let $a$ be a centered $(1, \infty, 0,1)$-atom on $G$ supported on $B(r)$. We put $A=W_{+}(a)$. Since $A$ is also supported on $B(r)$ (see Lemma 5.11), it follows that

$$
\begin{aligned}
\left|W_{+}(A)(x)\right| & \leq c e^{2 \rho x}(\operatorname{th} r)^{s_{\alpha}}\|a\|_{\infty} \\
& \leq c e^{2 \rho r}(\operatorname{th} r)^{s_{\alpha}}|B(r)|^{-1}(1+r)^{-1} \leq c r^{-1} .
\end{aligned}
$$

Moreover, $A$ satisifies the moment condition, because $A^{\sim}(0)=\hat{a}(i \rho)=0$. Therefore, $A$ is a $(1, \infty, 0)$-atom on $\mathbf{R}$. We here note the following.

LemMA 8.11. $W_{+}\left(a_{x_{0}}^{b}\right)=W_{+}(a)_{x_{0}}$ on $\mathbf{R}_{+}$, that is,

$$
W_{+}\left(a_{x_{0}}^{b}\right)(x)=\tilde{W}_{+}\left(W_{+}(a)(y-s)\right)\left(x_{0}\right) \Delta\left(x_{0}\right)^{-1}, \quad x>0,
$$

where $\tilde{W}_{+}$acts on $s$.

Proof. Let $B=W_{+}\left(a_{x_{0}}^{b}\right)$. Since $e^{-\rho y} A(y), e^{-\rho y} B(y)$ are even, we see that

$$
\begin{aligned}
\left(e^{-\rho y} B(y)\right)^{\sim}(\lambda) & =\hat{a}_{x_{0}}(\lambda) \\
& =\hat{a}(\lambda) \varphi_{\lambda}\left(x_{0}\right) \\
& =\left(e^{-\rho y} A(y)\right)^{\sim}(\lambda) \tilde{W}_{+}\left(e^{-\rho s} \cos \lambda s\right)\left(x_{0}\right) \Delta\left(x_{0}\right)^{-1} \\
& =\tilde{W}_{+}\left(\left(e^{-\rho y} A(y-s)\right)^{\sim}(\lambda)\right)\left(x_{0}\right) \Delta\left(x_{0}\right)^{-1} .
\end{aligned}
$$


Hence, $e^{-\rho y} B(y)=\tilde{W}_{+}\left(e^{-\rho y} A(y-s)\right)\left(x_{0}\right) \Delta\left(x_{0}\right)^{-1}$ and the desired relation follows.

Lemma 8.11 and the fact shown before the lemma yield that each $a_{x_{0}}^{b}$ belongs to $W_{-}\left(\mathrm{H}_{\infty, 0}^{1}(\mathbf{R})\right)$ and the norm is bounded by a constant independent of $a, x_{0}$. Hence, the desired result follows.

Proposition 8.12. Let notation be as above. Then

$$
W_{-}\left(\mathrm{H}_{\infty, 0}^{1,1}(\mathbf{R})_{\alpha}\right) \subset \mathrm{H}_{\infty, 0}^{1}(G / / K) .
$$

Proof. Let $A$ be an even centered $(1, \infty, 0,1)$-atom on $\mathbf{R}$ supported on $B(r)$ and moreover, satisfying (94). Let $a=W_{-}(A)$. Then Lemma 8.11 implies that $A_{x_{0}}(x)+A_{x_{0}}(-x)=W_{+}\left(a_{x_{0}}^{b}\right)(x)$. Therefore, it is enough to show that $a$ has a decomposition $a=\sum_{i} \lambda_{i} a_{i}$ such that $\sum_{i}\left|\lambda_{i}\right| \leq c$ and each $a_{i}$ is a centered $(1, \infty, 0)$-atom on $G$, where $c$ is independent of $A$. Since $A$ is a $(1, \infty, 0,1)$-atom supported on $B(r)$ and satisfying (94), as in the proof of Proposition 6.2, it follows that

$$
|a(x)| \leq c \Delta(x)^{-1} r^{-1}(1+r)^{-1}
$$

We put

$$
a_{+}(x)=c \Delta(x)^{-1} r^{-1} \chi_{[0, r]}(x), \quad x>0 .
$$

Then $|a(x)| \leq a_{+}(x)$ and $a_{+}$is a non-increasing function on $\mathbf{R}_{+}$with finite $L^{1}$ norm. Since $a$ is supported on $B(r),|B(s)|^{-1} \int_{s}^{\infty} a_{0}(x) \Delta(x) d x$ is also supported on $B(r)$. For $0<s<r$, it follows from the moment condition that

$$
\frac{1}{|B(s)|} \int_{s}^{\infty} a_{0}(x) \Delta(x) d x=\frac{1}{|B(s)|} \int_{0}^{s} a_{0}(x) \Delta(x) d x \leq c|B(s)|^{-1} s r^{-1}(1+r)^{-1}
$$

Since $|B(s)| \sim \Delta(s) s$ if $s \leq 1$ and $|B(s)| \sim \Delta(s)$ if $s \geq 1$, it follows that

$$
\frac{1}{|B(s)|} \int_{s}^{\infty} a_{0}(x) \Delta(x) d x \leq a_{+}(s)
$$

This means that $a_{+}$is an $L^{1}$ non-increasing denominator of $a$ satisfying (95). Then [7, Theorem 4.5] yields that $a$ has a centered $(1, \infty, 0)$-atomic decomposition $a=$ $\sum_{i} \lambda_{i} a_{i}$ on $G$ such that $\sum_{i}\left|\lambda_{i}\right| \leq\left\|a_{+}\right\|_{L^{1}(\Delta d x)}$.

Main relationship among real Hardy spaces defined in this paper (see Definitions $4.1,4.2,5.2,5.3,5.8,7.2$, and 8.9$)$ is summarized in the following diagram, where we abbreviate " $G / / K$ " as $G$. The relation between $H^{1}(G / / K)$ and $H_{\infty, 0}^{1}(G / / K)$ is still open. 


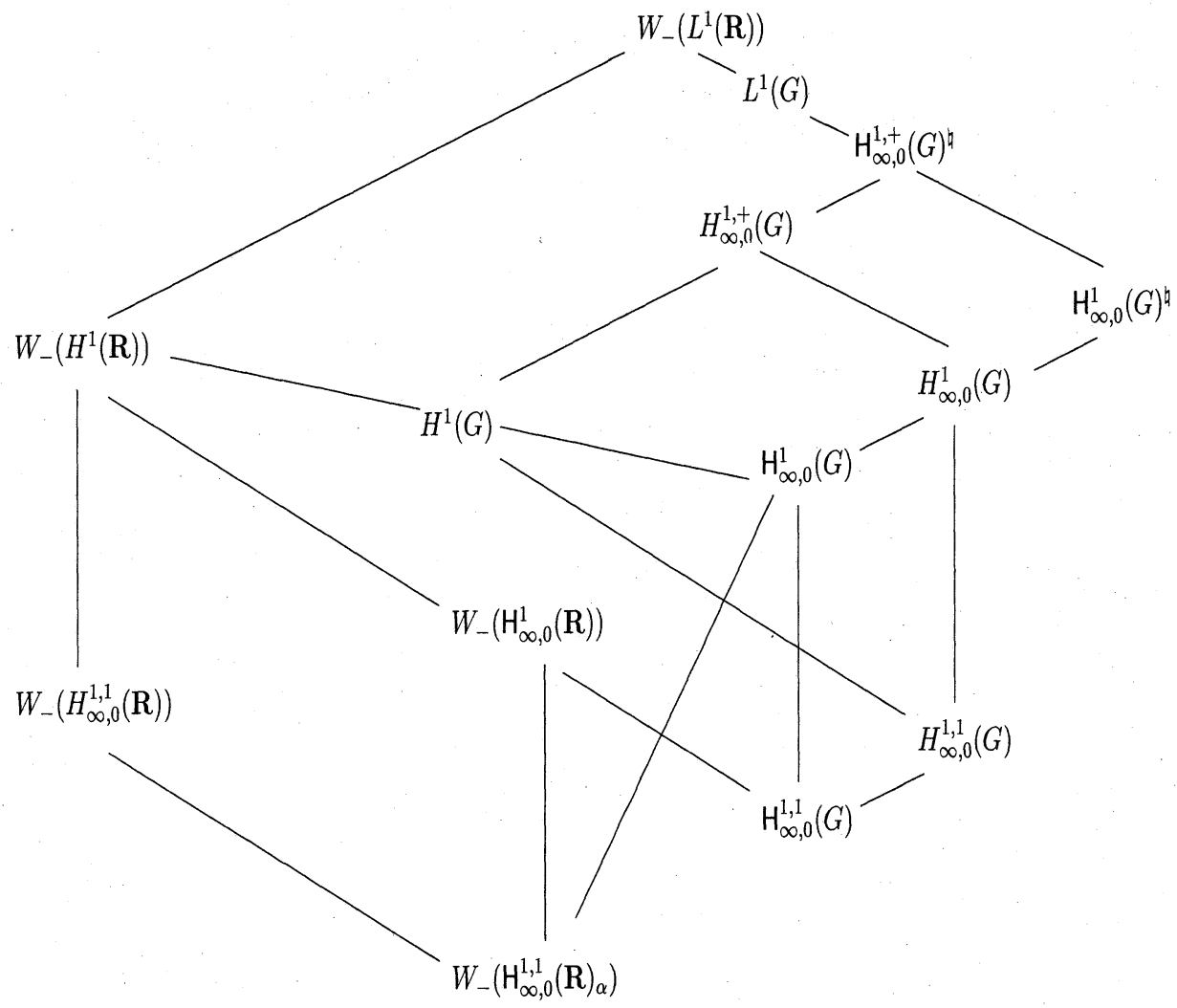

Figure 1. Real Hardy Spaces

ACKnowledgments. This work was accomplished while I was visiting l'Institut Élie Cartan, Université Henri Poincaré Nancy I in 1998 and 2003. I am grateful to Département de Mathématiques for hospitality, and to J-Ph., Anker, who is now at Université d'Orléans, and to Jean-Louis Clerc for stimulating and helpful discussions.

\section{References}

[1] J.-Ph. Anker, Sharp estimates for some functions of the Laplacian on noncompact symmetric spaces, Duke Math. J., 65 (1992), 257-297.

[2] R.R. Coifman and G. Weiss, Extensions of Hardy spaces and their use in analysis, Bull. Amer. Math. Soc., 83 (1977), 569-645.

[3] M. Flensted-Jensen, Paley-Wiener type theorems for a differential operator connected with symmetric spaces, Ark. Mat., 10 (1972), 143-162. 
[4] M. Flensted-Jensen and T. Koornwinder, The convolution structure and Jacobi transform expansions, Ark. Mat., 11 (1973), 245-262.

[5] M. Flensted-Jensen and T. Koornwinder, Positive definite spherical functions on a noncompact, symmetric space, Lecture Notes in Math., 739, pp. 249-282, Springer, 1979.

[6] G.B. Folland and E.M. Stein, Hardy Spaces on Homogeneous Groups, Mathematical Notes 28, Princeton University Press, New Jersey, 1982.

[ 7 ] T. Kawazoe, Atomic Hardy spaces on semisimple Lie groups, Japan. J. Math., 11 (1985), 293-343.

[ 8 ] T. Kawazoe, $L^{1}$ estimates for maximal functions and Riesz transform on real rank 1 semisimple Lie groups, J. Funct. Anal., 157 (1998), 327-527.

[9] T. Kawazoe, Hardy spaces and maximal operators on real rank 1 semisimple Lie groups I, Tohoku Math. J., 52 (2000), 1-18.

[10] T. Kawazoe and J. Liu, Fractional calculus and analytic continuation of the complex FourierJacobi transform, Tokyo J. Math., 27 (2004), 187-207.

[11] T. Koornwinder, A new proof of a Paley-Wiener type theorem for the Jacobi transform, Ark. Mat., 13 (1975), 145-159.

[12] R.H. Latter, A decomposition of $H^{p}\left(\mathbf{R}^{n}\right)$ in terms of atoms, Studia Math., 62 (1978), 92-101.

[13] B. Rubin, Fractional Integrals and Potentials, Pitman Monographs and Surveys in Pure and Applied Mathematics, 82, Addison-Wesley, Longman, London, 1996.

[14] E.M. Stein, Topics in Harmonic Analysis. Related to the Littlewood-Paley Theory, Annals of Mathematics Studies, 63, Princeton University Press, New Jersey, 1970.

[15] E.M. Stein, Harmonic Analysis, real-variable methods, orthogonality, and oscillatory integrals, Princeton Mathematical Series, 43, Princeton University Press, New Jersey, 1993.

[16] A. Torchinsky, Real-variable Methods in Harmonic Analysis, Pure and Applied Mathematics, 123, Academic Press, Orlando, Florida, 1986.

[17] G. Warner, Harmonic Analysis on Semi-Simple Lie Groups II, Springer-Verlag, New York, 1972.

Department of Mathematics

Keio University at Fujisawa

Endo, FujISAWA, KANAGAWA, 252-8520, JAPAN

E-mail: kawazoe@sfc.keio.ac.jp 